volume 2 ISSUE 1

2014 Giugno

Editor in Chief Roberto Nardi

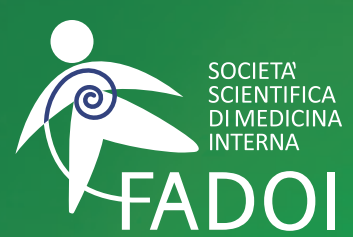

FEDERAZIONE

DELLE ASSOCIAZIONI

DEI DIRIGENTI

OSPEDALIERI

INTERNISTI
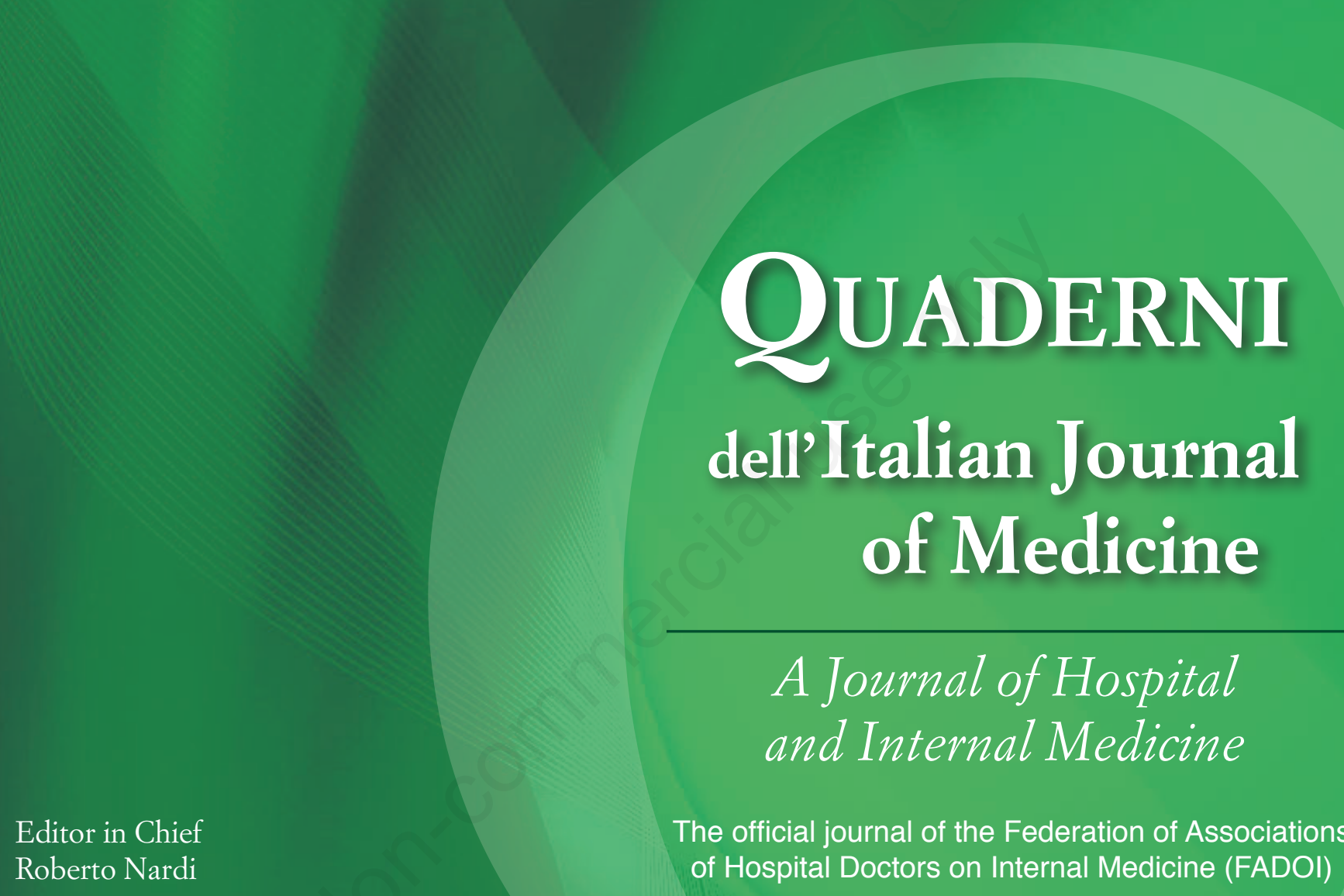

A Joumal of Hospital

and Internal Medicine

The official journal of the Federation of Associations of Hospital Doctors on Internal Medicine (FADOI)

\title{
La Medicina Interna ospedaliera: tra complessità ed intensità assistenziale verso una medicina sostenibile
}

Guest Editors: M. Campanini, A. Fontanella, G. Nozzoli, A. Mazzone, R. Nardi 
Mauro Campanini, Novara, Italy

\section{PRESIDENTE ELETTO}

Andrea Fontanella, Napoli, Italy

\section{PAST PRESIDENT}

Carlo Nozzoli, Firenze, Italy

\section{SEGRETARIO}

Giuseppe Augello, Canicattì (AG), Italy

\section{TESORIERE}

Dario Manfellotto, Roma, Italy

DIRETTORE RIVISTA

ITALIAN JOURNAL OF MEDICINE

Roberto Nardi, Bologna, Italy
RESPONSABILE RAPPORTI SOCIETÀ SCIENTIFICHE E ISTITUZIONI

Antonino Mazzone, Legnano (MI), Italy

RESPONSABILE SISTEMA GESTIONE QUALITÀ

Franco Berti, Roma, Italy

RESPONSABILE SITO NAZIONALE

Francesco Cipollini, Ascoli Piceno, Italy

DELEGATO FADOI ITALIAN STROKE ORGANIZATION

Michele Stornello, Siracusa, Italy

COMMISSIONE FADOI GIOVANI

Paola Gnerre, Savona, Italy

\section{Fondazione FADOI - Organigramma}

PRESIDENTE

Giorgio Vescovo, Vicenza, Italy

\section{COORDINATORE}

Domenico Panuccio, Bologna, Italy

\section{DIRETTORE SCIENTIFICO}

Gualberto Gussoni, Milano, Italy

DIRETTORE DIPARTIMENTO PER LA FORMAZIONE ED AGGIORNAMENTO

Mauro Silingardi, Guastalla (RE), Italy
DIRETTORE DIPARTIMENTO PER LA RICERCA CLINICA

Giancarlo Agnelli, Perugia, Italy

\section{CONSIGLIO DI AMMINISTRAZIONE}

Consigliere

Consigliere

Consigliere

Consigliere

Consigliere

Consigliere

Consigliere

Consigliere

Consigliere
Paolo Arullani, Roma, Italy

Davide Croce, Castellanza (VA), Italy

Francesco D'Amore, Roma, Italy

Salvatore Di Rosa, Palermo, Italy

Sandro Fontana, Biella, Italy

Ranieri Guerra, Roma, Italy

Ido Iori, Reggio Emilia, Italy

Giovanni Mathieu, Pinerolo (TO), Italy

Cecilia Politi, Isernia, Italy 


\section{QUADERNI - Italian Journal of Medicine}

\section{INDICE}

INTRODUZIONE

La Medicina Interna ospedaliera: tra complessità ed intensità assistenziale verso una medicina sostenibile

\section{RASSEGNE}

La complessità dei pazienti ricoverati nei reparti ospedalieri

di Medicina Interna: di che cosa stiamo parlando?

R. Nardi, D. Borioni, F. Berti, A. Greco, G. Scanelli, P. Leandri, M. Reta,

M. Mazzetti, A. Pasquale, G. Belmonte, M. Magnani, S. Frasson, C. Baldo,

G. Gussoni, G. Vescovo, M. La Regina, M. Campanini, I. Iori, G. Mathieu,

A. Mazzone, C. Nozzoli, A. Fontanella

Strumenti di valutazione della complessità nel paziente internistico: un percorso ancora da tracciare

S. Frasson, R. Nardi

La stratificazione per severità dei pazienti ricoverati in Medicina Interna: un lavoro ancora incompiuto.

Valutazione clinica e non strumenti surrogati

G. Chesi, R. Nardi

Nella Medicina Interna ospedaliera vogliamo un sistema sanitario pubblico sostenibile: considerazioni e proposte di FADOI

per ridurre le spese inappropriate $\ldots \ldots \ldots \ldots \ldots \ldots \ldots \ldots$

R. Nardi, D. Borioni, A. Pasquale, G. Chesi, F. Berti, L.M. Fabbri, I. Iori,

G. Mathieu, A. Mazzone, M. Campanini, C. Nozzoli, A. Fontanella

Il medico ed il suo paziente: fare giusto, non troppo nè troppo poco . .

F. D'Amore

Il decalogo ANÌMO per un'assistenza Slow Medicine: le raccomandazioni generali degli infermieri di Medicina Interna per un'assistenza sobria, rispettosa e giusta. ................ G. Bordin, M. Bonfanti, F. Calabria, R. Cossu, C. Cozzi, T. Esposito, C. Ganzini, E. Gragnoli, R. Leotta, M. Lince, L. Lucci, G. Manfroni, M. Martini, G. Pentella, N. Postal, R. Rapetti, O. Teti, G. Zocchi, A. Zuccone, C. Gatta

Come organizzare l'Area Medica per affrontare le nuove sfide della complessità clinica ed assistenziale.

I modelli organizzativi per l'assistenza territoriale primaria nel Servizio Sanitario Nazionale:

evidenze ed intervento specialistico $\ldots \ldots \ldots \ldots \ldots \ldots \ldots \ldots \ldots$

D. Croce, A. Mazzone

La Medicina Interna ospedaliera: tra complessità ed intensità assistenziale verso una medicina sostenibile. 
A

\title{
FRANCESCO CAVAZZUTI
}

\author{
maxima debetur puero reverentia, ${ }^{1}$ \\ sed etiam seni
}

${ }^{1}$ (Giovenale, Satire, XIV, 47)

Maestro di tutti noi, professore ma anche grande amico, ci hai insegnato la sobrietà, per una medicina etica, rispettosa della persona anziana.

Roberto Nardi 


\title{
La Medicina Interna ospedaliera: tra complessità ed intensità assistenziale verso una medicina sostenibile
}

\author{
Mauro Campanini \\ Medicina Interna 2 - Dipartimento Medico, AO Maggiore della Carità, Novara, Italia
}

L'invecchiamento progressivo della popolazione in Europa ed in Italia ha determinato la comparsa di nuovi problemi clinici ed assistenziali con un incremento dei costi in un sistema però a risorse limitate. È comparso un nuovo modello di paziente, ove spesso non è la singola patologia a condizionare la prognosi, ma l'insieme di più fattori che, attraverso la multimorbidità, determinano la complessità del paziente stesso. Pertanto al centro del processo assistenziale è lo stato di complessità che inevitabilmente condiziona le scelte cliniche e determina $\mathrm{i}$ costi della sua gestione. Questo modello di paziente, che sempre di più siamo chiamati a gestire, rappresenta una novità in ambito sanitario ed accademico se si pensa che non è considerato nel percorso di insegnamento della Laurea in Medicina e Chirurgia e nelle Scuole di specializzazione di area medica ove il percorso didattico è dedicato alla singola patologia del singolo apparato e non al problema dell'associazione di più patologie e di quello che questo comporta. Nella pratica quotidiana la multimorbidità e la complessità clinico-assistenziale del singolo paziente costituiscono vere e proprie sfide che dobbiamo affrontare spesso senza supporti scientifici, guidati solamente dalla nostra cultura, dal senso clinico e dall'esperienza. Questa nuova situazione epidemiologica vede la Medicina Interna quale naturale gestore di questo cambiamento clinico epocale. Ma anche noi specialisti in Medicina Interna dobbiamo dedicare attenzione, tempo e ricerca per sviluppare un percorso che ci permetta di affrontare la complessità clinica in modo strutturato e razionale; in questo contesto è fondamentale riuscire ad individuare delle priorità nell'affrontare i problemi clinici sia acuti che

Corrispondente: Mauro Campanini, Medicina Interna 2 Dipartimento Medico, AO Maggiore della Carità, Novara, Italia.

E-mail: mauro.campanini@maggioreosp.novara.it

Articolo pubblicato secondo la Creative Commons Attribution NonCommercial 3.0 License (CC BY-NC 3.0).

CCopyright M. Campanini, 2014

Licensee PAGEPress, Italy

QUADERNI - Italian Journal of Medicine 2014; 2:1-2 cronici e le scelte diagnostiche e terapeutiche che queste comportano.

La crescente complessità clinica impone, pertanto, anche scelte organizzative e gestionali innovative al fine di poter affrontare nel modo più idoneo possibile i problemi clinico-assistenziali dei nostri pazienti con l'obiettivo di offrire non solo la cura giusta ma assicurare che questa venga erogata anche nel tempo e nel posto giusto. Mi riferisco in particolare alla necessità per la Medicina Interna della costituzione di aree dedicate al paziente critico ove si affrontino i problemi acuti in modo adeguato sia per la tecnologia offerta che per un più elevato livello di assistenza medica ed infermieristica. In un Sistema Sanitario però che pone fra i suoi obiettivi la riduzione o perlomeno il contenimento dei costi, è solo attraverso un'analisi dei problemi ed una loro precisa conoscenza che sarà possibile intraprendere le scelte gestionali più idonee nell'ottica di un'ottimizzazione delle risorse. È opportuno pertanto ritornare ad una visione delle medicina che non consideri al centro del percorso di cura la malattia ma un processo olistico centrato sulla persona. Il nostro paziente è un paziente nuovo perché complesso. Pertanto il paziente non deve essere scomposto nelle singole patologie di cui è affetto ma, attraverso la posizione centrale della Medicina Interna, considerato nel suo insieme perché solo così può essere curato il malato e non la malattia. È chiaro che in questo percorso la Medicina Interna non può essere sola ma deve potersi avvalere di tutte le altre specialità mediche.

Compito di una Società Scientifica di Medicina Interna come la FADOI, è pertanto quello di analizzare e studiare la complessità, cercando di misurare questo fenomeno, di stratificare i pazienti in funzione della loro gravità e di organizzare la propria attività in modo tale da fornire le migliori risposte cliniche ed assistenziali. Questo processo vede nel Dipartimento medico la sede più naturale per realizzare questi obiettivi. In questo ambito sarà poi fondamentale stabilire una serie di pratiche cliniche essenziali e di stabilire una priorità negli interventi, eliminando le ridondanze, per rendere sempre più sostenibile la nostra medicina.

È evidente che il percorso assistenziale del pa- 
ziente non possa esaurirsi solo nell'ambito del ricovero ospedaliero ma debba essere più complessivo $\mathrm{e}$ continuativo e considerare il territorio come la sede naturale della prosecuzione del percorso di cura. Si deve tendere ad una migliore e nuova integrazione ospedale territorio per il paziente complesso.
Abbiamo pertanto, per tutte queste ragioni, deciso di dedicare un volume monografico dei Quaderni dell'Italian Journal of Medicine, allo studio degli aspetti connessi con la gestione clinica ed assistenziale del paziente medico complesso nell'ottica però sempre di una Medicina sostenibile. 


\title{
La complessità dei pazienti ricoverati nei reparti ospedalieri di Medicina Interna: di che cosa stiamo parlando?
}

\author{
Roberto Nardi, ${ }^{1}$ Daniele Borioni, ${ }^{1}$ Franco Berti, ${ }^{2}$ Antonio Greco, ${ }^{3}$ Giovanni Scanelli, ${ }^{4}$ Paolo Leandri, ${ }^{1}$ \\ Massimo Reta, ${ }^{1}$ Magda Mazzetti, ${ }^{1}$ Angelo Pasquale, ${ }^{1}$ Gelorma Belmonte, ${ }^{1}$ Mirco Magnani, ${ }^{1}$ Stefania Frasson, ${ }^{5}$ \\ Concetta Baldo, ${ }^{5}$ Gualberto Gussoni, ${ }^{5}$ Giorgio Vescovo, ${ }^{6}$ Micaela La Regina, ${ }^{7}$ Mauro Campanini, ${ }^{8}$ Ido Iori, ${ }^{9}$ \\ Giovanni Mathieu, ${ }^{10}$ Antonino Mazzone, ${ }^{11}$ Carlo Nozzoli, ${ }^{12}$ Andrea Fontanella ${ }^{13}$
}

${ }^{1}$ Azienda USL di Bologna, Ospedale Maggiore, Medicina Interna, Bologna; ${ }^{2}$ Medicina Interna III, AO San Camillo-Forlanini, Roma; ${ }^{3}$ UO Geriatria, IRCCS Casa Sollievo della Sofferenza, San Giovanni Rotondo (FG); ${ }^{4}$ Medicina Interna Ospedaliera, AOU Ferrara Arcispedale Sant'Anna, Ferrara; ${ }^{5}$ Centro Studi FADOI, Milano; ${ }^{6}$ UOC Medicina Interna, Vicenza; ${ }^{7}$ Medicina Interna, POU del Levante Ligure, ASL 5, La Spezia; ${ }^{8}$ Medicina Interna II e d'Urgenza, Dipartimento Emergenza ed Accettazione, AO Maggiore della Carità, Novara; ${ }^{9}$ UOC Medicina Interna e Centro Emostasi e Trombosi, AO di Reggio Emilia, Arcispedale Santa Maria Nuova, Reggio Emilia; ${ }^{10}$ Past Director UOC di Medicina Interna, Ospedale E. Agnelli di Pinerolo (TO);

${ }^{11}$ Dipartimento di Area Medica, UOC di Medicina Interna, Ospedale Civile, Legnano (MI); ${ }^{12}$ Medicina Interna e d'Urgenza, AOU Careggi Firenze, Italy; ${ }^{13}$ Dipartimento di Medicina, Ospedale Madonna del Buon Consiglio Fatebenefratelli, Napoli, Italia

\section{RIASSUNTO}

I pazienti di Medicina Interna (MI) sono per lo più anziani, con diverse multimorbilità, di solito croniche. La complessità di questi pazienti implica un'intricata interazione fra due o più sistemi (ad esempio, il corpo e/o la psiche e la malattia, il contesto socio-economico, famigliare e ambientale, il coordinamento delle cure e delle terapie. Tutto ciò richiede una valutazione multidimensionale (VMD) dei problemi in causa. Nonostante i tentativi per migliorare la gestione delle patologie croniche e la disponibilità di diverse metodiche di $\mathrm{VMD}$, definire il paziente complesso non è a tutt'oggi un processo acquisito in maniera univoca. Il profilo complesso dei nostri pazienti può essere descritto solamente mediante strumenti progettati e validati ad hoc. Per questo la FADOI, Federazione delle Associazioni dei Dirigenti Ospedalieri Internisti, vuole assumere iniziative finalizzate a comprendere le diverse esigenze del paziente, analizzare i cluster di malattie e la possibile relazione tra loro nei pazienti ricoverati in Medicina Interna, per identificarne le caratteristiche. Esplorando la complessità dei nostri pazienti, evidenziando i loro bisogni reali, possiamo effettuare scelte appropriate per il loro trattamento. Abbiamo bisogno di perseguire l'approccio di valutazione più semplice possibile per i nostri pazienti complessi, con strumenti che possano essere utilizzati nella valutazione clinica e nella stratificazione prognostica, anche ai fini di una selezione gerarchica delle priorità e della selezione dei pazienti più vulnerabili. Riteniamo che siano necessarie ulteriori indagini e studi sui pazienti complessi ricoverati nei reparti di MI.

Corrispondente: Roberto Nardi, via C. Pavese 16/2, 40141 Bologna, Italia.

Tel.: +39.335.8291342. E-mail: nardidoc48@gmail.com

Parole chiave: comorbilità, multimorbilità, complessità, fragilità, valutazione multidimensionale, pazienti di Medicina Interna.

Ringraziamenti: si ringrazia la dott.ssa S. Nardi per la preziosa collaborazione fornita nella revisione italiana dell'articolo originale.

Modificato da: R. Nardi, F. Berti, A. Greco, et al. Complexity in hospital internal medicine departments: what are we talking about? Ital J Med 2013;7:142-55.

Articolo pubblicato secondo la Creative Commons Attribution NonCommercial 3.0 License (CC BY-NC 3.0).

CCopyright R. Nardi et al., 2014

Licensee PAGEPress, Italy

QUADERNI - Italian Journal of Medicine 2014; 2:3-13

\section{Introduzione}

La medicina moderna soffre di due principali paradossi: ${ }^{1,2}$ i) stiamo ancora praticando una medicina per ammalati acuti in un mondo di malattie croniche; ${ }^{a}$ ii) i modelli medici tradizionali si fondano su interazioni lineari, restrittive e semplificate degli elementi in causa. La realtà è ben diversa: i pazienti presentano

a Intendiamo per malattia acuta un processo morboso ad esordio improvviso, di solito di breve durata, rapidamente progressiva, che necessita di cure tempestive e/o urgenti, con evoluzione nella maggioranza dei casi in guarigione, in relazione sia al trattamento, ma anche, indipendentemente dall'intervento di un farmaco o provvedimento terapeutico specifico, per le naturali difese dell'organismo. Una malattia cronica è intesa come una malattia prolungata, curabile ma non guaribile, che si sviluppa lentamente e persiste nel tempo, spesso per il resto della vita dell'individuo. Le malattie croniche durano per tutta una vita, si accumulano con l'età, sono in genere progressive, condizionano in maniera rilevante la qualità della vita. 
elementi di complessità di non semplice gestione. ${ }^{3} \mathrm{Un}$ sistema di erogazione delle cure che non tenga conto delle differenze tra pazienti acuti e cronici e delle peculiarità di questi ultimi, può comportare diverse conseguenze, sintetizzate nella Tabella 1.

I pazienti di Medicina Interna (MI) sono principalmente anziani o molto anziani, hanno solitamente multiple comorbidità, comprendenti patologie acute, ma più frequentemente croniche, che limitano il loro grado di autonomia. ${ }^{4,5}$ In questi pazienti è importante ricercare la comorbidità, ma questa non è in grado, di per sé, di riflettere la complessità, né tantomeno, la fragilità ad essa frequentemente correlata. ${ }^{6}$ È importante sottolineare le differenze tra i concetti di multi-morbilità e complessità, per evidenziare le implicazioni connesse e le possibili aree di incertezza, per ricercare strumenti per la loro misurazione e definire le responsabilità di internisti nella valutazione e nella gestione dei pazienti ricoverati nei loro reparti. Non esiste nessun indicatore amministrativo o indice di comorbilità, che, pur se validato in pazienti anziani, sia in grado di definire esaustivamente la complessità dei nostri pazienti. Il lavoro quotidiano degli internisti, proprio per la molteplicità dei problemi posti, diventa una vera e propria sfida, che richiede competenze specifiche (Tabella 2) (Figure 1-3). ${ }^{7,8}$

\section{Comorbilità e multimorbilità: come definire la presenza di malattie multiple nello stesso paziente?}

La presenza di malattie multiple nello stesso individuo costituisce oggi una sfida per qualsiasi medico che si occupi di pazienti anziani. ${ }^{28-31}$ Comorbilità e multimorbilità vanno distinte dalle complicazioni, acquisite durante il ricovero in ospedale..$^{32}$ Definire la comorbilità in un paziente non è semplice. ${ }^{33-35}$ Nello studio REPOSI è stato impiegato il termine cluster di più malattie, per indicare la compresenza di due o più malattie croniche specifiche. ${ }^{11}$ Anche recentemente è stata ribadita la necessità di disporre di una definizione omnicomprensiva di mutimorbilità, particolarmente nel long term care, utile anche ai medici di famiglia. ${ }^{36}$
- Saper gestire condizioni cliniche in cui coesistono multiple malattie e situazioni complesse

- Guardare non solo agli organi ed apparati compromessi dalle patologie, ma anche alle preferenze ed agli obiettivi dei pazienti

- Contenere laddove possibile i trattamenti polifarmacologici

- Utilizzare in modo efficiente ed appropriato i servizi ospedalieri, anche valutando (quando realisticamente praticabili) alternative alla ospedalizzazione in caso di comparsa di patologie acute o riacutizzazione di malattie croniche

- Coordinare al meglio le transizioni tra i vari setting assistenziali

- Saper realizzare e partecipare a studi osservazionali e di ricerca traslazionale

Figura 1. Le competenze di base per saper curare i pazienti più anziani. Fonte: Leipzig et al., 2012. ${ }^{8}$

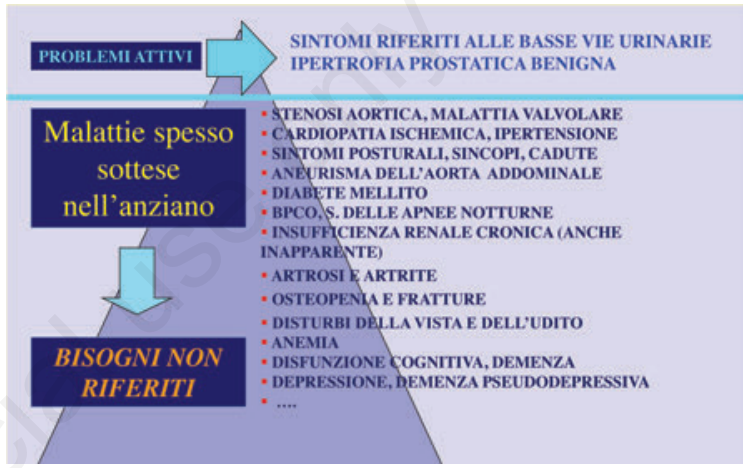

Figura 2. Malattie ad iceberg nell'anziano.

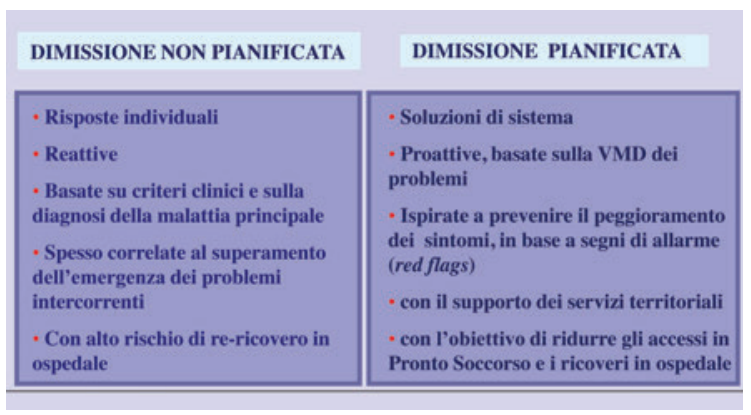

Figura 3. La pianificazione della dimissione: un confronto. VMD, valutazione multi-dimensionale.

Tabella 1. Possibili disfunzioni correlate alla gestione delle malattie croniche.

Troppi medici e sub-specialisti coinvolti nella gestione dello stesso paziente

Conseguente possibilità di consigli contrastanti

Scarsa educazione del paziente, carenza di informazioni e counseling al paziente, famigliari e caregivers

Sottostima nella diagnosi delle principali malattie croniche

Sottovalutazione dei sintomi/segni di peggioramento o riesacerbazione di una malattia cronica e incapacità nel riconoscerne i fattori destabilizzanti/precipitanti

Sovrastima delle malattie co-esistenti (a volte enfatizzate da sub-specialisti d'organo o apparato), il cui trattamento non è in grado di modificare la storia naturale della malattia di base principale

Insufficiente monitoraggio del paziente e follow up inadeguato

Scarso coordinamento delle cure, duplicazione di alcuni servizi, omissione di prestazioni necessarie

Errori medici, scarsa aderenza al trattamento, reazioni avverse da farmaci 
Tabella 2. Attributi e caratteristiche dei pazienti nei reparti di Medicina Interna: glossario.

\begin{tabular}{ll}
\hline Attributi & Caratteristiche e significato \\
\hline Comorbilità e multimorbilità & La maggior parte delle persone ricoverate nei reparti di MI hanno più di una \\
& condizione, con diverse sfide per la loro gestione \\
Vanno differenziati i concetti di comorbilità e multimorbilità e l'impatto & sulla salute del paziente delle malattie, in relazione alla loro severità \\
& (Tabella 5)
\end{tabular}

Malattie ad iceberg, bisogni non segnalati

Le persone anziane tendono a non segnalare e/o a sottovalutare i sintomi ed $\mathrm{i}$ problemi sottesi, per paura delle conseguenze e del rischio di ospedalizzazione. Nella valutazione globale del paziente, a fronte di espliciti problemi attivi, possiamo trovare malattie e condizioni non palesi, né dichiarate in anamnesi o non immediatamente riconoscibili, ma di per sé in grado di modificare il naturale decorso clinico e la prognosi (Figura 2)

\begin{tabular}{ll}
\hline Severità delle malattie & Riferita al grado di insufficienza d'organo o apparato \\
\hline Rischio di mortalità e cure di fine-vita & Riferita alla probabilità di morire \\
& Nei nostri pazienti il problema delle cure di fine-vita si pone spesso \\
Il problema di definire quale sia la migliore assistenza, non riguarda sola- \\
mente gli ultimi giorni o ore di vita, ma più in generale, quando una malattia \\
terminale sta diventando avanzata, progressiva e incurabile
\end{tabular}

Prognosi $\quad$ Riferita al probabile esito di una malattia, compresa la probabilità di miglioramento o di peggioramento della malattia, la sua probabile durata, la possibilità di recidive e la speranza di vita

Difficoltà di trattamento

Riferita ai possibili problemi di gestione del paziente da parte dei professionisti sanitari che se ne devono occupare

Tali problemi di gestione sono spesso correlati a malattie che si presentano con un quadro clinico incerto, che necessitano di procedure sofisticate e tecnicamente avanzate o che richiedono un attento monitoraggio e supervisione

Eventi avversi in ospedale ${ }^{9-11}$

Esempi di eventi avversi: cadute, infezioni nosocomiali, sepsi, reazioni da farmaci, sindromi da immobilizzazione a letto, ulcere da pressione, ecc, con possibili conseguenze dopo il ricovero come ad esempio: prolungata degenza in ospedale, eventi a cascata, la necessità di ulteriori interventi da parte degli infermieri e/o dei medici, perdita dell'autonomia, ospedalizzazioni ripetute o obitus.

\begin{tabular}{|c|c|}
\hline Errori contestuali e incapacità a trovare la cura adatta ${ }^{12}$ & $\begin{array}{l}\text { Un errore contestuale si verifica quando un medico tralascia alcuni elementi } \\
\text { essenziali riferiti al paziente, che sono indispensabili per pianificare la cura } \\
\text { appropriata } \\
\text { Una scarsa attenzione alle informazioni sulla situazione ambientale, eco- } \\
\text { nomica, famigliare, relative ai caregivers, al livello di autosufficienza e } \\
\text { alle esigenze di trasporto di un paziente può portare ad errori contestuali, } \\
\text { solitamente non esplicitamente evidenziati negli audit e nelle valutazione } \\
\text { di performance }\end{array}$ \\
\hline Necessità di un intervento & $\begin{array}{l}\text { Riguarda le conseguenze di una mancata cura tempestiva sulla gravità della } \\
\text { malattia }\end{array}$ \\
\hline (In)stabilità clinica & $\begin{array}{l}\text { La stabilità si riferisce alla capacità di un paziente di mantenere un equilibrio } \\
\text { di stato stazionario, compatibile con le normali funzioni fisiologiche }{ }^{14} \text { È le- } \\
\text { gata alla normalità o meno di funzioni vitali (ABC: vie respiratorie, respira- } \\
\text { zione, circolazione), pressione arteriosa, temperatura corporea, frequenza } \\
\text { cardiaca e respiratoria, alterato stato di coscienza } \\
\text { Con la valutazione di cinque semplici parametri fisiologici (pressione arte- } \\
\text { riosa sistolica, frequenza cardiaca, frequenza respiratoria, temperatura cor- } \\
\text { porea e livello di coscienza) mediante il MEWS (modified early warning } \\
\text { system) è possibile esprimere un giudizio prognostico sull'esito del ricovero } \\
\text { ospedaliero }{ }^{15} \\
\text { Tale metodo è comunque ancora oggi oggetto di discussione, con proposi- } \\
\text { zione di nuovi strumenti per valutare la stabilità del paziente, anche se la va- } \\
\text { lutazione clinica da parte del medico è irrinunciabile }\end{array}$ \\
\hline La disponibilità delle risorse & $\begin{array}{l}\text { Riferita alle risorse (personali, psicologiche, spirituali, sociali, tecnico-pro-- } \\
\text { fessionali e finanziarie) disponibili per il paziente, la famiglia e la comunità }\end{array}$ \\
\hline L'intensità delle risorse & $\begin{array}{l}\text { Si riferisce al volume relativo di professionisti (infermieri, medici, altri), ai } \\
\text { servizi di diagnosi e terapia, alle modalità di monitoraggio e alla disponibilità } \\
\text { di letti utilizzati nella gestione di una particolare malattia per singolo pa- } \\
\text { ziente, tenendo conto della quantità e della qualità di assistenza fornita }{ }^{17}\end{array}$ \\
\hline
\end{tabular}

Continua nella pagina seguente 
Tabella 2. Segue dalla pagina precedente

Complessità assistenziale infermieristica

Riguarda la gestione di alcuni aspetti di assistenza: la respirazione, la gestione del tubo oro-tracheale, della ventilazione non invasiva, eccetera; i mezzi di accesso venoso centrale o periferico; la nutrizione (enterale, parenterale) e l'idratazione; l'eliminazione urinaria ed intestinale, l'igiene personale, la postura ed il movimento del paziente; il riposo ed il sonno, la funzione cardiovascolare, l'ambiente sicuro, l'interazione, la comunicazione con il paziente i famigliari e i caregivers; la loro educazione sanitaria, le medicazioni avanzate ed i farmaci, le procedure diagnostico e terapeutiche, i dispositivi e devices, le modalità di monitoraggio.

I pazienti difficili

Alcuni pazienti (e, a volte, le loro famiglie) sono ostili, aggressivi, non collaboranti, sospettosi

In questi casi la gestione complessiva del paziente e del contesto famigliare può essere difficile e frustrante per il medico e l'infermiere ${ }^{18}$

Dimissioni difficili dall'ospedale ${ }^{19,20}$

Oltre alla natura e gravità delle malattie, le dimissioni difficili corrispondono a quelle situazioni riguardanti il paziente ricoverato in ospedale, in cui: siano riscontrabili situazioni umane, economiche, sociali, ambientali ed organizzative non facilmente risolvibili dal paziente e/o dalla sua famiglia, tali da indurre un blocco del letto in ospedale, ritardo della dimissione ospedaliera, prolungata degenza ospedaliera e un elevato rischio di riammissione precoce in ospedale

È necessario il coinvolgimento dei servizi territoriali

Re-ricoveri ospedalieri non programmati

Il numero di pazienti dimessi da un ospedale per acuti e riammessi in qualsiasi ospedale entro 30 giorni diviso per il numero totale di persone dimesse vive da reparti per acuti ${ }^{21}$

Tasso di riammissione dopo un determinato periodo di tempo

La gestione delle malattie croniche ${ }^{22}$

L'invecchiamento della popolazione richiede un sistema sanitario finalizzato al miglioramento di tutti i livelli dell'organizzazione

Troppi decisori non sono congruenti con un piano di assistenza coerente rispetto ad obiettivi terapeutici ed assistenziali selezionati*

Comunicazione e il coordinamento sono strumenti essenziali per la cura dei pazienti complessi

Il processo decisionale al letto del paziente

Qualsiasi decisione al letto del paziente (bed side) dovrebbe essere presa in base a un giudizio clinico adeguato, integrato da tutti gli strumenti appropriati disponibili

VMD globale

Definisce lo stato di salute di una persona anziana attraverso un'attenta analisi di differenti domini: capacità funzionale, biologico e clinico, psicologico, affettivo, sociale, ambientale, eccetera.

La VMD è utile nella definizione delle priorità di cura, in base al giudizio di fragilità o di robustezza (fitness) di ogni singolo paziente

Sulla VMD si fonda la decisione terapeutica o diagnostica, riguardo a misure di intervento invasive, conservative e/o palliative, a seconda dei casi ${ }^{23}$

Pianificazione della dimissione

Una gamma variegata e completa di servizi presenti nell'organizzazione sanitaria locale non è di per sé sufficiente

Non abbiamo bisogno di aumentare i servizi: dobbiamo essere in grado di guidare le persone nel sistema sanitario esistente, secondo un percorso ritagliato alle specifiche esigenze del paziente (Figure 3 e 4$)^{24}$

La gestione del dopo-dimissione

E' necessario identificare i pazienti a rischio di re-ricovero, per:

- effettuare un'adeguata educazione sanitaria (rivolta anche ai caregivers)

- approntare programmi assistenziali ad hoc

- pianificare fin dalla dimissione dall'ospedale i controlli necessari

La continuità delle cure

La continuità delle cure viene intesa come la sequenza logica degli interventi sanitari effettuati sul paziente anche dopo la dimissione dall'ospedale, congruente con obiettivi di salute condivisi nel sistema di assistenza ${ }^{25}$ Per il personale sanitario significa avere tutte le informazioni necessarie sul paziente nel setting di cura (continuità informativa) e sulle azioni da implementare per garantire in maniera tempestiva il percorso di cura previsto (continuità gestionale). La continuità delle cure richiede anche buone relazioni di cura tra il paziente e il team che lo segue (continuità relazionale) $)^{26}$

La continuità dell'assistenza dovrebbe essere garantita soprattutto nelle aree socio-economicamente svantaggiate ${ }^{27}$

MI, Medicina Interna; VMD, valutazione multidimensionale. *Indicatori negativi di una gestione non coordinata sono dati dalla mancata aderenza del paziente al trattamento o da un'eccessiva variazione della terapia e del piano di cura originale proposto dall'équipe medica fin dall'inizio responsabile del caso. Questo fenomeno si può verificare quando: i) cambiano frequentemente i medici responsabili del paziente; ii) sono richieste troppe - inutili - consulenze; iii) il paziente si è rivolto spontaneamente o è stato inviato ad altri consulenti, servizi, ospedali per lo stesso problema; iv) i controlli previsti dal piano assistenziale sono stati insufficienti e il paziente è stato costretto, come se abbandonato, a rivolgersi ai servizi di emergenza. 
Le definizioni di comorbilità e multimorbilità sono riportate nella Tabella 3.

La prevalenza della multimorbilità nelle persone anziane varia dal 55 al 98\%. Questo dato epidemiologico impone la necessità di un approfondimento del problema, con la proposizione di ulteriori studi per definire le migliori strategie di gestione di questi pazienti. ${ }^{41}$

\section{La dipendenza funzionale}

In adulti più anziani la multimorbilità si associa più frequentemente alla disabilità. ${ }^{42}$ In uno studio effettuato su una popolazione svedese di oltre $1000 \mathrm{pa}-$ zienti di età superiore o uguale a 77 anni la presenza di disabilità funzionale è stata osservata nel 17,9\% dei partecipanti, con un incremento della sua prevalenza in rapporto all'aumento del numero di malattie croniche. La prevalenza di disabilità varia notevolmente secondo i diversi cluster di associazione di malattie: dal $6,7 \%$ nelle persone affette da ipertensione e fibrillazione atriale al $82,4 \%$ nelle persone affette da demenza e frattura dell'anca. ${ }^{43}$ La classificazione internazionale Impairments, Disabilities, and Handicaps (ICIDH) dell'Organizzazione Mondiale della Sanità ha definito la tassonomia delle conseguenze di una malattia con tre condizioni principali: invalidità, disabilità, handicap (Tabella 4). ${ }^{44}$ Un altro schema, sviluppato dal sociologo Saud Nugi, descrive quattro situazioni fondamentali: patologia attiva, invalidità, limitazione funzionale e disabilità. ${ }^{45,46}$

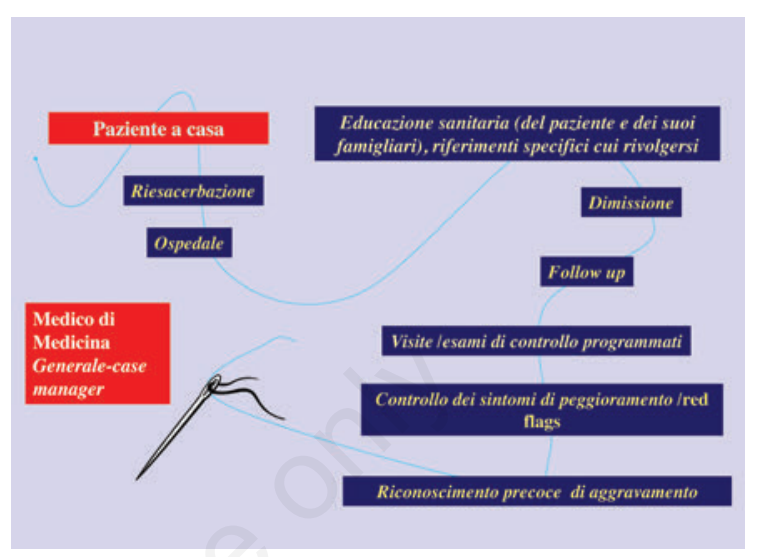

Figura 4. Coordinamento dell'assistenza nelle malattie croniche: bisogna facilitare le connessioni tra i servizi esistenti.

Tabella 3. Comorbilità e multimorbilità.

Comorbilità
Compresenza di un'ulteriore malattia in aggiunta ad specifica
malattia indice (disease-centered)
Le Comorbilità non sono direttamente legate alla diagnosi primaria,
ma possono coinvolgere qualsiasi altro organo o apparato

\section{Multimorbilità}

Coesistenza di diverse condizioni morbose nello stesso individuo Qualsiasi combinazione di malattie croniche con almeno un'altra malattia (acuta o cronica) o disfunzione bio-psico-sociale o fattore di rischio in grado di modificare l'impatto sulla prognosi, il consumo di risorse sanitarie e i risultati di salute, con possibile aumento della disabilità, della dipendenza funzionale e della fragilità, con evidente peggioramento della qualità di vita.

L'interesse si sposta pertanto da una data condizione patologica indice alla persona che soffre di patologie multiple (patient-centered)
Questa definizione comporta l'orientamento dell'interesse principal effetti di altri disturbi sulla prognosi della malattia indice

Fonti: Nobili et al., 2011; ${ }^{11}$ Feinstein, 1970; ${ }^{37}$ van den Akker et al., 1998; ${ }^{38}$ Batstra et al., 2002; ${ }^{39}$ Boyd et al., $2010 .{ }^{40}$

Tabella 4. Relazioni fra malattia attiva, menomazione, disabilità, handicap e limitazione funzionale.

Classificazione internazionale OMS Impairments, Disabilities, and Handicaps (ICIDH)

\begin{tabular}{lccc}
\hline Malattia & \multicolumn{1}{c}{ Menomazione } & Disabilità & Handicap \\
\hline $\begin{array}{l}\text { Patologia intrinseca o } \\
\text { condizione patologica } \\
\text { attuale }\end{array}$ & $\begin{array}{c}\text { Perdita o anormalità di una } \\
\text { struttura o di una funzione } \\
\text { fisiologica, anatomica oppure } \\
\text { psicologica (in questo caso } \\
\text { si tende a parlare di disturbo })\end{array}$ & $\begin{array}{c}\text { Limitazione o mancanza di capacità } \\
\text { di compiere un'attività }\end{array}$ & $\begin{array}{c}\text { Indica lo svantaggio sociale vissuto } \\
\text { da una persona a seguito di una } \\
\text { disabilità o menomazione, che limita } \\
\text { della menomazione }\end{array}$ \\
& $\begin{array}{c}\text { Indica lo svantaggio personale } \\
\text { che la persona disabile vive in } \\
\text { normale per la persona (dipende } \\
\text { funzione di ciò che è in grado di fare } \\
\text { o meno }\end{array}$ & $\begin{array}{c}\text { dall'età, dal sesso, da fattori socio culturali) } \\
\end{array}$ \\
& & \\
& &
\end{tabular}


La dipendenza funzionale si riferisce a persone non autosufficienti in almeno una delle attività della vita quotidiana (activity daily living, ADL: lavarsi, vestirsi, mangiare, andare o scendere dal letto, trasferirsi in sedia, mobilità , uso della toilette, continenza sfinterica) $\mathrm{o}$ in un'attività strumentale della vita quotidiana (instrumental activity daily living, IADL: prepararsi i pasti, fare la spesa, gestire il denaro, usare il telefono, fare lavori domestici, uscire di casa) (Tabelle 5-7).

\section{La fragilità}

Complessità e fragilità si possano sovrapporre. Frequentemente questi termini sono usati indifferentemente l'uno con l'altro, quando invece dovrebbero essere considerati separatamente. ${ }^{50,51}$ Non è facile definire la fragilità secondo un univoco concetto. Rockwood et al. hanno definito la fragilità come uno stato vulnerabile di salute, derivante dalla complessa interazione di problemi medici e sociali, con ridotta capacità di rispondere allo stress, associata ad una riduzione funzionale di performance. ${ }^{52}$ La persona anziana fragile, in generale, è un soggetto debole, di età avanzata o molto avanzata, con disabilità di differenti livelli di gravità e con presenza di sindromi geriatriche associate $^{\mathrm{b}}$ (Tabella 6).

Da un punto di vista più strettamente clinico, la fragilità degli anziani è caratterizzata da un'elevata suscettibilità a sviluppare malattie (spesso con un decorso clinico atipico, limitazione delle capacità motorie, propensione all'immobilità, rapide fluttuazioni dello stato di salute), eventi a cascata, rischio di eventi avversi e di complicanze, ridotta capacità di recupero, necessità di costante sorveglianza medica, aumentato rischio di mortalità Figura $5 .{ }^{53} \mathrm{La}$ Clinical Fraility Scale considera

b Le più comuni sono il delirium, i disturbi cognitivi, le ulcere da pressione, l'incontinenza sfinterica urinaria e/o fecale, le vertigini e l' instabilità dell'equilibrio, le cadute, i disturbi del visus e dell'udito, i disturbi alimentari con perdita di peso, le patologia jatrogene e nosocomiali. la valutazione delle malattie diagnosticate, la motivazione del paziente, il controllo dei sintomi, lo stato funzionale ed il grado di dipendenza. ${ }^{54}$

\section{La definizione di complessità}

Che cosa rende i nostri pazienti complessi e come possiamo misurare la loro complessità? Queste domande devono ricevere ancora, a tutt'oggi, una risposta esaustiva..$^{55}$

Nei pazienti ospedalizzati, la dizione complessità del case mix viene utilizzata dai clinici e dagli amministratori per descrivere una serie di attributi multipli che includono, più che la comorbilità di per sé, la severità della malattia, il rischio di morte, la prognosi, la difficoltà di trattamento, i bisogni assistenziali, l'entità delle risorse messe in campo, eccetera. Per i clinici essa viene riferita al paziente, ai suoi bisogni complessivi e alle modalità di garantirgli l'assistenza necessaria, secondo diversi aspetti: severità maggiore delle malattie, aumentato rischio di mortalità, difficoltà nella terapia e nella gestione dei pazienti, prognosi peggiore, necessità più

- Elevata suscettibilità a sviluppare malattie acute che si esprimono con quadri clinici atipici (confusione mentale, incontinenza urinaria, instabilità posturale e cadute, etc.)

- Ridotta capacità motoria, fino alla immobilità, per una grave astenia ed adinamia, non completamente giustificate dalle singole patologie presenti

- Fluttuazioni rapide dello stato di salute, con spiccata tendenza a sviluppare complicanze (scompenso a cascata)

- Elevato rischio iatrogeno e di eventi avversi

- Lenta capacità di recupero, comunque quasi sempre parziale

- Failure to thrive, spesso associate ad aumentata incidenza di infezioni, depressione dell'immunità cellulo-mediata, fratture di femore, ulcere da pressione, aumentata mortalità post-chirurgica

- Frequente richiesta di intervento medico, ripetute ospedalizzazioni, necessità di continuità assistenziale

- Alto rischio di morte

Figura 5. Conseguenze cliniche della fragilità. Fonte: Nardi, 2010. ${ }^{53}$

Tabella 5. La disabilità: diversi approcci possibili.

Attività della vita quotidiana

Approccio ambientale alla disabilità

funzionale adottato dalla WHO
Capacità elementari che permettono di muoversi con un minimo di autonomia e indipendenza, includendo qualsiasi attività quotidiana svolta per la cura di noi stessi, il lavoro, i lavori di casa, ed il tempo libero

Ci sono due principali gruppi di ADL: le attività di base, relative alla cura di sé, come lavarsi, vestirsi, mangiare, il controllo volontario degli sfinteri, camminare, e le attività strumentali, quali i lavori di casa leggeri, la preparazione di pasti, l'assunzione di farmaci, la spesa per generi alimentari o vestiti, l'uso del telefono e la gestione del denaro. Questo modello è stato utilizzato per sviluppare l'indice di Katz ${ }^{47}$ e l'indice di Barthel ${ }^{48}$ scala di valutazione standard per misurare la disabilità

Approccio bio-psico-sociale integrato, che considera tre componenti principali: le funzioni e le strutture corporee, le attività e la partecipazione sociale ed i fattori di contesto ambientale e personale. L'indipendenza è definita come la capacità di eseguire un'attività senza o con un minimo aiuto da parte di altri ${ }^{49}$ 
elevate di assistenza. Gli amministratori e i gestori tendono ad enfatizzare, nel concetto di complessità del case mix, il maggior consumo di risorse impiegate, con aumento dei costi delle cure. Il concetto di complessità non è univoco, estendendosi dalla sfera clinica a quella psico-sociale, da quella economica a quella organizzativa (Figura 6). ${ }^{56} \mathrm{Il}$ termine complessità (da complexus) rappresenta il legame tra l'unità e la molteplicità, contemplando insieme inseparabilità e differenza, incertezza, dinamicità, imprevedibilità, rischio. Di fronte al malato complesso non servono logiche centranti. Queste rischiano di essere semplificanti e riduttive. Sono necessarie logiche eccentriche, in grado di decentrare la conoscenza sulla multidimensionalità rispetto ai molti centri del malato. ${ }^{57}$

Il concetto di complessità manca di una sua precisa definizione. La complessità sottende una qualità intrinseca di essere variegata e intricata. Essa definisce il grado delle interrelazioni di ciascun componente di un sistema complesso, in funzione della loro numerosità, delle interfacce, delle condizioni contingenti, delle opzioni decisionali possibili. ${ }^{58} \mathrm{Un}$ sistema è complesso quando risulta composto da diverse, molteplici componenti interconnesse tra loro in maniere diverse. Un sistema complesso è caratterizzato da una grande quantità di attori/elementi/caratteristiche, da un cospicuo numero di loro possibili interazioni, è gravato da rischi (alea) rilevanti. ${ }^{59}$ La complessità è il mondo reale. Essa richiede una visione alternativa nell'assistenza sanitaria, basata su un equo giudizio delle dinamiche delle possibili interazioni tra $\mathrm{i}$ diversi componenti in causa . Complessità significa impossibilità di ridurre in termini lineari non tanto la realtà oggetto di studio da parte della scienza, ma i modi $d i$ versi in cui la realtà viene studiata. Il pensiero complesso aspira alla conoscenza multidimensionale e multidisciplinare, ma è consapevole dell'impossibilità della conoscenza completa. Il pensiero complesso è animato da una tensione permanente tra l'aspirazione a un sapere non parcellizzato, non settoriale, non riduttivo, orientato a sviluppare l'attitudine ad interconnettere le conoscenze, a contestualizzare la complessità nel mondo reale: esso richiede una visione competente nell'assistenza, basata su un equo giudizio delle possibili interazioni tra i diversi componenti in causa. ${ }^{60,61}$ La complessità rende la Medicina una scienza probabilistica, con un elevato rischio di errore, per l'incertezza che permea le decisioni mediche, che pur devono essere prese in un tempo limitato e in un contesto di conoscenza non sempre definito. ${ }^{61} \mathrm{La}$ definizione di paziente complesso, adottata dall'Agency for Healthcare Research and Quality's (AHRQ), si riferisce ad una persona con due o più malattie croniche, in cui ciascuna delle condizioni morbose presenti è in grado d'influenzare l'esito delle cure delle altre coesistenti, attraverso varie modalità: la limitazione della speranza di vita, l'aumentata morbilità intercorrente, le interazioni tra terapie farmacologiche, l'impossibilità al pieno impiego di cure adeguate per controindicazione, eccetera. ${ }^{62}$ Nell'accezione più completa di complessità le componenti di tipo biologico, socioeconomico, culturale, comportamentale ed ambientale diventano tutti importanti determinanti di salute. ${ }^{63}$ Purtroppo - e paradossalmente - questi elementi vengono considerati, nei criteri di eleggibilità/esclusione degli studi clinici come potenziali confondenti ai fini di una oggettiva valutazione dei risultati.

In Medicina Interna la gestione di un paziente complesso significa, in funzione delle sue caratteri-

\begin{tabular}{|l|}
\hline Severità dei sintomi, stabilità/instabilità della malattia \\
Difficoltà diagnostica \\
Presenza di dipendenza funzionale e/o disfunzione cognitiva \\
Capacità/incapacità/inerzia nell'assumere decisioni (decision making) \\
\hline Preferenze personali del paziente e dei familiari \\
Stress e preoccupazione per i sintomi, vissuto di malattia \\
Disponibilità al trattamento, capacità di adesione \\
\hline Sicurezza economica e stabilità domestica/residenziale \\
Contesto socio-famigliare \\
Presenza di caregivers \\
\hline Organizzazione delle cure (quanta, con quanti e quali attori, con quale \\
intensità) \\
Bisogno assistenziale \\
Coordinamento e continuità delle cure \\
Relazione medico (team di cura) e paziente \\
\hline
\end{tabular}

Figura 6. Le dimensioni della complessità in Medicina Interna. Fonte: Grant et al., 2011. ${ }^{56}$

Tabella 6. Caratteristiche della fragilità.

La fragilità descrive un fenotipo di persone anziane, con comorbilità ed instabilità clinica, aumentata disabilità e rischio di eventi avversi, con un'alta incidenza di ricoveri ripetuti e di morte. Tale condizione può associarsi a peggioramento della qualità della vita, compresa la disabilità e problemi socio-economici

I pazienti fragili sono meno resistenti: la loro capacità di recupero attraverso i normali meccanismi di compenso e di adattamento è compromessa

Essenzialmente, la fragilità deriva dalla riduzione delle riserve funzionali e di resistenza agli stress, in riferimento ad un declino cumulativo delle normali funzioni fisiologiche, con un aggiuntivo invecchiamento patologico al normale processo involutivo senile

Nell'anziano o nel paziente molto anziano, la fragilità è la condizione in cui la complessità dei pazienti rende la prognosi infausta e particolarmente onerosa dal punto di vista dei carichi assistenziali correlati alle cure 
stiche specifiche, possedere competenze peculiari per affrontare le sfide poste dal singolo caso ed il contesto (Figura 7; Tabella 7). Studiare la complessità significa comprendere il bisogno di ricomposizione, di riaggegazione delle conoscenze, superando la frammentarietà. ${ }^{64}$

I pazienti complessi rappresentano spesso una zona grigia e richiedono trattamenti su misura, in base ad un giudizio clinico idoneo, decisioni adeguate, secondo i massimi livelli di consapevolezza in relazione al contesto (situation awareness), ai fini della decisione più adeguata. Complessità delle cure può essere intesa anche come una maggiore quantità di tempo da dedicare al paziente per valutare e trattare l'assistenza necessaria, ${ }^{65}$ con un case mix variegato e un'ampia gamma di possibili decisioni da assumere (Tabelle 8 e 9; Figura 8). ${ }^{66}$

\section{Conclusioni}

La complessità costituisce la sfida quotidiana del medico internista. Pur se non prevista in maniera sistematica nella formazione del medico, essa è ben presente nel mondo reale, tanto che affrontare questo problema appare oggi doveroso ${ }^{68}$ Per ottenere risultati efficaci in questo ambito il medico internista deve saper ribadire le proprie capacità, ${ }^{69}$ con la valutazione sistemica dei bisogni e della prognosi dei pazienti, andando oltre le singole prestazioni, considerando il processo assistenziale nel suo insieme, filtrando le utili opinioni specialistiche con la regia dei casi, mediante un controllo ed un feed-back sistematico delle interazioni esistenti fra gli attori/elementi in causa e dei rischi possibili, anche in condizioni di incertezza. ${ }^{70,71}$ L'illusione della semplicità obbliga ad una revisione delle modalità con cui i medici affrontano oggi i pazienti complessi. ${ }^{3}$ Semplifi- care la complessità non significa banalizzare $\mathrm{o}$ affrontare i problemi in modo semplicistico. Un sociologo di oggi sostiene con grande acume che la semplicità è una complessità risolta (Domenico De Masi, Sociologo, intervista televisiva, 22 febbraio 2014). La valutazione globale dei problemi incentrata sul paziente, l'integrazione, la cooperazione e il coordinamento, la comunicazione efficace sono alcune semplici regole utili ad ottenere risultati tangibili in un sistema complesso. ${ }^{72,73}$ Questi, insieme alla ricerca mirata ad approfondire in maniera multidimensionale il tema della complessità, devono diventare gli strumenti del medico internista per poter riaffermare la sua naturale vocazione di medico della persona.

Tabella 7. Competenze e capacità dell'internista necessarie per la gestione del paziente complesso.

\begin{tabular}{ll}
\hline Caratteristiche dei pazienti internistici & Competenze e sfide per l'internista \\
\hline $\begin{array}{ll}\text { Generalmente anziani o molto anziani } \\
\text { con diverse comorbilità }\end{array}$ & Valutazione clinica, laboratoristica, strumentale e multidimensionale \\
esclusi dai grandi trials clinici & Inquadramento e valutazione del setting assistenziale \\
con prevalenti malattie croniche/cronico-riacutizzate & Consapevolezza della situazione, in funzione della prognosi \\
fragili a volte disabili, a rischio di perdita dell'autosufficienza & Stratificazione del rischio \\
a volte instabili, in alcuni casi critici con limitata speranza di vita & Selezione degli elementi salienti \\
in trattamento polifarmacologico & Definizione delle priorità \\
con frequenti ricoveri ripetuti & Gerarchizzazione dei bisogni \\
in alcuni casi a rischio di dimissione difficile & Selezione degli obiettivi di cura \\
che richiedono il giusto tempo di ascolto e di valutazione & Decision making in condizioni d'incertezza \\
condivisi fra diversi medici nel processo di assistenza & Stratificazione prognostica \\
che richiedono continuità assistenziale & Differenziazione nell'intensità delle cure \\
& Relazione di cura ed empatia con il paziente \\
& Relazioni con i caregivers \\
& Educazione al self management \\
& Coordinamento e comunicazione \\
& Pianificazione e care case management \\
& Continuità assistenziale
\end{tabular}


Tabella 8. Livelli di consapevolezza in funzione delle decisioni del medico internista.

\begin{tabular}{|c|c|c|}
\hline Livello I & Livello II & Livello III \\
\hline $\begin{array}{l}\text { Comporta la percezione delle informazioni } \\
\text { e degli spunti salienti dall'ambiente } \\
\text { Corrisponde al processo di acquisizione } \\
\text { delle informazioni attraverso l'anamnesi } \\
\text { e la storia clinica, l'esame fisico e i test } \\
\text { diagnostici }\end{array}$ & $\begin{array}{c}\text { È la capacità d'integrazione delle } \\
\text { informazioni che consente di prendere } \\
\text { le decisioni }\end{array}$ & $\begin{array}{c}\text { E' il più alto livello di comprensione } \\
\text { della situazione: } \\
\text { corrisponde alla capacità di prevedere } \\
\text { gli eventi futuri da parte di un esperto } \\
\text { qualificato in grado di valutare } \\
\text { gli elementi clinici, la letteratura scientifica, } \\
\text { il contesto }\end{array}$ \\
\hline \multicolumn{3}{|l|}{ Ai fini della decisione del medico: } \\
\hline $\begin{array}{l}\text { Quali sono gli elementi salienti } \\
\text { di questo paziente? }\end{array}$ & $\begin{array}{c}\text { Cosa sta succedendo a questo paziente? } \\
\text { Quali priorità? } \\
\text { Cosa dobbiamo fare? }\end{array}$ & Che cosa è più probabile che accada se ...? \\
\hline
\end{tabular}

Modificata da Singh et al., $2006{ }^{67}$

Tabella 9. Paziente internistico complesso, critico, instabile e discrezionalità decisionale.

\begin{tabular}{|c|c|c|c|}
\hline Paziente stabile complesso & Paziente instabile & & critico \\
\hline $\begin{array}{l}\text { Paziente che non presenta alterazioni dello stato } \\
\text { di coscienza, con parametri vitali e ABC } \\
\text { non alterati, con valori pressori normali, } \\
\text { con patologie pluri-organo, complesse, o } \\
\text { sistemiche o con più malattie coesistenti } \\
\text { nella stesso individuo }\end{array}$ & $\begin{array}{l}\text { Paziente che presenta alterazione } \\
\text { della coscienza, con compromissione } \\
\text { delle funzioni vitali }-\mathrm{ABC} \text {, con } \\
\text { ipotensione (PAS }<90 \mathrm{mmHg} \\
\text { nonostante riempimento) }\end{array}$ & $\begin{array}{r}\text { Paziente po } \\
\text { attuale o } \\
\text { acuta di un } \\
\text { o di più organi } \\
\text { rischio possib } \\
\text { osservazion }\end{array}$ & $\begin{array}{l}\text { i una condizione } \\
\text { l'insufficienza } \\
\text { /o di un sistema, } \\
\text { hi, con conseguente } \\
\text { vita, che richiede } \\
\text { lua e prestazioni } \\
\text { zzate }\end{array}$ \\
\hline Discrezionalità nelle decisioni & & & \\
\hline Paziente stabile complesso & Paziente instabile & & ente critico \\
\hline Alta & Bassa & & Alta \\
\hline $\begin{array}{l}\text { Possibile case mix } \\
\text { Rispetto alla complessità, stabilità e criticità d }\end{array}$ & aziente & & \\
\hline $\begin{array}{l}\text { Paziente critico } \\
\text { instabile }\end{array}$ & $\begin{array}{l}\text { Paziente non critico } \\
\text { ma a rischio di instabilità }\end{array}$ & $\begin{array}{l}\text { Paziente critico } \\
\text { stabilizzato }\end{array}$ & $\begin{array}{l}\text { Paziente critico } \\
\text { cronico }\end{array}$ \\
\hline
\end{tabular}

$\mathrm{ABC}$, compromissione delle vie aeree (A), del respiro (B), del circolo (C); PAS, pressione arteriosa sistolica.

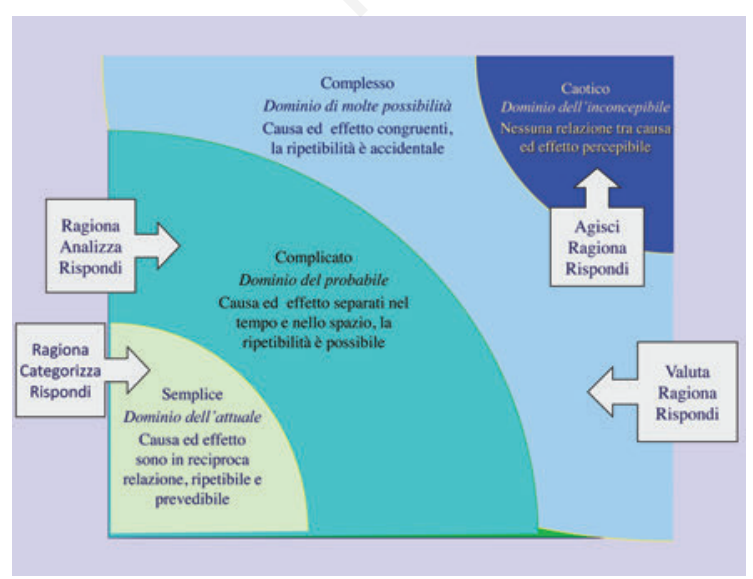

Figura 8. I domini della complessità in Medicina Interna. Modificata da Sturmberg et al., 2009. ${ }^{66}$

\section{Bibliografia}

1. Kane RL. The chronic care paradox. J Aging Soc Policy 2000;11:107-14.

2. Kane RL. Changing the face of long-term care. J Aging Soc Policy 2005;17:1-18.

3. Weiner H. The illusion of simplicity: the medical model revisited. Am J Psychiatry 1978;135:27-33.

4. Nardi R, Scanelli G, Borioni D, et al. The assessment of complexity in internal medicine patients. The FADOI Medicomplex Study. Eur J Intern Med 2007;18:283-7.

5. Nardi R, Scanelli G. Multimorbidity and complexity: a current research priority not only in the UK and in primary care, but all over the world and in every care setting. BMJ 2007;334:1016.

6. Nardi R, Scanelli G, Corrao S, et al. Co-morbidity does not reflect complexity in internal medicine patients. Eur J Intern Med 2007;18:359-68. 
7. Nardi R, Corbetta L, Muratori M, et al. Metodologia clinica, strumenti di valutazione e gestione dei pazienti anziani affetti da BPCO e comorbilità croniche. Ital J Med 2011;5:S171-8.

8. Leipzig RM, Hall WJ, Fried LP. Treating our societal scotoma: the case for investing in geriatrics, our nation's future, and our patients. Ann Intern Med 2012;156:657-8.

9. Bernardini B, Meinecke C, Zaccarini C, et al. Adverse clinical events in dependent long-term nursing home residents. J Am Geriatr Soc 1993;41:105-11.

10. Bernardini B, Meinecke C, Pagani M, et al. Epidemiology of adverse clinical events (ACES) as a dynamic measure of geriatric care management. Aging 1995;7: 191-3.

11. Nobili A, Licata G, Salerno F, et al. Polypharmacy, length of hospital stay, and in-hospital mortality among elderly patients in internal medicine wards. The REPOSI study. Eur J Clin Pharmacol 2011;67:507-19.

12. Weiner SJ, Schwartz A, Weaver F, et al. Contextual errors and failures in individualizing patient care. Ann Intern Med 2010;153:69-75.

13. Croskerry P, Shapiro M, Campbell S, et al. Profiles in patient safety: medication errors in the emergency department. Acad Emerg Med 2004;11:289-99.

14. Biel M. Reconceptualizing certified practice. Aliso Viejo, CA: AACN Certification Corporation; 1997.

15. Cei M, Bartolomei C, Mumoli N. In-hospital mortality and morbidity of elderly medical patients can be predicted at admission by the modified early warning score: a prospective study. Int J Clin Pract CME 2009; 63:591-5.

16. Chesi G, Nardi R. Severity stratification of patients hospitalized on the Internal Medicine ward: work in progress. Ital J Med 2013;7:231-3.

17. Katerndahl DA, Wood R, Jaén CR. A method for estimating relative complexity of ambulatory care. Ann Fam Med 2010;8:341-7.

18. Steiger WA. Managing difficult patients. Ann Intern Med 1965;62:1083.

19. Meschi T, Fiaccadori E, Cocconi S, et al. [Analysis of the problem of difficult hospital discharges in the University Hospital of Parma]. Ann Ital Med Int 2004;19:109-17. [Article in Italian]

20. Nardi R, Scanelli G, Tragnone A, et al. Difficult hospital discharges in internal medicine wards. Intern Emerg Med 2007:2:95-9.

21. Jencks SF, Williams MV, Coleman EA. Rehospitalizations among patients in the medicare fee-for-service program. N Engl J Med 2009;360:1418-28.

22. Wagner EH. Chronic disease management: what will it take to improve care for chronic illness? Effect Clin Pract 1998;1:2-4.

23. Wedding U. Endpoints and their relevance to older people, cancer and palliative care and work of EORTC. Elderly Task Force EORTC. Brussels: University of Jena; 2012.

24. Department of Health. Modern standards and service models - National service framework for older people, March 2001. London: Department of Health; 2001. Available from: http://www.dh.gov.uk/prod_consum dh/groups/dh_digitalassets/@dh/@en/documents/digitalasset/dh_4071283.pdf

25. Haggerty JL, Reid RJ, Freeman GK, et al. Continuity of care: a multidisciplinary review. BMJ 2003;327:1219-21.
26. Haggerty JL. Ordering the chaos for patients with multimorbidity. BMJ 2012;345:e5915.

27. Barnett K, Mercer SW, Norbury M, et al. Epidemiology of multimorbidity and implications for healthcare, research, and medical education: a cross-sectional study. Lancet 2012;380:37-43.

28. Stange KC. In this issue: assessing and acting on complexity. Ann Fam Med 2012;10:98-9.

29. Akker M, van den Buntinx F, Knottnerus JA. Comorbidity or multimorbidity: what's in a name? A review of literature. Eur J Gen Pract 1996;2:65-70

30. Fried LP, Ferrucci L, Darer J, et al. Untangling the concepts of disability, frailty, and comorbidity: implications for improved targeting and care. J Gerontol A Biol Sci Med Sci 2004;59:255-63.

31. Guralnik JM. Assessing the impact of comorbidity in the older population. Ann Epidemiol 1996;6:376-80.

32. Canadian Institute for Health Information Partnership for Health Informations Standards. WHIC Chronic Disease Management Infostructure. CDM data standards; Fall 2005. Symposium. Available from: http://secure.cihi.ca/ cihiweb/en/downloads/WHIC_CDM_Management Infostructure_CDM_Data_Standards_Peter_Sargious.pdf

33. Huntley AL, Johnson R, Purdy S, et al. Measures of multimorbidity and morbidity burden for use in primary care and community settings: a systematic review and guide. Ann Fam Med 2012;10:134-41.

34. Fortin M, Stewart M, Poitras M, et al. A systematic review of prevalence studies on multimorbidity: toward a more uniform methodology. Ann Fam Med 2012; 10:142-51.

35. Bayliss EA, Ellis JL, Shoup JA, et al. Association of patient-centered outcomes with patient-reported and ICD9-based morbidity measures. Ann Fam Med 2012; 10:126-33.

36. Le Reste JY, Nabbe P, Manceau B, et al. The European General Practice Research Network presents a comprehensive definition of multimorbidity in family medicine and long term care, following a systematic review of relevant literature. J Am Med Dir Assoc 2013;14:319-25.

37. Feinstein AR. The pre-therapeutic classification of comorbidity in chronic disease. J Chronic Dis 1970;23: 455-68.

38. van den Akker M, Buntinx F, Metsemakers JF, et al. Multimorbidity in general practice: prevalence, incidence, and determinants of co-occurring chronic and recurrent diseases. J Clin Epidemiol 1998;51:367-75.

39. Batstra L, Bos EH, Neeleman J. Quantifying psychiatric comorbidity - Lessons from chronic disease epidemiology. Soc Psychiatry Psychiatr Epidemiol 2002;37;105-11.

40. Boyd CM, Fortin M. Future of multimorbidity research: how should understanding of multimorbidity inform health system design? Public Health Rev 2010;32:451-74.

41. Marengoni A, Angelman S, Melis R, et al. Aging with multimorbidity: a systematic review of the literature. Ageing Res Rev 2011;10:430-9.

42. Newman AB. Comorbidity and multimorbidity. In: Newman AB, Cauley JA, eds. The epidemiology of aging. Dordrecht: Springer-Verlag; 2012. pp 119-133.

43. Marengoni A, Angleman S, Fratiglioni L. Prevalence of disability according to multimorbidity and disease clustering: a population-based study. J Comorbidity 2011;1:11-8. 
44. World Health Organization. The International Classification of Impairments, Disabilities, and Handicaps (ICIDH) defined a taxonomy of disease impacts by the World Health Organization. Geneva: World Health Organization; 1980.

45. Nagi SZ. Disability concepts revised: implications for prevention. In: Pope AM, Tarlov AR, eds. Disability in America: toward a national agenda for prevention. Washington, DC: National Academy Press; 1991. Pp 309-327.

46. Salvador-Carulla L, Gasca VI. Defining disability, functioning, autonomy and dependency in person-centered medicine and integrated care. Int $\mathrm{J}$ Integr Care 2010;10:e25.

47. Katz S. Assessing self-maintenance: activities of daily living, mobility, and instrumental activities of daily living. J Am Geriatr Soc 1983;31:721-7.

48. Mahoney FI, Barthel DW. Functional evaluation: the Barthel index. Md State Med J 1965;14:61-5.

49. World Health Organization. A glossary of terms for community healthcare and services for older persons. Ageing and Health Technical Report, Vol. 5, WHO/WKC/Tech.Ser./04.2. Kobe (Japan): WHO Centre for Health Development; 2004. Available from: http://www.who.int/kobe_centre/ageing/ahp_vol5_glossary.pdf Accessed: 14 April 2009.

50. Cerimele JM, Peccoralo LA. Defining patient complexity. Ann Intern Med 2012;156:606-7; author reply 607.

51. Olde Rikker MG, Schers HJ, Melis RJ. Defining patient complexity. Ann Intern Med 2012;156:606; author reply 607.

52. Rockwood K, Stadnyk K, Carver D, et al. A clinimetric evaluation of specialized geriatric care for rural dwelling, frail older people. J Am Geriatr Soc 2000;48:1080-5.

53. Nardi R. L'anziano fragile. Cap. 21. In: Mongardi M, ed. L'assistenza all'anziano - Ospedale, territorio, domicilio. Milano: Mcgraw-Hill; 2010. pp 351-367.

54. Rockwood K, Song X, MacKnight C, et al. A global clinical measure of fitness and frailty in elderly people. CMAJ 2005;173:489-95.

55. Turner BJ, Cuttler L. The complexity of measuring clinical complexity. Ann Intern Med 2011;155:851-2.

56. Grant RW, Ashburner JM, Hong CS, et al. Defining patient complexity from the primary care physician's perspective: a cohort study. Ann Intern Med 2011;155: 797-804.

57. Cavicchi I. Medicina Interna, paziente complesso: verso una clinica relazionale e ragionevole. Ital J Med 2012;6:259-64.

58. Evans T, Michael W, Marciniak J. Software quality assurance and management. New York, NY: John Wiley \& Sons Inc; 1987.

59. Morin E. Introduzione al pensiero complesso. Milano: Sperling \& Kupfer; 1993.

60. Wilson T, Holt T, Greenlagh T. Complexity and clinical care. Br Med J 2001;323:685-8.

61. Plsek PE, Wilson T. Complexity, leadership, and management in healthcare organisations. BMJ 2001;323:746-9.

62. Agency for Healthcare Research and Quality (AHRQ). Definition: Complex Patient, Funding Opportunity Announcement (FOA) - Technical Assistance Conference Call, October 15, 2007.

63. Safford MM, Allison JJ, Kiefe CI. Complexity: more than comorbidity. The vector model of complexity. J Gen Intern Med 2007;22:382-90.

64. Whittle J, Bosworth H. Studying complexity is complex. J Gen Intern Med 2007;22:379-81.

65. Upshur REG. Chronicity and complexity, Is what's good for the diseases always good for the patients? Can Fam Physician 2008;54:1655-8.

66. Sturmberg JP, Martin CM. Complexity and health-yesterday's traditions, tomorrow's future. J Eval Clin Pract 2009; 15:543-8.

67. Singh H, Petersen LH, Thomas EJ. Understanding diagnostic errors in medicine: a lesson from aviation. Qual Health Saf Care 2006;15:159-64.

68. Gensini GF, Fabbri LM, Fini M, Nozzoli C (a cura di). La Medicina della complessità: BPCO e comorbidità. Firenze: Firenze University Press; 2010.

69. Mazzone A, Nozzoli C, Berti F, et al. The clinical competence in internal medicine. Ital J Med 2011;5, Suppl 1, XVI Congressso nazionale FADOI, Firenze, 15-18 maggio 2011.

70. Fraser SW, Greenhalgh T. Coping with complexity: educating for capability. BMJ 2001;323:799-803.

71. Carmel M, Martin CM, Sturmberg JP. General practice chaos, complexity and innovation. MJA 2005;183:106-9.

72. Hammerly M. Commentary: patient-centered care, the sine qua non of collaborative medicine. Am J Med Qual 2002;17:33-8.

73. Ross GS. The future of generalism in medicine. Ann Intern Med 2005;143:693. 


\title{
Strumenti di valutazione della complessità nel paziente internistico: un percorso ancora da tracciare
}

\author{
Stefania Frasson, ${ }^{1}$ Roberto Nardi ${ }^{2}$ \\ ${ }^{1}$ Dipartimento per la Ricerca Clinica, Fondazione FADOI, Milano; ${ }^{2}$ Medicina Interna, Bologna, Italia
}

\section{RIASSUNTO}

La complessità del paziente anziano ricoverato in Medicina Interna rende necessario un approccio multidimensionale, in grado di indirizzare il medico ad una gestione ottimale del paziente. Per tale motivo Fondazione FADOI intende promuovere un progetto di studio con l'obiettivo di sviluppare e validare possibili strumenti in grado di descrivere la complessità del paziente ricoverato in Medicina Interna e le possibili correlazioni con gli esiti ottenuti nell'assistenza. La valutazione della complessità del paziente internistico dovrebbe essere in grado di misurare una serie di domini quali: comorbidità, stabilità clinica, fragilità sociale, disfunzione cognitiva, depressione, dipendenza funzionale, nutrizione, ulcere da pressione, dolore, aderenza alla terapia, politerapie, eccetera. Un approfondimento di questo tipo potrà generare informazioni utili ad ottimizzare le potenzialità e l'appropriatezza assistenziale della fase acuta, della post-acuzie e della cronicità delle malattie, nonchè a gestire la continuità di cure nella delicata transizione ospedale-territorio.

\section{Razionale}

Si stima che tra il 2006 e il 2050 il numero di anziani nel mondo sia destinato a duplicarsi, ${ }^{1}$ con conseguente aumento di tutte quelle condizioni fisio-patologiche che si accompagnano all'età avanzata. La Medicina Interna rappresenta oggi il contesto ospedaliero in cui maggiormente confluisce la gran parte di questi pazienti anziani (età $\geq 65$ anni) e molto anziani (età $\geq 80$ anni), che soffrono di condizioni di comorbidità, sia di tipo acuto ma più frequentemente cronico, spesso con limitazioni del loro grado di autonomia. ${ }^{2}$ In questi pazienti una valutazione puntuale del grado di comorbidità è necessaria, in quanto può permettere al medico di intervenire in maniera efficace sulla condizione del paziente ricoverato. La letteratura oggi ha in parte superato la rappresentatività omnicomprensiva della comorbidità nel paziente anziano, proponendo un concetto più allargato che coinvolga l'interazione di una serie di sistemi, tra i quali la comorbidità stessa, e che sia realmente indicativo del

Corrispondente: Stefania Frasson, Centro Studi FADOI, p.zza Cadorna 15, 20123 Milano, Italia.

E-mail: stefania.frasson@fadoi.org

Parole chiave: complessità, Medicina Interna, questionari, scores, comorbidità.

Articolo pubblicato secondo la Creative Commons Attribution NonCommercial 3.0 License (CC BY-NC 3.0).

C Copyright S. Frasson e R. Nardi, 2014

Licensee PAGEPress, Italy

QUADERNI - Italian Journal of Medicine 2014; 2:14-24 burden posto dal paziente anziano ricoverato in Medicina Interna. ${ }^{3}$ Questo nuovo concetto, conosciuto con il termine di complessità, non solo non è di semplice definizione, ma ancor meno è facilmente misurabile nella pratica clinica giornaliera; oltretutto, questi pazienti anziani e polipatologici sono spesso sotto-rappresentati e sotto-studiati nei trials di valutazione dell'efficacia di modelli gestionali nelle malattie croniche, proprio a causa della loro complessità. Nei pazienti complessi si rende necessario un approccio di sistema, in quanto la sola analisi delle singole componenti tende a costruire una serie di verità che non necessariamente rimangono tali quando i vari elementi vengono considerati nel loro insieme. ${ }^{4}$ Prendersi cura di un paziente complesso significa pertanto considerare la totalità dei problemi clinici e non, che, direttamente o indirettamente, condizionano il destino prognostico del paziente (Figura 1). ${ }^{5}$

Nonostante il crescente interesse verso nuovi modelli di gestione delle malattie croniche e la disponibilità di diversi strumenti per un approccio multidimensionale alla valutazione del paziente, la modalità di misurazione della complessità è ancora una questione irrisolta. ${ }^{6}$ La letteratura mette a disposizione alcuni strumenti per la valutazione della complessità nei pazienti medici, tra cui la scheda VAOR, l'indice PROFUND. ${ }^{7}$ e l'MPI score. ${ }^{8}$ Questi non risultano però di semplice utilizzo nella pratica clinica quotidiana, soprattutto in termini di tempo necessario per la loro compilazione. Sarebbe quindi auspicabile lo sviluppo di un metodo di valutazione multi-dimensionale più user-friendly e con elevato grado predittivo dell'outcome del paziente. In tale prospettiva, Fondazione FADOI intende promuovere un progetto di studio con l'obiettivo di: 
- descrivere la complessità nei pazienti ricoverati in Medicina Interna attraverso la definizione di un nuovo modello predittivo (COMPLIMED score), auspicabilmente di più agevole applicazione rispetto ai modelli esistenti e costruito attraverso una sofisticata tecnica di analisi statistica (analisi delle componenti principali);

- correlare il COMPLIMED score con misure di outcome (in relazione al ricovero e ad un periodo di follow-up di 1 anno dopo il ricovero ospedaliero indice);

- confrontare il COMPLIMED score con uno strumento già validato (MPI score).

Lo sviluppo di un nuovo metodo almeno equi-efficace in termini predittivi, ma più semplice rispetto agli esistenti potrebbe facilitare la caratterizzazione della complessità dei pazienti nella pratica clinica quotidiana, favorendo i processi decisionali ed una ottimizzazione dell'organizzazione sanitaria (sia intrache extraospedaliera). Le informazioni raccolte per ciascun paziente, che permetteranno poi l'effettivo sviluppo e validazione del tool multidimensionale, sono le seguenti: i) caratteristiche generali dei pazienti (età, sesso, indice di massa corporea); ii) terapia farmacologica, in termini quantitativi e qualitativi; iii) esami del sangue di routine; iv) eventuali episodi di delirio; v) eventuale numero di cadute; vi) vari questionari/strumenti relativi ai domini identificati come rappresentativi della complessità di un paziente:

- Comorbidità: comorbidity index rating scale (CIRS), Charlson score;

- Stabilità clinica: modified early warning score (MEWS);

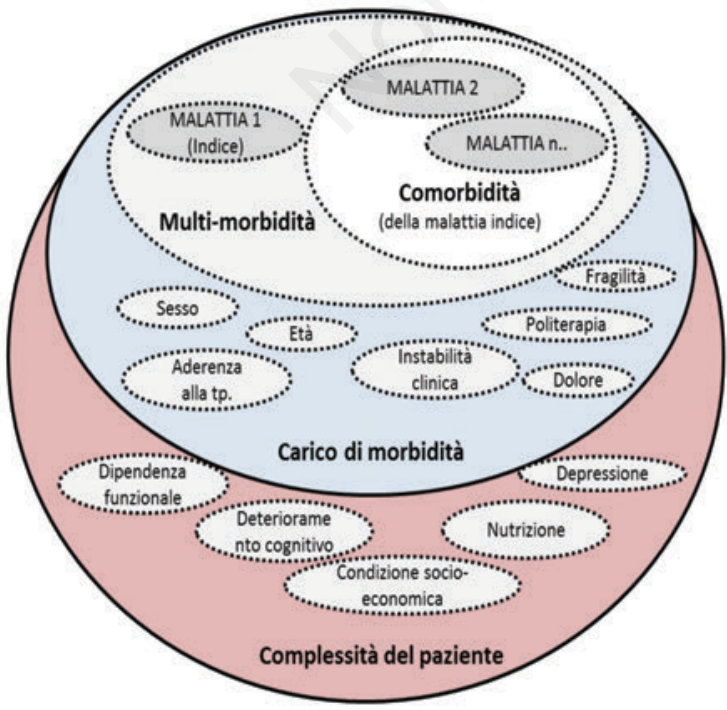

Figura 1. Il sistema complessità. Modificato da Gensini et al., $2010 .^{5}$
- Fragilità sociale: Flugelman score;

- Disfunzione cognitiva: short portable mental status questionnaire (SPMSQ);

- Depressione: 5-item geriatric depression scale (GDS);

- Dipendenza funzionale: Barthel, activity daily living (ADL), instrumental activity daily living (IADL);

- Nutrizione: mini nutritional assessment (MNA);

- Ulcere da pressione: scala Exton-Smith;

- Valutazione del dolore: numerical pain scale (NRS);

- Aderenza alla terapia: scala di Morisky.

Obiettivo del presente lavoro è una revisione puntuale dei principali strumenti di valutazione dei domini sopra citati e rappresentativi del sistema complessità, focalizzando l'attenzione su quelli sviluppati con sufficiente rigore metodologico e maggiormente utilizzati.

\section{La comorbidità e la multimorbidità}

Si definisce comorbidità la presenza di ogni patologia distinta, preesistente o coesistente, rispetto alla malattia indice, ovvero la malattia che determina un peggioramento dello stato di salute in un individuo, e/o l'evento acuto o la malattia che ne condiziona maggiormente la prognosi. Si differenzia dal concetto di multimorbidità che rappresenta più semplicemente la concomitanza di due o più malattie mediche nello stesso individuo. ${ }^{9}$ La prevalenza della multimorbidità, pur variando a seconda della casistica considerata e degli studi condotti, può essere stimata intorno al 20$30 \%$ della popolazione generale; questo dato sale dal $55 \%$ al $98 \%$ se si considera la popolazione anziana o le classi sociali disagiate. ${ }^{4}$ Multimorbidità e comorbidità rivestono un ruolo chiave nella valutazione globale del paziente complesso, non solo per la dimostrata correlazione ad indicatori prognostici quali durata della degenza, outcome e sopravvivenza, ma anche per il forte impatto sul sistema sanitario in termini di utilizzo delle risorse e costi associati. ${ }^{10} \mathrm{Si}$ rende pertanto necessaria una valutazione affidabile del livello di comorbidità del paziente ricoverato in Medicina Interna, con strumenti di misurazione validati.

\section{Gli strumenti di valutazione della comorbidità}

I maggiori strumenti di valutazione delle comorbidità sono riassunti in Tabella 1. ${ }^{11-14}$

\section{Cumulative illness rating scale}

Il CIRS ${ }^{11}$ è uno strumento standardizzato per ottenere una misura della salute somatica dell'anziano. Questo strumento richiede che il medico definisca la severità clinica e funzionale di 14 categorie di patologie. Il medico, sulla base della storia clinica, dell'e- 
same obiettivo e della sintomatologia presentata, definisce il livello di gravità per ognuna delle categorie (Tabella 2).

Si ottengono due indici:

1. Indice di comorbidità, che rappresenta il numero delle categorie nelle quali si ottiene un punteggio superiore o uguale a 3 (escludendo la categoria patologie psichiatriche/comportamentali);

2. Indice di severità, che risulta dalla media dei punteggi delle prime 13 categorie (escludendo la categoria patologie psichiatriche/comportamentali).

\section{Indice di Charlson}

Trattasi del più noto e più utilizzato indice di comorbidità (Tabella 3).$^{12}$ L'indice è costruito in base alla presenza nel singolo paziente di 19 patologie traccianti, ciascuna delle quali contribuisce allo score con un punteggio tra 1 e 6; la somma dei punteggi da malattie coesistenti viene poi ponderata per l'età: da 1 (50-59 anni) a 5 (90-99 anni). Nel lavoro originale di Charlson la validazione dello score su una casistica di pazienti neoplastici rivelava che uno score $>3$ era associato ad una probabilità di sopravvivenza del $45 \%$ in 10 anni. ${ }^{12}$ Tale indice è stato utilizzato soprattutto in pazienti anziani affetti da neoplasie, ${ }^{15}$ da malattia di Alzheimer ${ }^{16}$ ed in pazienti anziani sottoposti a interventi cardiochirurgici. ${ }^{17}$

\section{Fragilità: biologica, sociale e clinica}

L'ambiguità della definizione di fragilità riflette non solo la difficoltà a descrivere una sindrome clinica di cui non si conoscono compiutamente l'eziologia e

Tabella 1. Maggiori strumenti di valutazione delle comorbidità.

\begin{tabular}{ll}
\hline Indice & Bibliografia \\
\hline CIRS - cumulative illness rating scale & Parmalee et al., 1995 \\
\hline Indice di Charlson & Charlson et al., 1994 \\
\hline ICED - index of co-existent disease & Greenfield et al., 1995 \\
\hline Indice di Kaplan-Feinstein & Kaplan and Fenstein, $1974^{14}$ \\
\hline
\end{tabular}

Tabella 2. Cumulative illness rating scale score.

\begin{tabular}{|c|c|c|c|c|c|}
\hline 1. Patologie cardiache (solo cuore) & 1 & 2 & 3 & 4 & 5 \\
\hline 2. Ipertensione (si valuta la severità, gli organi coinvolti sono considerati separatamente) & 1 & 2 & 3 & 4 & 5 \\
\hline 3. Patologie vascolari (sangue, vasi, midollo, milza, sistema linfatico) & 1 & 2 & 3 & 4 & 5 \\
\hline 4. Patologie respiratorie (polmoni, bronchi, trachea sotto la laringe) & 1 & 2 & 3 & 4 & 5 \\
\hline 5. Occhi/O.R.L. (occhio, orecchio, naso, gola, laringe) & 1 & 2 & 3 & 4 & 5 \\
\hline 6. Apparato gastroenterico superiore (esofago, stomaco, duodeno, vie biliari epato-pancreatiche; escluso diabete) & 1 & 2 & 3 & 4 & 5 \\
\hline 7. Apparato gastroenterico inferiore (intestino, ernie) & 1 & 2 & 3 & 4 & 5 \\
\hline 8. Patologie epatiche & 1 & 2 & 3 & 4 & 5 \\
\hline 9. Patologie renali (solo rene) & 1 & 2 & 3 & 4 & 5 \\
\hline 10. Altre patologie genito-urinarie (ureteri, vescica, uretra, prostata e genitali) & 1 & 2 & 3 & 4 & 5 \\
\hline 11. Sistema muscolo-scheletrico-cute & 1 & 2 & 3 & 4 & 5 \\
\hline 12. Patologie sistema nervoso (s.n.c. e periferico, esclusa demenza) & 1 & 2 & 3 & 4 & 5 \\
\hline 13. Patologie endocrino-metaboliche (include diabete, sepsi, stati tossici) & 1 & 2 & 3 & 4 & 5 \\
\hline 14. Patologie psichiatriche e comportamentali (include demenza, depressione, ansia, agitazione, psicosi) & 1 & 2 & 3 & 4 & 5 \\
\hline \multicolumn{6}{|c|}{$\begin{array}{l}\text { 1=Assente - Nessuna compromissione d'organo/sistema; } \\
\text { 2=Lieve - La compromissione d'organo/sistema non interferisce con la normale attività; il trattamento può essere richiesto oppure no; la prognosi è eccellente } \\
\text { (esempi possono essere abrasioni cutanee, ernie, emorroidi); } \\
\text { 3=Moderata - La compromissione d'organo/sistema interferisce con la normale attività; il trattamento è necessario; la prognosi è buona (esempi possono essere co- } \\
\text { lelitiasi, diabete o fratture); } \\
\text { 4=Grave - La compromissione d'organo/sistema produce disabilità; il trattamento è indilazionabile; la prognosi può non essere buona (esempi possono essere car- } \\
\text { cinoma operabile, enfisema polmonare, scompenso cardiaco); } \\
5=\text { Molto grave - La compromissione d'organo/sistema mette a repentaglio la sopravvivenza; il trattamento è urgente; la prognosi è grave (esempi possono essere } \\
\text { infarto del miocardio; stroke; sanguinamenti gastro-intestinali; embolia). }\end{array}$} \\
\hline
\end{tabular}


la fisiopatologia, ma anche a riconoscerne i confini, che comprendono componenti mediche, funzionali, sociali, eccetera. ${ }^{18}$ Diversi sono i paradigmi che definiscono la fragilità:

- Il paradigma biomedico. Fried et al. ${ }^{19}$ hanno definito la fragilità come una sindrome fisiologica caratterizzata dalla riduzione delle riserve funzionali e dalla diminuita resistenza agli stressors risultante dal declino cumulativo di sistemi fisiologici multipli che causano vulnerabilità e conseguenze avverse.

- Il paradigma bio-psico-sociale. Gobbens et al. ${ }^{20} \mathrm{de}-$ finiscono la fragilità come uno stato dinamico che colpisce un individuo che sperimenta perdite in uno o più domini funzionali (fisico, psichico, sociale), causate dall'influenza di più variabili che aumentano il rischio di risultati avversi per la salute.

- Frailty index. Una definizione alternativa di fragilità è stata proposta da Rockwood et al. ${ }^{21}$ come frailty index (FI), costruito contando il numero di deficit accumulati nel tempo, all'interno di una lista molto ampia. Questa definizione è basata sull'idea che la fragilità sia uno stato di disorganizzazione caotica dei sistemi fisiologici che può essere stimata valutando lo stato funzionale, le malattie, i deficit fisici e cognitivi, i fattori di rischio psicosociali e le sindromi geriatriche, nell'ottica di costruire un quadro il più possibile completo del profilo di rischio di eventi avversi. ${ }^{22}$

Di fatto, la fragilità è una condizione di maggiore esposizione al rischio dell'anziano dovuta alla maggiore frequenza con cui l'anziano è soggetto ad incidenti o limitazioni. L'invecchiamento di per sé rappresenta una condizione di fragilità e induce alla cura e all'attenzione.

\section{Gli strumenti di valutazione della fragilità}

Non sono disponibili, a tutt'oggi, strumenti univoci e condivisi per valutare la fragilità; tuttavia si possono considerare come i maggiori strumenti di valutazione della fragilità gli indici riassunti in Tabella $4 .{ }^{23-29}$

\section{Indice di Flugelman}

L'indice clinico-sociale prognostico di Flugelman, proposto per la valutazione dei pazienti ospedalizzati, è basato sulla valutazione di sette parametri, comprendenti la mobilità, il controllo degli sfinteri, le capacità cognitive, l'alimentazione, la presenza di ulcere da pressione, le condizioni cliniche, il contesto familiare. La somma dei punteggi di tutti gli items definisce l'indice prognostico finale, che costituisce un mezzo semplice e abbastanza accurato per la valutazione della

Tabella 3. Indice di Charlson.

\begin{tabular}{|c|c|}
\hline Infarto miocardio & 1 \\
\hline Scompenso cardiaco & 1 \\
\hline Arteriopatia periferica (incluso aneurisma aorta $\geq 6 \mathrm{~cm}$ ) & 1 \\
\hline Malattie cerebrovascolari (accidente cerebrovascolare senza o con lievi deficit residui/TIA) & 1 \\
\hline Demenza & 1 \\
\hline Malattie polmonari croniche & 1 \\
\hline Malattie del tessuto connettivo & 1 \\
\hline Ulcera peptica & 1 \\
\hline Lievi malattie del fegato & 1 \\
\hline Diabete (senza danno d'organo) & 1 \\
\hline Diabete con danno d'organo & 2 \\
\hline Emiplegia & 2 \\
\hline Moderate o gravi malattie renali & 2 \\
\hline Tumore non metastatico (diagnosi $<5$ anni) & 2 \\
\hline Leucemia acuta o cronica & 2 \\
\hline Linfoma & 2 \\
\hline Moderate o gravi malattie del fegato & 3 \\
\hline Tumore solido metastatico & 6 \\
\hline AIDS (escluso HIV+) & 6 \\
\hline
\end{tabular}


prognosi dei pazienti. Un punteggio di 17 o più individua i pazienti a prognosi peggiore, sia in termini di prolungamento della degenza in ospedale che di maggiore mortalità. ${ }^{23}$ L'indice di Flugelman è stato proposto recentemente per identificare nei reparti internistici i pazienti più problematici, che richiedono una più prolungata degenza e che necessitano di una migliore pianificazione della dimissione. ${ }^{30}$

\section{(In)stabilità clinica}

Non esiste una definizione univoca di instabilità clinica. La letteratura fa riferimento ad una condizione clinicamente precaria, che comporta un intervento assistenziale di entità variabile in rapporto al grado di instabilità. L'instabilità clinica, per lo più valutata 24 ore prima della dimissione, è fattore prognostico di outcome sfavorevole a 30 giorni, soprattutto in termini di mortalità e ri-ospedalizzazioni. Ad oggi esistono vari strumenti in grado di definire e valutare tale condizione. I principali sono essenzialmente i seguenti:

i) Scala Halm: I criteri di Halm, messi a punto originariamente per i pazienti affetti da polmonite ricoverati in luoghi di cura acuta e rivisti in ambienti geriatrici riabilitativi, prevedono: temperatura corporea $>37,8$ gradi; frequenza cardiaca $>100 / \mathrm{min}$; frequenza respiratoria $>24 / \mathrm{min}$; saturazione di ossigeno: $<90 \%$; incapacità ad alimentarsi in modo autonomo; delirium; dolore.

Il riscontro di instabilità clinica deriva dalla presenza di almeno uno tra i primi cinque indicatori. Gli ultimi due indicatori, definiti di stato, devono essere associati ad almeno uno dei primi cinque per poter definire una persona clinicamente instabile. ${ }^{31}$

ii) Scala SIC, scala di instabilità clinica: La scala di instabilità clinica (SIC) consente di classificare la frequenza con cui viene effettuato il monitoraggio medico-infermieristico in setting di lungodegenza. I pazienti possono essere classificati in 5 categorie (da $0=$ stabile a $4=$ altamente instabile) in funzione della presenza o meno di necessità di monitoraggio medico-infermieristico e/o strumentale, e della frequenza con la quale tale monitoraggio deve essere condotto. ${ }^{32}$

iii) Scala MEWS, modified early warning score: nasce sulla base di un semplice strumento di controllo delle funzioni vitali, il cosiddetto early warning score (EWS), da cui derivare un valore numerico indicativo del rischio di cascata di eventi avversi. Il principio di base di EWS era quello di raccogliere parametri fisici di usuale e facile reperimento, riunendoli in una scala che consentisse al personale sanitario una veloce e condivisa valutazione dello stato clinico. Nei pazienti ricoverati, può consentire di cogliere il deterioramento delle funzioni fisiologiche, di definire l'intensità di cura necessaria, e fornire indicazioni precise per la frequenza e la tipologia dell'assistenza. La versione modificata, denominata MEWS, si compone di 5 macro-criteri (Figura 2) e definisce uno stato di instabilità clinica laddove venga raggiunto un punteggio $>5 .{ }^{33}$ Tale condizione si è dimostrata correlata ad elevato rischio di mortalità e di trasferimento in Unità di Terapia Intensiva. La validità dello score MEWS come predittore di outcome sfavorevole intra-ospedaliero è stata documentata anche nel contesto specifico delle Unità di Medicina Interna. ${ }^{34}$

iv) Vitalpac ${ }^{T M}$ early warning score (ViEWS): versione modificata del MEWS che considera fra i macrocriteri anche il valore di saturazione dell'ossigeno e un eventuale utilizzo supplementare di ossigeno. ${ }^{35}$

v) National early warning score (NEWS): una commissione del Royal College of Physicians ha redatto tale strumento, valido in tutti i servizi assistenziali. Il punteggio NEWS che ne deriva fornisce uno standard di riferimento universale per la valutazione del decorso clinico, con le sole eccezioni della casistica pediatrica e ostetrica e delle cure di fine vita. Il NEWS, come tutti i sistemi EWS, si fonda su 6 misurazioni di parametri fisiologici normalmente effettuate, a ciascuna delle quali è attribuito un valore numerico. La somma dei valori numerici fornisce la misura dello scostamento dalla condizione fisio-

Tabella 4. Principali strumenti per la valutazione della fragilità.

\begin{tabular}{|c|c|}
\hline Indice & Bibliografia \\
\hline Indice di Flugelman & Flugelman et al., $1986^{23}$ \\
\hline CHS frailty index & Fried et al., $2001^{24}$ \\
\hline Indice di Gill & Gill et al., $2002^{25}$ \\
\hline SOF frailty index & Ensrud et al., $2008^{26}$ \\
\hline$S H A R E-F I$ & Romero-Ortuno et al., $2010^{27}$ \\
\hline Indice HARP (hospital admission risk profile) & Sager et al., $1996^{28}$ \\
\hline Frailty index-comprehensive geriatric assessment (FI-CGA) & Jones et al., $2004^{29}$ \\
\hline
\end{tabular}


logica. Lo score è maggiorato nei casi in cui sia necessaria l'ossigeno-terapia. ${ }^{36}$

\section{Dipendenza funzionale}

Il concetto di dipendenza funzionale interviene in quella sfera dell'anziano che tratta l'autonomia fisica, ovvero la capacità dell'anziano di far fronte alle esigenze quotidiane. È un tipo di autonomia che è interdipendente ad altri due concetti: autonomia relazionale (deficit sensoriale, deficit cognitivi, deficit comunicativi) e autonomia sociale (risorse economiche, risorse organizzative, contesto socio-familiare). Gli anziani sono per lo più affetti da un alto grado di comorbidità, che provoca sovente nel lungo o nel breve termine uno stato di significativa disabilità. ${ }^{37} \mathrm{Tra}$ gli obiettivi della Medicina vi è proprio la necessità di prevenire la manifestazione nell'anziano di uno stato di dipendenza funzionale, cercando di garantire un miglioramento e/o mantenimento delle capacità funzionali del paziente e indirettamente assicurandone un sufficiente grado di quality of life. Diversi studi indicano l'ospedalizzazione come fattore di rischio nella perdita di autonomia funzionale e peggioramento della qualità di vita ${ }^{38}$ con importanti ripercussioni in termini di possibili ri-ospedalizzazioni, bisogni assistenziali e mortalità. ${ }^{39}$

Trasformare in numeri le abilità dei pazienti permette la schematizzazione, la trasferibilità e la confrontabilità dei dati nel tempo. La letteratura si è molto spesa al riguardo proponendo una serie di scale in grado di dare una valutazione alla dipendenza funzionale principalmente sulla base di 3 criteri: i) le attività di base: sovrapponibili ai bisogni di base (scala ADL e indice di Barthel); ii) le attività strumentali: presuppongono l'utilizzo di alcune tecnologie semplici oltre alla capacità di muoversi correttamente all'interno di un contesto sociale (scala IADL); iii) attività avanzate: attività che incidono sulla qualità della vita.

\section{Scala activity daily living}

Strumento di semplice utilizzo che valuta le capacità funzionali del paziente, senza supervisione o aiuto da parte di altri (tranne se specificato), nell'esecuzione di 6 attività della vita quotidiana: fare il bagno nella vasca o nella doccia, vestirsi, andare alla toilette, mobilità, con-

Modified Early Warning Score (MEWS)

\begin{tabular}{|c|c|c|c|c|c|c|c|c|}
\hline \multirow{2}{*}{$\begin{array}{l}\text { Paziente: } \\
\text { Reparto: }\end{array}$} & \multicolumn{2}{|c|}{ Data (gg/mm/aa) } & & & & & & \\
\hline & \multicolumn{2}{|c|}{ Ora (hh:mm) } & & & & & & \\
\hline \multirow{4}{*}{ Coscienza } & \multicolumn{2}{|l|}{ - (A) Sveglio } & 0 & 0 & 0 & 0 & 0 & 0 \\
\hline & \multicolumn{2}{|l|}{-(V) Risvegliabile alla chiamata } & 1 & 1 & 1 & 1 & 1 & 1 \\
\hline & \multicolumn{2}{|l|}{ - (P) Reagisce solo al dolore } & 2 & 2 & 2 & 2 & 2 & 2 \\
\hline & \multicolumn{2}{|c|}{ - (U) Non risponde alla chiamata e non reagisce al dolore } & 3 & 3 & 3 & 3 & 3 & 3 \\
\hline \multirow{5}{*}{ Respiro } & \multirow{5}{*}{ - Frequenza respiratoria (atti/min) } & $\geq 30$ & 3 & 3 & 3 & 3 & 3 & 3 \\
\hline & & $21-29$ & 2 & 2 & 2 & 2 & 2 & 2 \\
\hline & & $15-20$ & 1 & 1 & 1 & 1 & 1 & 1 \\
\hline & & $9-14$ & 0 & 0 & 0 & 0 & 0 & 0 \\
\hline & & $<9$ & 2 & 2 & 2 & 2 & 2 & 2 \\
\hline \multirow{11}{*}{ Circolo } & \multirow{6}{*}{ - Frequenza cardiaca (bpm) } & $\geq 130$ & 3 & 3 & 3 & 3 & 3 & 3 \\
\hline & & $111-129$ & 2 & 2 & 2 & 2 & 2 & 2 \\
\hline & & $101-110$ & 1 & 1 & 1 & 1 & 1 & 1 \\
\hline & & $51-100$ & 0 & 0 & 0 & 0 & 0 & 0 \\
\hline & & $41-50$ & 1 & 1 & 1 & 1 & 1 & 1 \\
\hline & & $\leq 40$ & 2 & 2 & 2 & 2 & 2 & 2 \\
\hline & \multirow{5}{*}{ - Pressione sistolica (mmHg) } & $\geq 200$ & 2 & 2 & 2 & 2 & 2 & 2 \\
\hline & & $101-199$ & 0 & 0 & 0 & 0 & 0 & 0 \\
\hline & & $81-100$ & 1 & 1 & 1 & 1 & 1 & 1 \\
\hline & & $71-80$ & 2 & 2 & 2 & 2 & 2 & 2 \\
\hline & & $\leq 70$ & 3 & 3 & 3 & 3 & 3 & 3 \\
\hline \multirow{3}{*}{ Febbre } & \multirow{4}{*}{ - Temperatura corporea $\left({ }^{\circ} \mathrm{C}\right)$} & $\geq 38.5$ & 2 & 2 & 2 & 2 & 2 & 2 \\
\hline & & $35-38.4$ & 0 & 0 & 0 & 0 & 0 & 0 \\
\hline & & $\leq 35$ & 2 & 2 & 2 & 2 & 2 & 2 \\
\hline & & TOTALE & & & & & & \\
\hline
\end{tabular}

Figura 2. Il modified early warning score (MEWS) (versione modificata). 
tinenza e capacità di alimentarsi. ${ }^{40}$ Generalmente vi è una perdita dalla prima all'ultima attività, mentre il recupero delle abilità avviene dall'ultima verso la prima.

Punteggio: 0 corrisponde alla completa dipendenza; 3 necessita di assistenza parziale; 6 corrisponde alla totale autosufficienza.

\section{Indice di Barthel}

Valuta 10 abilità: mangiare, spostarsi dalla sedia al letto e ritornare, toilette personale, sedersi e alzarsi dal wc, fare il bagno, camminare in piano o spingere la sedia a rotelle, salire e scendere le scale, vestirsi, controllo della defecazione, controllo della minzione. Il punteggio assegnato per ogni funzione può essere 15, 10,5 o 0 . Il punteggio massimo è assegnato solo se il paziente esegue il compito in modo completamente indipendente, senza la presenza di personale d'assistenza. Il punteggio massimo è 100, ed indica l'indipendenza in tutte le attività di base della vita quotidiana. ${ }^{41}$

\section{Scala IADL - Lawton e Brody}

È una scala che analizza il grado di autosufficienza di un soggetto in relazione a 8 attività strumentali della vita quotidiana necessarie per vivere autonomamente al proprio domicilio: capacità di usare il telefono, fare acquisti, preparazione del cibo, governo della casa, biancheria, mezzi di trasporto, responsabilità nell'uso di medicinali e capacità di maneggiare il denaro. ${ }^{42}$

Nel definire il punteggio si ricorre a una scala semplificata che prevede l'assegnazione di un punto per ciascuna funzione indipendente così da ottenere un risultato totale di performance che varia da 0 (completa dipendenza) a 8 (indipendenza in tutte le funzioni). Nei maschi, data la minore abitudine ad eseguire alcune compiti domestici, il punteggio massimo può essere limitato a 5 .

\section{Disfunzione cognitiva e depressione}

La depressione e la demenza hanno un'elevata prevalenza nella popolazione geriatrica e sono causa di grave disabilità in quanto vanno a condizionare quella sfera della autonomia a cui si faceva riferimento nel paragrafo precedente e definita come autonomia relazionale. Per entrambe le patologie, l'utilizzo di test specifici (Tabelle 5 e 6 ) è utile per cercare di porre una diagnosi precoce che consenta di instaurare un intervento farmacologico, comportamentale e assistenziale in grado di rallentare la progressione dei sintomi. Sono stati sviluppati e validati diversi test per entrambi questi domini.

\section{Scale di valutazione del deterioramento cognitivo}

Le scale di valutazione del deterioramento cognitivo sono riassunte in Tabella 5. ${ }^{43-46}$

\section{Short portable mental status questionnaire}

Lo short portable mental state questionnaire (SPMSQ) fu messo a punto con l'intento di fornire al clinico uno strumento semplice, maneggevole, di rapido e facile impiego, e al tempo stesso valido ed affidabile, per valutare la presenza ed il grado di compromissione della funzione cognitiva ${ }^{45}$ L'Autore ha focalizzato la sua attenzione sulla memoria a breve e a lungo termine, sull'orientamento, sull'informazione relativa ad eventi attuali e sulla capacità di eseguire semplici calcoli matematici. Lo SPMSQ è senz'altro uno strumento breve e maneggevole, non presenta particolari difficoltà di valutazione ed include l'intero range di performance intellettuali, dal funzionamento intellettivo normale alla compromissione più grave (Tabella 6 ).

\section{Scale di valutazione dell'umore}

La depressione è uno dei disturbi più comuni che affliggono le persone anziane, e si associa ad un aumento significativo della morbilità e della mortalità. Le più utilizzate modalità di valutazione dello stato dell'umore sono di seguito riportate (Tabella 7) ${ }^{47-51}$

\section{Geriatric depression scale}

La geriatric depression scale (GDS) è stata espressamente messa a punto per i pazienti anziani. ${ }^{50} \grave{E}$ stata inizialmente convalidata in un formato da 30 do-

Tabella 5. Scale di valutazione del deterioramento cognitivo.

\begin{tabular}{ll}
\hline Indice & Bibliografia \\
\hline $\begin{array}{l}\text { Blessed dementia scale (BDS) } \\
\text { Mini-mental state examination (MMSE) }\end{array}$ & Blessed et al.,1968 \\
\hline $\begin{array}{l}\text { Short portable mental status questionnaire (SPMSQ) } \\
\text { Philadelphia geriatric center mental status questionnaire } \text { et al., } 1975^{44}\end{array}$ & Pfeiffer et al., 1975 \\
\hline $\begin{array}{l}\text { Questi test vengono somministrati in circa 10 minuti e possiedono una elevata test-retest reliability. [La test-retest reliability valuta l'affidabilità di un test verificando } \\
\text { la stabilità dello strumento in somministrazioni successive; ad esempio, somministriamo lo stesso test a distanza di } 3 \text { mesi e andiamo ad accertare in che misura i } \\
\text { punteggi ottenuti la prima volta sono correlati con quelli rilevati la seconda volta. Maggiore sarà la concordanza tra i due punteggi, più elevato risulterà il coefficiente } \\
\text { di correlazione (coefficiente di attendibilità test-retest)]. }\end{array}$
\end{tabular}


mande, dopo di che sono state proposte anche versioni da 15 domande $^{52}$ e 5 domande. ${ }^{53}$ I punteggi limite per un esame della depressione positivo sono rispettivamente 11,7 e 2 (Tabella 8).

\section{Nutrizione}

Un alterato stato di nutrizione condiziona il processo di invecchiamento e lo stato di salute degli individui in età senile. ${ }^{54}$ La prevalenza di malnutrizione tra i pazienti anziani ospedalizzati risulta molto variabile e comunque elevata, con valori che oscillano tra il 30 e il $60 \%$ di casi. ${ }^{55}$ L'avanzare dell'età comporta modifiche dei processi di adattamento fisiologico che portano ad alterazioni nell'assunzione dei nutrienti. ${ }^{56}$

La malnutrizione risulta un fattore di rischio indipendente di morbilità e di ri-ospedalizzazione, ${ }^{57} \mathrm{ma}$ anche la mortalità durante il ricovero risulta correlata a variabili antropometriche, fra cui le maggiormente predittive sono risultate essere albumina e peso corporeo. ${ }^{58}$

La malnutrizione si correla inoltre ad un peggioramento di patologie croniche, maggiore incidenza di infezioni, comparsa di piaghe da decubito, maggior rischio di fratture, depressione ed apatia, peggioramento della qualità di vita.

Per una accurata valutazione dello stato nutrizionale è possibile considerare diverse tecniche: clinica, biumorale, strumentale, multidimensionale.

Tra gli strumenti multidimensionali per la valutazione dello stato nutrizionale, sono state proposte tabelle o questionari, tra questi:
- Mini nutritional assessment (MNA): L'MNA fornisce un metodo semplice e rapido per individuare i pazienti anziani malnutriti o a rischio di malnutrizione prima che subentrino gravi alterazioni ponderali o della proteinemia. Comprende 18 items suddivisi in 3 settori principali (antropometria e variazioni ponderali, valutazione dell'introito alimentare qualitativo e quantitativo, stato di disabilità e cognitivo) e punteggio massimo 30 . Un punteggio inferiore a 17 è indicativo di malnutrizione per difetto, un punteggio compreso tra $17 \mathrm{e}$

Tabella 6. Short portable mental status questionnaire.

\begin{tabular}{l}
\hline 1. Qual è la data di oggi (giorno, mese, anno)? \\
\hline 2. Che giorno della settimana? \\
\hline 3. Qual è il nome di questo posto? \\
\hline 4. Qual è il suo numero di telefono? \\
\hline 4a. Qual è il suo indirizzo? \\
\hline 5. Quanti anni ha? \\
\hline 6. Quando è nato? \\
\hline 7. Chi è il Presidente della Repubblica? \\
\hline 8. Chi era il Presidente precedentemente? \\
\hline 9. Quale era il cognome di sua madre da ragazza? \\
\hline 10. Sottragga 3 da 20 e da ogni numero successivo fino in fondo \\
\hline 0-2 errori=Integrità intellettiva; \\
5-4 errori=Disfunzione cognitiva leggera; \\
8-10 errori=Disfunzione cognitiva moderata;
\end{tabular}

Tabella 7. Scale di valutazione dell'umore.

\begin{tabular}{ll}
\hline Indice & Bibliografia \\
\hline Measurement of morale in the elderly - MME & Pierce et al., $1973^{47}$ \\
\hline Philadelphia geriatric center morale scale - PGCMS & Lawton et al., $1975^{48}$ \\
\hline Short psychiatric evaluation schedule - SPES & Pfeiffer et al., $1979^{49}$ \\
\hline Geriatric depression scale - GDS & Yesavage et al., 1982 \\
\hline Cornell scale for depression in dementia - CSDD & Alexopoulos et al., $1988^{51}$ \\
\hline
\end{tabular}

Tabella 8. 5-item geriatric depression scale.

\begin{tabular}{lcc}
\hline & Sì No & \multicolumn{1}{c}{} \\
\hline È soddisfatto della sua vita? & 0 \\
\cline { 1 - 1 } È di buon umore per la maggior parte del tempo? & 0 \\
\hline Si sente spesso indifeso, abbandonato? & 1 & 0 \\
\hline Preferisce stare a casa piuttosto che uscire a fare cose nuove? & 1 & 0 \\
\hline Si sente un po' inutile per come lei vive in questo periodo? & 1 & 0 \\
\hline
\end{tabular}


23,5 è indicativo di rischio di malnutrizione, un punteggio superiore a 24 indica uno stato nutrizionale nella norma. ${ }^{59}$

- Malnutrition universal screening tool (MUST): tra i molteplici indici nutrizionali disponibili, il MUST consente di identificare lo stato nutrizionale in soggetti adulti in modo semplice, rapido e riproducibile. Esso rappresenta uno dei tanti indici nutrizionali integrati che, considerando determinati parametri individuali (ad esempio il sottopeso, l'entità del decremento ponderale, le patologie presenti), giungono a calcolare un punteggio totale; quest'ultimo, se superiore a determinati valori soglia, indica la presenza di un rischio lieve, moderato o grave di malnutrizione. ${ }^{60}$

- Valutazione soggettiva dello stato nutrizionale (SGA): tale valutazione prende in considerazione vari domini: la storia ponderale; una valutazione dei cambiamenti dell'introito alimentare; un'indagine su alcuni sintomi gastroenterici; una valutazione della capacità funzionale basata sull'attività motoria; una valutazione della presenza di patologie concomitanti; un esame soggettivo dello stato nutrizionale (ipotrofia muscolare, riduzione del pannicolo adiposo, edema, ascite). ${ }^{61}$

- NRS-2002 - nutritional risk screening: proposto dalla Società Europea di Nutrizione Enterale e Parenterale, lo strumento è stato validato per il paziente ospedalizzato. ${ }^{62}$ Il questionario consta di 4 semplici domande: se tutte risultano negative, il paziente non è definito a rischio, se anche solo una delle risposte risulta positiva, si procede allo screening finale secondo punteggi prestabiliti.

\section{Conclusioni}

Una più appropriata definizione del profilo di complessità e quindi di rischio di outcome sfavorevole del paziente ricoverato, può sensibilmente contribuire a un miglioramento della qualità dell'assistenza intraospedaliera, indirizzandola verso livelli di intensità individualizzati. Contemporaneamente, essa può generare informazioni utili a gestire la continuità assistenziale nella delicata transizione ospedale-territorio, ottimizzando le potenzialità dei sistemi sanitari dedicati alla post-acuzie e alla cronicità.

La gestione del paziente complesso richiede un approccio che vada al di là del semplice coordinamento delle varie prestazioni specialistiche: è opportuno che essa si configuri come messa a punto di percorsi diagnostici-terapeutici-riabilitativi il più possibile individualizzati, con buon rapporto costo/efficacia, e che prevedano sempre di più l'empowerment del paziente e della sua famiglia, e la costituzione di percorsi assistenziali in continuità ospedale-territorio. Presupposto fondamentale è in ogni caso la disponibilità di stru- menti accuratamente sviluppati e validati, che sappiano esprimere in modo il più possibile inclusivo i molteplici domini che contribuiscono a definire la complessità individuale, e che siano soprattutto di agevole gestione per poter essere efficacemente e sistematicamente integrati nella pratica clinica. Lo studio COMPLIMED che FADOI sta conducendo, considera non soli i domini sopra-descritti, ma altri aspetti rilevanti del paziente complesso ospedalizzato quali il rischio di cadute, il delirium, l'aderenza alla terapia, la valutazione del dolore, la presenza di eventuali ulcere da pressione, la condizione familiare, le politerapie. Vogliamo verificare se dallo studio COMPLIMED potremo ricavare uno strumento di semplice utilizzo in grado di indirizzare al meglio la gestione del paziente complesso.

\section{Bibliografia}

1. United Nations. Population ageing and development 2012. New York, NY: United Nations; 2012. Available from: http://www.un.org/esa/population/publications/ 2012WorldPopAgeingDev_Chart/2012PopAgeingandDev_WallChart.pdf Accessed: 26 August 2013.

2. Nardi R, Scanelli G, Borioni D, et al. The assessment of the complexity in internal medicine patients. The FADOI Medicomplex Study. Eur J Int Med 2007; 18:283-7.

3. Nardi R, Scanelli G, Corrao S, et al. Comorbidity does not reflect complexity in internal medicine patients. Eur J Int Med 2007;18:359-68.

4. I Quaderni del Ministero della Salute. Criteri di appropriatezza clinica, tecnologica e strutturale nella assistenza del paziente complesso, N. 23, Settembre-Ottobre 2013.

5. Gensini GF, Fabbri LM, Fini M, Nozzoli C (a cura di). La Medicina della complessità: BPCO e comorbidità. Firenze: Firenze University Press; 2010.

6. Turner BJ, Cuttler L. The complexity of measuring clinical complexity. Ann Intern Med 2011;155:851-2.

7. Bernabeu-Wittel M, Ollero-Baturone M, Moreno-Gavino L, et al. Development of a new predictive model for polypathological patients. The PROFUND Index. Eur J Intern Med 2011;22:311-7.

8. Pilotto A, Ferrucci L, Franceschi M, et al. Development and validation of a multidimensional prognostic index for-one year mortality from comprehensive geriatric assessment in hospitalized older patients. Rejuvenation Res 2008;11:151-61.

9. Ministero della Salute. Relazione sullo stato sanitario del Paese 2009-2010. Roma: Ministero della Salute; 2010. Available from: http://www.rssp.salute.gov.it/rssp/ documenti/RSSP_2009_2010.pdf

10. Zulman DM, Asch SM, Martins SB, et al. Quality of care for patients with multiple chronic conditions: the role of comorbidity interrelatedness. J Gen Intern Med 2014;29:529-37.

11. Parmalee PA, Thuras PD, Katz IR, Lawton MP. Validation of the cumulative illness rating scale in a geriatric residential population. J Am Geriatr Soc 1995;43:130-7.

12. Charlson ME, Szatrowski TP, Peterson J, Gold J. Validation of a combined comorbidity index. J Clin Epidemiol 1994;47:1245-51. 
13. Greenfield S, Sullivan L, Dukes KA, et al. Development and testing of a new measure of case mix for use in office practice. Med Care 1995;33:AS47-55.

14. Kaplan M, Fenstein A. The importance of classifying initial co-morbidity in evaluating the outcome of diabetes mellitus. J Chron Dis 1974;27:387-404.

15. Holtzman J, Lurie N. Causes of increasing mortality in a nursing home population. J Am Geriatr Soc 1996; 44:258-64.

16. McCormick WC, Kukull WA, van Belle G, et al. Symptom patterns and comorbidity in the early stages of Alzheimer's disease. J Am Geriatr Soc 1994;42:517-21.

17. Jaeger AA, Hlatky MA, Paul SM, Gortner SR. Functional capacity after cardiac surgery in elderly patients. J Am Coll Cardiol 1994;24:104-8.

18. Fisher AL. Just what defines frailty? JAGS 2005;53: 2229-30.

19. Fried LP, Ferrucci L, Darer J, et al. Untangling the concepts of disability, frailty, and comorbidity: implications for improved targeting and care. J Gerontol A Biol Sci Med Sci 2004;59:255-63.

20. Gobbens RJ, Luijkx KG, Wijnen-Sponselee MT, Schols JM. In search of an integral conceptual definition of frailty: opinions of experts. J Am Med Dir Assoc 2010;11:338-43.

21. Rockwood K, Andrew M, Mitnitski A. A comparison of two approaches to measuring frailty in elderly people. J Gerontol A Biol Sci Med Sci 2007;62:738-43.

22. Hogan DB, MacKnight C, Bergman H. Models, definitions, and criteria of frailty. Aging Clin Exp Res 2003;15:1-29.

23. Flugelman MY, Ben David Y, Harats N, Eliakim M. A simple prognostic index for hospitalized geriatric patients. Gerontology 1986;32:272-6.

24. Fried LP, Tangen CM, Walston J, et al. Frailty in older adults: evidence for a phenotype. J Gerontol A Biol Sci Med Sci 2001;56:M146-57.

25. Gill TM, Baker DI, Gottschalk M, et al. A program to prevent functional decline in physically frail, elderly persons who live at home. N Engl J Med 2002;347: 1068-74.

26. Ensrud KE, Ewing SK, Taylor BC, et al. Comparison of 2 frailty indexes for prediction of falls, disability, fractures, and death in older women. Arch Intern Med 2008;168:382-9.

27. Romero-Ortuno R, Walsh CD, Lawlor BA, Kenny RA. A frailty instrument for primary care: findings from the Survey of Health, Ageing and Retirement in Europe (SHARE). BMC Geriatr 2010;10:57.

28. Sager MA, Rudberg MA, Jalaluddin M, et al. Hospital admission risk profile (HARP): identifying older patients at risk for functional decline following acute medical illness and hospitalization. J Am Geriatr Soc 1996;44:251-7.

29. Jones DM, Song X, Rockwood K. Operationalizing a frailty index from a standardized comprehensive geriatric assessment. J Am Geriatr Soc 2004;52:1929-33.

30. Bozzano C, Lancini I, Mei E, et al. L'indice di Flugelman per individuare pazienti complessi e di difficile dimissione. Ital J Med 2011;5:103-8.

31. Halm EA, Fine MJ, Kapoor WN, et al. Instability on hospital discharge and the risk of adverse outcomes in patients with pneumonia. Arch Intern Med 2002;162: 1278-84
32. Bernardini B, Cappadonia C, Giardini S, et al. Sistema IPER indici di processo- Esito in riabilitazione. Mareno di Piave (TV): Edizioni Vega; 2004. pp 87-91.

33. Subbe CP, Kruger M, Gemmel L. Validation of a modified early warning score in medical admissions. Quarterly J Med 2001;94;521-6.

34. Cei M, Bartolomei C, Mumoli N. In-hospital mortality and morbidity of elderly medical patients can be predicted at admission by the modified early warning score: a prospective study. Int J Clin Pract CME 2009;63:591-5.

35. Kellett J, Wang F, Woodworth S, Huang W. Changes and their prognostic implications in the abbreviated VitalPAC ${ }^{\text {TM }}$ Early Warning Score (ViEWS) after admission to hospital of 18,827 surgical patients? Resuscitation 2013;84:471-6.

36. McGinley A, Pearse RM. A national early warning score for acutely ill patients. BMJ 2012;345:e5310

37. Verbrugge LM, Jet-I-E A. The disablement process. Sm Sci Med 1994;38:1-14.

38. Covinsky KE, Palmer RM, Fortinsky RH, et al. Loss of independence in activities of daily living in older adults hospitalized with medical illnesses: increased vulnerability with age. J Am Geriatr Soc 2003;51:451-8.

39. Fortinsky RH, Covinsky KE, Palmer RM, Landefeld $\mathrm{CS}$. Effects of functional status changes before and during hospitalization on nursing home admission of older adults. J Gerontol A Biol Sci Med Sci 1999;54:M521-6.

40. Katz S. Assessing self-maintenance: activities of daily living, mobility, and instrumental activities of daily living. J Am Geriatric Soc 1983;31:721-7.

41. Barthel DW, Mahoney FI. Functional evaluation: the Barthel index. Maryland State Med J 1965;14:61-5.

42. Lawton MP, Brody EM. Assessment of older people: self-maintaining and instrumental activities of daily living. Gerontologist 1969;9:179-86.

43. Blessed G, Tomlinson BE, Roth M. The association between quantitative measures of dementia and of senile change in the cerebral grey matter of elderly subjects. Br J Psychiatry 1968;114:797-811.

44. Folstein MF, Folstein SE, McHugh PR. Mini-mental state. A practical method for grading the cognitive state of patients for the clinician. J Psychiatr Res 1975;12: 189-98.

45. Pfeiffer E. A short portable mental status questionnaire for the assessment of organic brain deficit in elderly patients. J Am Geriatrics Soc 1975;23:433-41.

46. Fishback DB. Mental status questionnaire for organic brain syndrome, with a new visual counting test. J Am Geriatr Soc 1977;25:167-70.

47. Pierce RC, Clark MM. Measurement of morale in the elderly. Int J Aging Hum Dev 1973;4:83-101.

48. Lawton MP. The Philadelphia Geriatric Center Morale Scale: a revision. J Gerontol 1975;30:85-9.

49. Pfeiffer E. A short psychiatric evaluation schedule: a new 15-item monotonic scale indicative of functional psychiatric disorder. Brain Funct Old Age Bayer-Symp 1979;7:228-36.

50. Yesavage JA, Brink TL, Rose TL, et al. Development and validation of a geriatric depression screening scale: a preliminary report. J Psychiatr Res 1982-1983;17:37-49.

51. Alexopoulos GS, Abrams RC, Young RC, Shamoian CA. Cornell Scale for Depression in Dementia. Biol Psychiatry 1988;23:271-84. 
52. Sheikh JI, Yesavage JA. Geriatric depression scale (GDS): recent evidence and development of a shorter version. Clinical Gerontology: a guide to assessment and intervention 165-173. New York, NY: The Haworth Press; 1986.

53. Hoyl MT, Alessi CA, Harker JO, et al. Development and testing of a five-item version of the geriatric depression scale. J Am Geriatr Soc 1999;47:873-8.

54. Solomons NW. Demographic and nutritional trends among the elderly in developed and developing regions. Eur J Clin Nutr 2000;54:S2-14.

55. Keller HH. Malnutrition in istitutionalized elderly: how and why? J Am Geriatr Soc 1993;41:1212-8.

56. Morley JE, Mooradian AD, Silver AJ, et al. Nutrition in the elderly. Ann Intern Med 1988;109:890-904.

57. Sullivan DH. Risk factors for early hospital readmission in a select population of geriatric rehabilitation patients: the significance of nutritional status. J Am Geriatr Soc 1992;40:792-8.
58. Sullivan DH, Patch GA, Walls RC, Lipschitz DA. Impact of nutrition status on morbidity and mortality in a select population of geriatric rehabilitation patients. Am J Clin Nutr 1990;51:749-58.

59. Guigoz Y, Vellas B, Garry PJ. Assessing the nutritional status of the elderly: the mini nutritional assessment as part of the geriatric evaluation. Nutr Rev 1996;54;S59-65.

60. Malnutrition Advisory Group. The "MUST" report: nutritional screening for adults. A multidisciplinary responsibility. Redditch, Worcestershire: MAG; 2003.

61. Goldstein J. Assessment of nutrition status in renal disease. In: Mitch WE, Klahr S, eds. Handbook of nutrition and the kidney. Philadephia: Lippincot-Raven; 1998. pp 45-86.

62. Kondrup J, Rasmussen HH, Hamberg O, Stanga Z. Nutritional risk screening (NRS 2002): a new method based on an analysis of controlled clinical trials. Clin Nutr 2003;22:321-36. 


\title{
La stratificazione per severità dei pazienti ricoverati in Medicina Interna: un lavoro ancora incompiuto. Valutazione clinica e non strumenti surrogati
}

\author{
Giuseppe Chesi, ${ }^{1}$ Roberto Nardi ${ }^{2}$ \\ ${ }^{1}$ Dipartimento Internistico Area Sud Montana, Ospedale Magati di Scandiano (RE); ${ }^{2}$ Medicina Interna, Bologna, Italia
}

\begin{abstract}
RIASSUNTO
Nonostante la molteplicità degli strumenti disponibili in Letteratura sui metodi di valutazione dei pazienti con malattie critiche, pur riconoscendo il valore di tali mezzi, ribadiamo l'importanza del giudizio clinico globale nella stratificazione prognostica e nell'allocazione più appropriata dei pazienti critici complessi in Medicina Interna. Gli scores possono essere più utilmente impiegati per il monitoraggio seriato infermieristico di tali pazienti. La loro lettura ed interpretazione non può prescindere dalla valutazione clinica complessiva di ogni singolo malato da parte del medico.
\end{abstract}

\section{Introduzione}

Nella riorganizzazione del sistema sanitario in corso il modello assistenziale per intensità di cure, pur ancora non perfettamente definito, sta progressivamente prendendo piede, secondo una strategia finalizzata all'allocazione dei pazienti in setting di accoglienza adeguati alle esigenze della complessità clinica e dei bisogni assistenziali degli stessi. Purtroppo esiste ancora grande confusione nell'impiego dei termini impiegati, sia nella definizione di complessità assistenziale che di intensità di cura (inteso come livello di cura richiesto dal caso, conseguente ad una valutazione dell'instabilità clinica e della complessità dei bisogni medici ed infermieristici) (Tabella 1), nonché nella definizione di paziente complesso, critico, instabile e fragile. ${ }^{1-3}$

Corrispondente: Giuseppe Chesi, Dipartimento di Medicina Interna, Ospedale di Scandiano, Azienda USL Reggio Emilia, Italia. E-mail: chesig@ausl.re.it

Parole chiave: valutazione del paziente critico complesso, MEWS, VIEWS, NEWS.

Ringraziamenti: si ringrazia la dott.ssa S. Nardi per la preziosa collaborazione fornita nella revisione italiana dell'articolo originale.

Modificato da: G. Chesi, R. Nardi. Severity stratification of patients hospitalized on the Internal Medicine ward: work in progress. Ital J Med 2013;7:231-3.

Articolo pubblicato secondo la Creative Commons Attribution NonCommercial 3.0 License (CC BY-NC 3.0).

(C) Copyright G. Chesi e R. Nardi, 2014

Licensee PAGEPress, Italy

QUADERNI - Italian Journal of Medicine 2014; 2:25-28
I diversi ambiti della complessità clinica, infermieristica e gestionale dei pazienti ricoverati in medicina interna richiedono un approccio multidisciplinare ed una valutazione multidimensionale (Figura 1).

La comunità scientifica negli ultimi anni si è interrogata riguardo alla possibilità di identificare strumenti attendibili in grado di stratificare la severità dei pazienti ammessi in ospedale, anche per una loro diversa allocazione in base alla differenziazione dei livelli di cura adeguati e necessari per il loro grado di malattia. Parallelamente, partendo dalla considerazione che un certo numero di pazienti che si instabilizzavano nel corso di un ricovero ospedaliero non ricevevano tempestivamente cure adeguate, né venivano trasferiti in setting più appropriati, ${ }^{4} \mathrm{si}$ era cercato di validare scores di facile esecuzione anche da parte di personale non medico, al fine di migliorare il riconoscimento precoce di queste situazioni per facilitare un più tempestivo trasferimento in setting assistenziali più idonei, adeguati al grado di instabilità. Il più noto strumento proposto $\mathrm{e}$ variamente utilizzato a questi scopi è stato il modified early warning score (MEWS). ${ }^{5,6}$ In effetti il MEWS (con le sue successive modificazioni proposte) non è nato come score finalizzato alla valutazione della severità dei pazienti al momento dell'ammissione in unità di cure ospedaliere, ma come strumento di facile uso per il controllo seriato delle condizioni cliniche di pazienti degenti in aree di tipo generalistico, in cui l'assenza di strumenti tecnologici di monitoraggio continuo poteva ritardare eventuali interventi necessari in pazienti con rapido deterioramento clinico. ${ }^{7}$ Non sono molti invece i lavori che hanno considerato l'utilizzo del MEWS per la stratificazione dei pazienti ammessi in ospedale, sia in reparti di Medicina Interna che in altre strutture specialistiche. Nei pochi lavori riportati in studi retrospettivi la casistica veniva ripartita in classi di severità tramite il calcolo del MEWS solamente 
dopo che il paziente era già stato allocato all'interno della unità operativa. ${ }^{89}$ La fotografia che si ricava da questi lavori - tra l'altro piuttosto disomogenei tra di loro - è quella di una percentuale di pazienti ammessi in unità internistiche con punteggi elevati di MEWS piuttosto bassa, con prevalenza di classi di pazienti con score compreso tra 0 e 1 . Molti di questi sarebbero da considerare forse potenzialmente suscettibili di un'assistenza extraospedaliera e in questi il ricovero ospedaliero potrebbe essere definito come inappropriato. ${ }^{10}$ A confutazione di tale osservazione, sta il fatto che Il MEWS esplora l'instabilità clinica dei pazienti ma è assolutamente inadeguato a valutarne la complessità secondo le diverse dimensioni ed inoltre considera solamente parametri di base e nessun elemento clinico. ${ }^{1}$ Un'ulteriore criticità riportata in letteratura per il MEWS si riferisce alla frequenza respiratoria, alla quale solitamente il personale medico ed infermieristico dei reparti internistici non è abituato a prestare attenzione. ${ }^{11}$ Per questo motivo in letteratura diversi autori hanno proposto modifiche e/o integrazioni di questo score. Come parametro surrogato rispetto alla frequenza respiratoria, più facilmente rilevabile ed ormai ampiamente usato anche nei reparti internistici, è stata indicata la saturazione arteriosa in ossigeno, considerando anche in associazione la necessità $o$ meno di correzione con ossigenoterapia. ${ }^{12,13}$ Questo parametro tuttavia esplora in maniera molto più settoriale la funzione respiratoria e la ventilazione del paziente e non considera ad esempio il grado di riserva funzionale connesso al distress della muscolatura respiratoria. Lo score così ampliato a 6 parametri e ribattezzato national early warning score (NEWS) è stato proposto come strumento efficace di valutazione della instabi-

Tabella 1. Intensità di cura e complessità assistenziale.

\begin{tabular}{ll}
\hline Intensità di cura $^{2}$ & Complessità assistenziale $^{\mathbf{3}}$ \\
\hline Assegnare al malato il posto letto collocato nel settore più appropriato & L'insieme delle prestazioni mediche ed infermieristiche riferite alle \\
rispetto ai suoi bisogni assistenziali, legati non solo alla tipologia & diverse dimensioni dell'assistenza, in termini di intensità di impegno, \\
di ricovero, ma anche alla sua condizione clinica e di dipendenza & di tempo e quantità e/o qualità di lavoro dei professionisti coinvolti \\
\hline
\end{tabular}

\section{Paziente complesso COMPLESSITA' CLINICA}

- severità di malattia

- instabilità clinica

- quadro clinico atipico

- difficoltà diagnostica,

- malattia rara

- multimorbilità attiva

- disfunzione cognitiva

- depressione

- fragilità biologica

- alto rischio di ADR/ACE

- eventi a cascata, complicanze

- failure to thrive e s. correlate $\left(^{*}\right)$

- procedure complesse

- terapie complesse

- bisogno di recupero funzionale

- necessità di follow-up

- monitoraggio

- gestione devices e strumenti

ADR/ACE: adverse drug events/adverse clinical events

$\left(^{\star}\right)$ ridotta autonomia funzionale, malnutrizione, depressione, disfunzione cognitiva, cadute, delirium ecc.

Figura 1. I domini della complessità nel paziente ricoverato in Medicina Interna.
Paziente complesso

COMPLESSITA' INFERMIERISTICA

- respirazione

- alimentazione e idratazione

- eliminazione urinaria e

fecale

- igiene

- movimento

- riposo e sonno

- funzione cardiocircolatoria

- ambiente sicuro

- interazione e

comunicazione

- medicazioni avanzate

- procedure terapeutiche

- procedure diagnostiche

- monitoraggio

- gestione devices e

strumenti ASSISTENZIALE

\section{Paziente complesso COMPLESSITA' GESTIONALE}

- ansia, paura

- scarsa compliance,

- collaborazione scarsa

- comunicazione difficile

- isolamento sociale

- solitudine, vedovanza

- rete sociale

- stress famigliare

- recente dimissione

- dimissione diffiicile

- frequent users

- dipendenza funzionale

- a rischio di caduta

- disfunzione cognitiva

- politerapia

- ${ }^{\circ}$ di health providers

- coordinamento delle cure

- decision making 
lizzazione di un paziente ricoverato dal Royal College of Physician Inglese ${ }^{4}$ e successivamente con il nome di Vitalpac ${ }^{T M}$ early warning score (ViEWS) e con solo lievi differenze nella modalità di utilizzo,${ }^{14}$ anche se i promotori del NEWS vi associavano una più fine e dettagliata analisi di corretto impiego, mettendo comunque in guardia le strutture da un suo utilizzo totalmente svincolato dalla clinica. Nonostante tali tentativi di miglioramento degli strumenti a disposizione, questi score presentano ancora alcuni importanti limiti intrinseci che, a nostro giudizio, se non adeguatamente considerati e corretti, possono inficiarne in maniera significativa l'utilizzo specie nella stratificazione di severità dei pazienti in ammissione. I due principali bias di utilizzo sono: i) il pesante condizionamento a cascata che la presenza di una iperpiressia significativa è in grado di determinare su quasi tutti gli altri parametri presenti, specialmente in un paziente di età avanzata; ii) la presenza di patologie potenzialmente in grado di instabilizzazioni acutissime, potenzialmente mortali in assenza al momento dell'ammissione di elementi clinici in grado di determinare lo sviluppo di un punteggio MEWS elevato. Per quanto riguarda il primo punto è rilievo comune l'osservare che un paziente febbrile, magari anche nel corso di una semplice febbre virale, può sviluppare tachicardia, polipnea, un calo della saturazione ed un ridotto livello di vigilanza che potrebbero comportare un punteggio MEWS anche di 3 o 4 punti. Parametri che una volta sfebbrato potrebbero rientrare tutti nella norma e comportare quindi un MEWS completamente diverso e più vicino allo 0 . Probabilmente con l'intento di superare questo problema, alcuni autori, nell'ambito dei dipartimenti di Emergenza Urgenza, hanno proposto uno score simile, che, a differenza di MEWS e ViEWS, non considera tra i parametri la temperatura. Tale score, denominato rapid emergency medicine score (REMS), ${ }^{15}$ comprende 6 item (età, frequenza cardiaca, frequenza respiratoria, pressione arteriosa media, Glasgow coma scale e saturazione arteriosa in $\mathrm{O}_{2}$ ) non ha ancora trovato molto seguito in letteratura. Se, da un lato, esso evita il confondimento determinato dalla temperatura elevata, dall'altro introduce il parametro età che, a motivo della ben nota variabilità dell'età biologica dei soggetti, può diventare un ulteriore fattore di confusione. Molte patologie sono ad elevato potenziale rischio di repentina instabilizzazione, ad esempio sindrome coronarica acuta, pancreatite acuta, un'emorragia digestiva, eccetera. Tutte queste situazioni possono presentare all'ingresso un MEWS, NEWS o ViEWS molto basso, pur essendo situazioni in grado di complicarsi rapidamente con aritmie maligne o stati di shock non altrimenti prevedibili. Per questi motivi per esse, specie nelle fasi iniziali, è richiesto un attento monitoraggio che evidentemente non può prescindere da un setting assistenziale adeguato, dotato anche di strumenti tecnologici, quali il monitoraggio poliparametrico ed altri ausili. ${ }^{16,17} \mathrm{Le}$ considerazioni conseguenti sono che probabilmente il più efficace strumento di valutazione dell'instabilizzazione dei pazienti è quello dell'osservazione clinica, da ripetersi più volte nell'arco della giornata. ${ }^{7}$ Il rilevante condizionamento da parte del parametro temperatura, la presenza di condizioni cliniche di per sé in grado di rapida e pericolosa instabilizzazione - indipendentemente dal punteggio di score MEWS o NEWS o VieWS all'ingresso - fanno sì che difficilmente almeno al momento questi strumenti possano tout court sostituirsi al giudizio clinico. ${ }^{18} \mathrm{E}$ questo è quello che viene anche ribadito dagli estensori di alcuni di questi scores, che, come nel caso del NEWS ${ }^{19}$ ribadiscono nelle loro conclusioni come esso non debba mai essere anteposto al giudizio clinico e come in alcune situazioni particolari, quali fine vita, demenza avanzata, allettamento cronico, etc., non debba essere applicato, onde evitare eccessi o accanimenti terapeutici. In letteratura a tutt'oggi non vi sono altre proposte né studi al riguardo: è necessario lavorare ancora e raccogliere ulteriori dati al fine di stabilire se e come effettivamente scores surrogati possano diventare un ausilio reale, a supporto del giudizio clinico e ad integrazione della diagnosi di ingresso nell'algoritmo decisionale, ai fini della migliore allocazione del paziente, in un sistema ospedaliero strutturato per intensità di cure. ${ }^{20,21}$ Per ora possiamo limitarci a dire che MEWS, NEWS e ViEWS possono essere utilmente impiegati come sistema di alert rapido in reparti internistici, particolarmente da parte del personale infermieristico, ma non possono mai sostituirsi in toto al giudizio clinico. ${ }^{12}$ Il dibattito ed i contributi pubblicati negli ultimi anni in letteratura al riguardo hanno avuto il merito di focalizzare l'attenzione della comunità medica sulla necessità di costruire strumenti semplici e facilmente applicabili in grado di evidenziare la severità e la criticità di un singolo paziente, nel contesto di cambiamenti organizzativo assistenziali finalizzati ad una distribuzione dell'assistenza basata sull'intensità di monitoraggio e di cure erogate. ${ }^{20}$ Sempre tuttavia da impiegare come completamento ed ausilio del giudizio clinico che, in una medicina sempre più complessa quale quella attuale rimane e rimarrà probabilmente comunque elemento essenziale. È questa una sfida per la medicina del futuro, nell'ambito della quale il medico internista per la sua visione olistica, la sua capacità di compendiare i problemi e selezionare le priorità, senza rinunciare alla tecnologia, potrà avere un ruolo sempre più importante. ${ }^{22}$

\section{Bibliografia}

1. Nardi R, Berti F, Greco A, et al. Complexity in hospital internal medicine departments: what are we talking about? Ital J Med 2013;7:142-55. 
2. Briani S, Cortesi E. L'ospedale per intensità di cure: aspetti teorici e problemi aperti. Igiene e Sanità Pubblica 2007;63:577-86

3. Moiset C, Vanzetta M. Misurare l'assistenza - il SIPI: dalla progettazione all'applicazione. Milano: Mc GrawHill; 2009.

4. Smith GB, Prytherch DR, Schmidt P, et al. Hospitalwide physiological surveillance - a new approach to the early identification and management of the sick patient. Resuscitation 2006;71:19-28.

5 Heitz CR, Gaillard JP, Blumstein H, et al. Performance of the maximum modified early warning score to predict the need for higher care utilization among admitted emergency department patients. J Hosp Med 2010;5: E46-52.

6. Burch VC, Tarr G, Morroni C. Modified early warning score predicts the need for hospital admission and in hospital mortality. Emerg Med J 2008;25:674-8.

7. Ludikhuize J, Smorenburg S, de Rooij S, de Jonge E. Identification of deteriorating patients on general wards; measurement of vital parameters and potential effectiveness of the Modified Early Warning Score. J Crit Care 2012;27:424.e7-13.

8. Bollini G, Colombo F. L'intensità assistenziale e la complessità clinica: un progetto di ricerca della Regione Lombardia. Milano: Era Futura; 2011. Available from: http://www.era-futura.com/wordpress/wp-content/ uploads/2012/02/Intensit\%C3\%A0-Assistenziale-e-laComplessit\%C3\%A0-Clinica.pdf

9. Robb G, Seddon M. A multi-faceted approach to the physiologically unstable patient. Qual Saf Health Care 2010;19:e47.

10. Joynt KE, Lha AK. Perspective path forward on medicare readmissions. New Engl J Med 2013;368:1175.

11. Ludikhuize J, de Jonge E, Goossens A. Measuring adherence among nurses one year after training in applying the modified early warning score and situation-background-assessment-recommendation instruments. Resuscitation 2011;82:1428-33.

12. Hammond NE, Spooner AG, Barnett AG, et al. The effect of implementing a modified early warning scoring (MEWS) system on the adequacy of vital sign documentation. Austr Crit Care 2013;26:18-22.
13. Kellett G, Woodworth S, Wang S, Huang W. Changes and their prognostic implications in the abbreviated VitalpacTM early warning score (ViEWS) after admission to hospital of 18,853 acutely ill medical patients. Resuscitation 2013;84:13-20.

14. Prytherch DR, Smith G, Schmidt PE, Featherstone P. ViEWS-Towards a national early warning score for detecting adult inpatient deterioration. Resuscitation 2010;81:932-7.

15. Bulut M, Cebicci H, Sigirli D, et al. The comparison of modified early warning score with rapid emergency medicine score: a prospective multicentre observational cohort study on medical and surgical patients presenting to emergency department. Emerg Med J 2013. [Epub ahead of print].

16. Churpek M, Yuen TC, Edelson DP. Risk stratification of hospitalized patients on the wards. Chest 2013;143: 1758-65.

17. Agenzia Sanitaria e Sociale Regionale Emilia Romagna. Requisiti specifici per l'accreditamento delle strutture di medicina interna; Oct 2011. Available from: http://assr.regione.emilia-romagna.it/it/servizi/pubblicazioni/legislazione-e-atti-amministrativi/requisitiaccreditamento/requisiti-specifici

18. Fullerton J, Price CL, Silvey NE, et al. Is the modified early warning score (MEWS) superior to clinician judgement in detecting critical illness in the pre-hospital environment? Resuscitation 2012;83:557-62.

19. Royal College of Physician. National Early Warning Score (NEWS) - Standardising the assessment of acuteillness severity in the NHS. Report of a working party; July 2012. Available from: https://www.rcplondon. ac.uk/resources/national-early-warning-score-news

20. Chesi G, Boni F. Ospedali e modelli organizzativi per intensità di cure: il punto di vista dell'internista. Ital J Med 2012;6:63-71.

21. Nardi R, Arienti V, Nozzoli C, Mazzone A. Organizzazione dell'ospedale per intensità di cure: gli errori da evitare. Ital J Med 2012;6:1-13.

22. Nardi R, Mathieu G, Berti F, et al. Evaluation models and items of clinical competence for the hospital physicians in internal medicine. Ital J Med 2011;55:S3-13. 


\title{
Nella Medicina Interna ospedaliera vogliamo un sistema sanitario pubblico sostenibile: considerazioni e proposte di FADOI per ridurre le spese inappropriate
}

\author{
Roberto Nardi, ${ }^{1}$ Daniele Borioni, ${ }^{1}$ Angelo Pasquale, ${ }^{1}$ Giuseppe Chesi, ${ }^{2}$ Franco Berti, ${ }^{3}$ Leonardo M. Fabbri, ${ }^{4}$ \\ Ido Iori, ${ }^{5}$ Giovanni Mathieu, ${ }^{6}$ Antonino Mazzone, ${ }^{7}$ Mauro Campanini, ${ }^{8}$ Carlo Nozzoli, ${ }^{9}$ Andrea Fontanella, ${ }^{10}$ \\ a nome del Gruppo FADOI sulle decisioni appropriate nei Reparti di Medicina Interna
}

\begin{abstract}
${ }^{1}$ Azienda USL di Bologna, Ospedale Maggiore, Medicina Interna, Bologna; ${ }^{2}$ Dipartimento Internistico Area Sud Montana, Ospedale Magati di Scandiano (RE); ${ }^{3}$ Medicina Interna 3, AO San Camillo-Forlanini, Roma; ${ }^{4}$ Dipartimento di Oncoematologia e Malattie Respiratorie, Università di Modena e Reggio Emilia; ${ }^{5}$ Medicina Interna e Centro Emostasi e Trombosi, AO di Reggio Emilia, Arcispedale Santa Maria Nuova, Reggio Emilia; ${ }^{6}$ Medicina Interna, Ospedale E. Agnelli, Pinerolo (TO); ${ }^{7}$ Dipartimento di Area Medica, UOC Medicina Interna, Ospedale Civile, Legnano (MI); ${ }^{8}$ Medicina Interna 2 - Dipartimento Medico, AO Maggiore della Carità, Novara; ${ }^{9}$ Medicina Interna e d'Urgenza, AOU Careggi, Firenze; ${ }^{10}$ Dipartimento di Medicina, Ospedale Madonna del Buon Consiglio Fatebenefratelli, Napoli, Italia
\end{abstract}

\section{RIASSUNTO}

Tutti i paesi si trovano ad affrontare il problema di come sostenere la qualità delle cure, a fronte della riduzione dei bilanci sanitari ed in presenza di un costante aumento della speranza di vita e con una significativa domanda crescente di assistenza sanitaria da parte della popolazione più anziana cronicamente malata. L'attuale orientamento delle più recenti misure legislative si impronta in gran parte ad una politica di riduzione dei costi. Con questo approccio, introdotto in maniera indiscriminata, a prescindere dai risultati ottenuti da ciascuna organizzazione sanitaria regionale secondo criteri di valutazione espliciti, si mettono a rischio i servizi, con la possibilità di una drastica riduzione dei posti letto in ospedale, con evidenti svantaggi a discapito dei gruppi di popolazione più vulnerabili. Nella nostra Società Scientifica di Medicina Interna (Federazione delle Associazioni dei Dirigenti Ospedalieri Internisti, FADOI), vogliamo sostenere che una medicina essenziale è ancora un obiettivo da realizzare. Siamo alla ricerca di modi originali per attuare una Medicina Interna basata su una metodologia clinica saggia ed efficace, da applicare nella cura dei pazienti ricoverati in base alle loro reali esigenze. Crediamo fortemente che un'agenda comune fra la Medicina Interna e la salute pubblica, attraverso una visione condivisa tra cittadini, professionisti e decisori nella pianificazione delle cure, possa essere una grande opportunità per qualsiasi riforma sanitaria. Il futuro del sistema sanitario non può limitarsi alla riduzione dei costi, ma dovrebbe mirare a fornire la migliore assistenza sanitaria possibile in relazione al denaro speso. Anche in questo periodo di austerità, possono ancora essere trovate nuove opportunità e i medici devono continuare a sforzarsi per affrontare questa sfida.

Corrispondente: Roberto Nardi, via C. Pavese 16/2, 40141 Bologna, Italia.

Tel.: +39.335.8291342. E-mail: nardidoc48@gmail.com

Parole chiave: medicina sostenibile, spending review, medicina interna, spese inappropriate.

Ringraziamenti: si ringrazia la dott.ssa S. Nardi per la preziosa collaborazione fornita nella revisione italiana dell'articolo originale.

Modificato da: R. Nardi, F. Berti, L.M. Fabbri, et al. Toward a sustainable and wise healthcare approach: potential contributions from hospital Internal Medicine Departments to reducing inappropriate medical spending. Ital J Med 2013;7:65-81.

Articolo pubblicato secondo la Creative Commons Attribution NonCommercial 3.0 License (CC BY-NC 3.0).

CCopyright R. Nardi et al., 2014

Licensee PAGEPress, Italy

QUADERNI - Italian Journal of Medicine 2014; 2:29-48

\section{Introduzione}

Secondo gli ultimi dati del Ministero della Salute, in Italia, negli ultimi dieci anni la spesa sanitaria è aumentata da 76 a poco meno di 113 miliardi di euro, con una crescita di circa 37 miliardi di euro. I dati del governo per l'anno 2011 hanno dimostrato che la spesa sanitaria pubblica italiana è equivalente al 7,1\% del prodotto interno lordo (PIL) e a $€ 1842$ pro capite all'anno. ${ }^{1}$ In un confronto tra Italia e l'Unione Europea, l'incidenza della spesa sanitaria pubblica sul PIL nel 2009 è stata del 7,3\%, rispetto all' $8,2 \%$ registrato in Europa, evidenziando così il fatto che, in media, in Italia, spendiamo meno per la sanità rispetto ad altri paesi europei. Tutti i paesi si trovano ad affrontare il problema di come sostenere la qualità delle cure, malgrado la riduzione dei bilanci sanitari, in presenza di un persistente aumento della speranza di vita, e con una significativa domanda crescente di assistenza sanitaria da parte della popolazione più anziana, costituita prevalentemente da pazienti cronicamente 
malati. ${ }^{2} \mathrm{Da}$ oltre un decennio, in Italia e nell'Unione Europea il sistema sanitario ha subito riforme che mirano a razionalizzare le risorse e a creare restrizioni sulle spese. Le misure adottate, in gran parte sottoforma di tagli, vengono legittimate dalla giusta causa del contenimento di costi. Con questo approccio, introdotto in maniera indiscriminata, a prescindere dai risultati ottenuti da ciascuna organizzazione sanitaria regionale secondo criteri di valutazione espliciti, si mettono a rischio i servizi, non si garantisce il turnover del personale medico ed infermieristico, si rischia una drastica riduzione del numero dei posti letto in ospedale, con evidenti svantaggi, particolarmente a discapito dei gruppi di popolazione più vulnerabili. Queste riduzioni quasi automatiche nelle modalità di contenimento della spesa sanitaria sono state da alcuni definite come veri e propri sequestri, ${ }^{3}$ con riduzione dei fondi destinati agli ospedali, alle case di cura e all'acquisizione di personale. ${ }^{4}$ In realtà, il rapporto tra sanità, costi e qualità non è stato ancora pienamente compreso. Sono necessari ulteriori studi per capire quali tipi di spesa siano più efficaci nel migliorare la qualità e quali, invece, rappresentino sprechi. ${ }^{5}$

\section{La medicina difensiva}

Nella pratica medica corrente un problema preoccupante è l'uso della medicina difensiva, definita come l'insieme di tutte le attività mediche che non comportano benefici diretti agli assistiti, ma che vengono comunque fornite allo scopo di ridurre al minimo per i medici i rischi di querele e richieste di danni da parte dei pazienti e dei loro famigliari. Ciò si verifica particolarmente in situazioni cliniche potenzialmente pericolose, che possono avere conseguenze legali e strascichi giudiziari, con una conseguente prescrizione precauzionale di inutili, a volte ridondanti, interventi diagnostico-terapeutici. ${ }^{6}$ La medicina difensiva è ampiamente praticata, con un impatto elevato sui costi dell'assistenza sanitaria. In un'indagine effettuata fra medici di 6 specialità ad alto rischio di contenzioso (medicina d'urgenza, chirurgia generale, ortopedia, neurochirurgia, ostetricia/ginecologia e radiologia), 9 su 10 degli intervistati hanno riferito di utilizzare pratiche difensive, con il rilievo che oltre il $90 \%$ di tutti gli intervistati ordina test clinici non necessari. ${ }^{7}$ In un sondaggio Gallup, i medici hanno attribuito il $34 \%$ dei costi sanitari complessivi alla medicina difensiva, evidenziando che il $21 \%$ della loro pratica professionale è di natura difensiva. In particolare, il 35\% degli esami diagnostici, il $29 \%$ dei test di laboratorio, il 19\% delle ospedalizzazioni, il 14\% delle prescrizioni e l' $8 \%$ degli interventi chirurgici sono effettuati per evitare cause legali. Questa indagine ha stimato che le pratiche di medicina difensiva costano agli Stati Uniti 650-850 miliardi di dollari ogni anno. ${ }^{8}$ Diverse strategie sono state proposte per contenere la spesa sanitaria nazionale dovuta alla medicina difensiva: una di queste è costituta dalla cosiddetta safe harbour strategy (strategia di un porto sicuro), che esime i medici da responsabilità in caso di adesione nella pratica clinica ad evidenze cliniche e linee guida. ${ }^{9}$ Purtroppo, una strategia simile può non essere sempre applicabile nei pazienti complessi ricoverati in medicina interna, dove l'incertezza è spesso all'ordine del giorno. Molti medici ritengono che la preoccupazione di un esito in malpractice dei loro atti comporti l'effettuazione di esami e procedure inutili. Essi credono altresì che il sistema sanitario dovrebbe tutelare i professionisti dai rischi derivanti dalla responsabilità medica, ogniqualvolta esistano proposte volte a promuovere azioni costo-efficaci, come ad esempio la promulgazione di linee guida: tali tutele dovrebbero però essere garantite. ${ }^{10}$ In ogni caso è necessario intervenire sui costi legati alla medicina difensiva, ${ }^{11}$ incidenti per una quota calcolata superiore al $10 \%$ sulla spesa sanitaria. Qualsiasi ragionevole proposta di riforma, magari con un patto tra Sistema sanitario Nazionale e Cittadini, potrebbe modificare l'attuale situazione, scoraggiare le pretese infondate e legittimare solamente quelle appropriate.

\section{L'appropriatezza in medicina clinica}

La maggior parte dei medici desiderano fornire cure appropriate ed esercitare la professione secondo comportamenti etici, ma una grande parte delle prestazioni sanitarie fornite in tutto il mondo è inappropriata. L'appropriatezza è tutto ciò che è adatto, conveniente e adeguato. Questa parola è stata menzionata in varie misure legislative in Italia, fin dagli anni 2000. ${ }^{12}$ Essa differisce da altre caratteristiche utilizzate per la valutazione della qualità dei servizi sanitari, quali performance, correttezza, efficienza, efficacia (Tabella 1 e Figura 1). Nello stesso concetto di appropriatezza esistono diverse interazioni (Figura 1).

\section{Futilità medica: una definizione non univoca}

Anche se i medici riconoscono frequentemente che interventi da loro proposti sono inutili in molti casi, continuano tuttavia a prescriverli, sostenendo una serie di ragioni, finalizzate a: i) mantenere la disponibilità del paziente; ii) proteggersi dalla eventuale critica di negligenza; iii) evitare la negazione di un trattamento in quanto ritenuta inopportuna e immorale; iv) garantire la priorità della cura del paziente. ${ }^{16}$

La maggior parte dei medici deve affrontare decisioni difficili, tra cui le scelte terapeutiche problematiche nei casi di pazienti con prognosi infausta nel breve termine. Il concetto di futilità in Medicina è empirico, senza esplicite soglie definibili a priori per la sua determinazione. ${ }^{17}$ Secondo Scheinderman et al., un trattamento dovrebbe essere considerato futile 
quando i medici concordano che tale opzione è risultata inutile negli ultimi 100 casi (attraverso l'esperienza personale) o in base ai dati della letteratura. ${ }^{18}$ Non tutto ciò che è tecnicamente possibile è opportuno, così come non tutto ciò che potrebbe essere fatto dovrebbe essere attuato. ${ }^{19}$ Il dubbio se trattare o non trattare dovrebbe essere affrontato non solamente in base al giudizio di utilità di uno specifico esame e/o trattamento, ma su una valutazione complessiva dei pro e contro nell'intraprendere determinate cure e sull'idoneità del paziente di sottoporsi a trattamento, in base ad un giudizio clinico prognostico esplicito. ${ }^{20}$

\section{L'eccesso di diagnosi e la mercificazione della malattia}

L'eccesso di diagnosi (overdiagnosis) si realizza quando, in assenza di sintomi, viene diagnosticata una malattia che in seguito non causerà né alcuna sintomatologia né morte precoce. ${ }^{21,22}$ Essa deriva da un'esagerata medicalizzazione, con conseguente trattamento eccessivo e ridondante, ma anche dall'accapponarsi in una diagnosi obbligata (diagnosis creep), per cui se viene identificata un'ipotesi di malattia i medici cominciano a vederla ovunque. In altri casi si spostano le soglie di valutazione della malattia e/o del rischio (ad esempio, per l'ipertensione arteriosa o per il colesterolo), fino a considerare ammalate persone sane o con problemi lievi o a basso rischio di malattia. ${ }^{23}$ L'eccesso di diagnosi inevitabilmente comporta l'esposizione ai danni potenziali del trattamento, a fronte di nessun beneficio. ${ }^{24}$ Addirittura si giunge alla riclassificazione delle malattie, fino a casi di vera e propria mercificazione della salute. L'ampliamento dei confini delle malattie potenzialmente curabili con farmaci, al fine di allargare i mercati a

\begin{tabular}{|c|c|c|c|}
\hline Correttezza & $\begin{array}{c}\text { Se } \mathrm{i} \\
\text { comportamenti }\end{array}$ & a priori & $\begin{array}{l}\text { Rispettano le } \\
\text { regole }\end{array}$ \\
\hline Efficacia & Se l' azione & 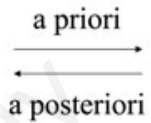 & $\begin{array}{l}\text { Raggiunge lo } \\
\text { scopo }\end{array}$ \\
\hline $\begin{array}{l}\text { Efficienza } \\
\text { tecnica }\end{array}$ & Se lo strumento & 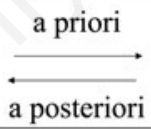 & $\begin{array}{l}\text { È quello più } \\
\text { adatto }\end{array}$ \\
\hline $\begin{array}{l}\text { Efficienza } \\
\text { allocativa }\end{array}$ & Se le risorse & a posteriori & $\begin{array}{c}\text { Hanno un impiego } \\
\text { ottimale rispetto alle } \\
\text { alternative }\end{array}$ \\
\hline
\end{tabular}

Figura 1. Interazioni dell'appropriatezza. Fonte: Cislaghi, 2005. ${ }^{15}$

Tabella 1. Appropriatezza, prestazioni, efficienza, efficacia.

\begin{tabular}{|c|c|c|c|}
\hline \multicolumn{2}{|l|}{ Appropriatezza } & \multicolumn{2}{|c|}{$\begin{array}{l}\text { Fare la cosa giusta, al paziente giusto, al momento giusto, nel contesto adeguato, dal } \\
\text { professionista giusto, dal punto di vista terapeutico diagnostico e del comportamento clinico }\end{array}$} \\
\hline & & In relazione agli obiettivi & In relazione all'esecuzione \\
\hline & & Fare cose giuste nel modo corretto & Fare cose giuste nel modo sbagliato \\
\hline & & Fare cose sbagliate nel modo corretto & Fare cose sbagliate nel modo sbagliato \\
\hline Performance & & $\begin{array}{l}\text { Il modo giusto di fare qualcosa, con riferiment } \\
\text { richieste nel ruolo professionale }\end{array}$ & alla competenza clinica ed alle competenze \\
\hline \multirow[t]{4}{*}{ Efficienza } & Efficienza tecnica & $\begin{array}{l}\text { Gli output non possono essere prodotti } \\
\text { con meno di alcuni input }\end{array}$ & Opportunità di ridurre gli sprechi \\
\hline & $\begin{array}{l}\text { Allocazione efficiente } \\
\text { di risorse }\end{array}$ & $\begin{array}{l}\text { Le risorse vengono impiegate in maniera } \\
\text { ottimale rispetto a qualsiasi altra alternativa } \\
\text { disponibile }\end{array}$ & Opportunità di ridurre gli sprechi \\
\hline & Produzione efficiente & $\begin{array}{l}\text { Gli output (i risultati) non possono essere } \\
\text { prodotti ad un costo più basso }\end{array}$ & Opportunità di risparmiare denaro \\
\hline & Efficienza sociale & $\begin{array}{l}\text { Nessuna persona può migliorare la propria } \\
\text { condizione senza che qualcun altro peggiori } \\
\text { la sua (efficienza di Pareto-ottimo paretiano) }{ }^{13}\end{array}$ & $\begin{array}{l}\text { Opportunità per massimizzare i valori } \\
\text { etico sociali }\end{array}$ \\
\hline \multicolumn{2}{|l|}{ Efficacia } & \multicolumn{2}{|c|}{$\begin{array}{l}\text { La misura per cui un intervento specifico, una procedura o un servizio produce l'effetto } \\
\text { desiderato, in condizioni ideali (ambiente controllato, laboratorio/circostanze sperimentali) }\end{array}$} \\
\hline \multicolumn{2}{|l|}{ Effectiveness } & \multicolumn{2}{|c|}{$\begin{array}{l}\text { La misura per cui i risultati pianificati e gli obiettivi sono raggiunti a seguito di una attività, } \\
\text { una strategia, un intervento o un'iniziativa intesa ad ottenere l'effetto desiderato, in } \\
\text { circostanze normali non controllate }\end{array}$} \\
\hline \multirow{2}{*}{\multicolumn{2}{|c|}{ CE dell'intervento sanitario }} & \multicolumn{2}{|l|}{ Benefici associati ad un intervento e relativi costi } \\
\hline & & \multicolumn{2}{|c|}{ Rapporto medio $\mathrm{CE}=$ costo intervento - costi evitati da interventi/benefici intervento } \\
\hline
\end{tabular}


scopo di profitto ${ }^{25}$ costituisce un'evidente ulteriore causa di spreco per la salute pubblica. In questo ambito sono state incluse la disfunzione erettile, la disfunzione sessuale femminile, la menopausa, la sindrome premestruale, i disturbi bipolari, i deficit di attenzione, l'iperattività, la sindrome delle gambe senza riposo, l'osteoporosi, la timidezza sociale (chiamata anche disturbo d'ansia e fobia sociale), la sindrome dell'intestino irritabile, la calvizie. ${ }^{26}$

\section{L'(ab)uso dei servizi di emergenza ospedalieri e i (re)ricoveri ospedalieri non programmati}

I Dipartimenti di Medicina Interna in Italia sono gravati della maggior parte dei ricoveri per pazienti difficili e complessi. ${ }^{27}$ Circa il $40 \%$ dei pazienti autonomi dal punto di vista funzionale che accedono al Pronto Soccorso potrebbero essere gestiti dal medico di Medicina generale. ${ }^{28}$ Secondo il rapporto del Ministero della Salute, la popolazione anziana in Italia rappresenta circa il $40 \%$ dei ricoveri ospedalieri, cui corrispondono la metà delle di giornate di degenza con i relativi costi. ${ }^{29}$ L'interazione tra invecchiamento, malattie croniche e loro riacutizzazioni o malattie acute intercorrenti, insieme alla vulnerabilità socio-economica, definisce un categoria di pazienti, per lo più anziani, che sono frequenti utilizzatori (frequent users) dell'ospedale, condivisi da diversi specialisti, spesso non in grado di fornire risposte adeguate alla gestione del paziente, a volte ricoverati in ospedale in attesa di soluzione ai problemi clinicosociali (Tabella 2).

In uno studio condotto tra il 1990 e il 2004 è stato documentato un aumento del $54 \%$ del numero totale di accessi in ospedale, con incremento sproporzionato del $198 \%$ di pazienti di età superiore ai 70 anni e del $671 \%$ in quelli di età superiore a $90 ! .{ }^{31}$ I ricoveri ospedalieri non programmati e la gestione post-dimissione costituiscono importanti problemi per i reparti di Medicina interna. Da un'analisi riferita ai dati dei costi

Tabella 2. Ricoveri ospedalieri e gestione dei pazienti complessi: alcune definizioni.

Revolving door syndrome: il ripetersi a cadenze più o meno brevi di passaggi del malato, in genere anziano, fragile, pluripatologico, tra l'Ospedale ed il domicilio e viceversa

Frequent user: il paziente che accede con elevata frequenza all'utilizzo dei servizi sanitari ospedalieri

Sindrome del ping-pong: passaggio del paziente da uno specialista all'altro, mancata presa in carico

Malati ecumenici: pazienti visti da numerosi specialisti, nessuno dei quali, comunque, si assume l'onere della gestione del paziente

Bed-blockers: pazienti ricoverati per problemi acuti che restano ricoverati perché in attesa di una diversa collocazione

Fonte: Cioni, 2011. ${ }^{30}$ relativi a ricoveri ripetuti presso l'Ospedale Maggiore di Bologna nel 1997 risultava che il 61\% degli importi calcolati sulla degenza ospedaliera per anno era ascrivibile a re-ricoveri (Figura 2).

Nello studio TEMISTOCLE sullo scompenso cardiaco era stata documentata una percentuale di re-ricoveri non programmati a 6 mesi pari al $45,4 \%$ nei reparti di Medicina Interna. ${ }^{32}$ Nello studio CONFINE, relativo a pazienti ammessi in un reparto di Medicina Interna per scompenso cardiaco, su 1444 casi considerati solamente il $35,1 \%$ riguardava pazienti al primo ricovero; i restanti casi $(64,9 \%)$ erano ascrivibili a ricoveri successivi al primo. ${ }^{33,34}$ Da uno studio spagnolo effettuato in 999.089 pazienti ricoverati in reparti di Medicina Interna è stata rilevata una prevalenza di riammissioni non programmate entro un mese dalla dimissione pari al $12,4 \% .{ }^{35}$ In un recente studio italiano effettuato in quasi 12.000 pazienti in un reparto di Medicina Interna è stato documentato un tasso di re-ricovero entro 30 giorni dalla dimissione del $18 \%$ e del $15 \%$ rispettivamente nei casi di scompenso cardiaco e polmonite. ${ }^{36}$ Nell'analisi delle cause di ricoveri ripetuti un possibile modello è quello rappresentato nella Figura $3 . .^{37}$

Nonostante il fatto che una quota ragionevole di ricoveri e re-ricoveri può essere evitata, l'evidenza suggerisce che i principali fattori che determinano alti tassi di re-ricovero entro 30 giorni sono la composizione della popolazione, costituita da pazienti anziani complessi e le risorse del territorio nella comunità locale. Nessuno di questi fattori è correlabile all'organizzazione o ai comportamenti professionali del personale ospedaliero, né tantomeno può essere modificato dall'ospedale. ${ }^{38}$

\section{Le procedure non necessarie}

Abbiamo trascorso gli ultimi 20 anni introducendo le migliori linee guida cliniche e i percorsi diagnostico-terapeutici sulla base delle evidenze disponibili, ma molto lavoro è ancora necessario per colmare il di-

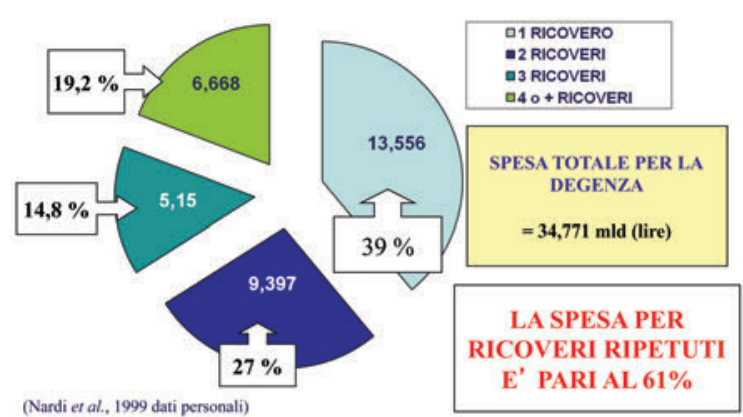

Figura 2. Ricoveri singoli e ripetuti in Medicina Interna importi calcolati sulla degenza (in mld di lire) (Ospedale Maggiore di Bologna, anno 1997). 
vario tra ciò che dobbiamo (o dovremmo) fare e ciò che effettivamente facciamo. ${ }^{39}$ Assumere una decisione autonoma quando mancano le evidenze e/o in condizioni di incertezza non significa che si deve decidere in base alle abitudini. Un esempio paradigmatico di comportamenti inappropriati (nonché inopportuni) è dato dalla richiesta di procedure diagnostiche non necessarie.

\section{La prescrizione degli esami diagnostici}

Nella diagnosi delle malattie il contributo della medicina basata sull'evidenza è, nella maggior parte dei casi, molto limitato. È necessario un migliore utilizzo degli accertamenti richiesti, sia quando essi siano over-ordered che under-ordered.$^{40}$ Da questo punto di vista, l'introduzione di una valutazione delle tecnologie sanitarie è molto utile. Questa viene intesa come attività multidisciplinare che sistematicamente esamina la sicurezza, l'efficacia clinica e l'efficienza, i costi ed il rapporto costo-efficacia, le implicazioni organizzative, le conseguenze sociali, le considerazioni legali ed etiche riguardo all'applicazione di una tecnologia sanitaria, che può essere un farmaco, un dispositivo medico o una procedura clinica e/o chirurgica. L'attenzione deve essere rivolta all'allocazione delle strumentazioni di alto costo rispetto ai potenziali bacini di utenza e alla valutazione non solamente dell'efficacia clinica (rispetto ad alternative disponibili di trattamento) ma anche a quella del costo-efficacia (questi miglioramenti negli esiti sani- tari sono da ritenersi commisurati ai costi aggiuntivi della tecnologia proposta?). ${ }^{41}$

\section{Le indagini radiologiche}

In ambito radiologico le inappropriatezze sono evidenti. ${ }^{42}$ Secondo la Commissione Europea per le Linee Guida dell'imaging vi è un crescente utilizzo ma anche una crescente inappropriatezza, calcolata in almeno il $30 \%$ dei casi, della diagnostica per immagini, soprattutto di quella a più alto carico radiologico (scintigrafia, TC multistrato, TC-PET). ${ }^{43}$ Uno studio italiano effettuato sulle prestazioni radiologiche ambulatoriali ha mostrato che solamente il $56 \%$ degli esami effettuati era appropriato, con un fatturato totale degli esami di 257.317 euro ed un onere dovuto agli esami non appropriati pari a 94.012 euro $(36,5 \%) .{ }^{44}$ Parecchi fattori influenzano le richieste dei medici, tra questi, l'invecchiamento della popolazione, i progressi nella tecnologia per immagini e la loro aumentata disponibilità, le aspettative dei pazienti, l'incertezza dei professionisti, i vincoli di tempo e la medicina difensiva. Secondo i dati WONCA le cause che stanno alla base di richieste inappropriate di accertamenti radiologici sono la medicina difensiva $(39,56 \%$ dei casi), la mancanza di coordinamento tra medico di Medicina generale e specialisti $(26,20 \%)$, la non indicazione dell'esame rispetto al quesito diagnostico $(17,77 \%)$, l'accondiscendenza alle insistenze dei pazienti $(16,47 \%){ }^{45}$ Ripetere un esame a seguito di indagini già effettuate è un fenomeno comune. Fra i pazienti sotto-

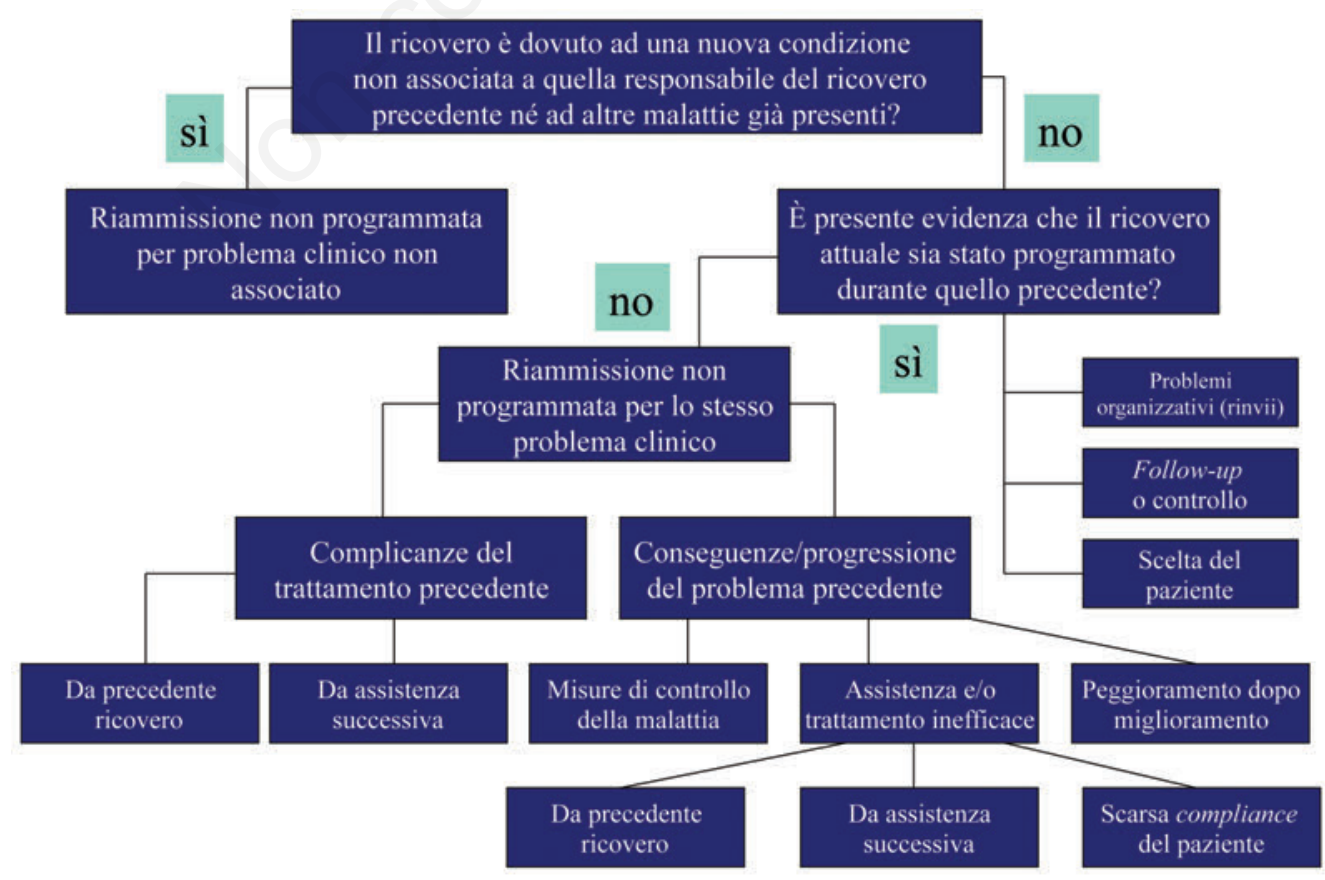

Figura 3. Modello di analisi dei ricoveri ripetuti in ospedale. Fonte: Ludke et al., $1993 .{ }^{37}$ 
posti a ecocardiografia, il 55\% ha avuto un secondo test entro tre anni; analogamente il $44 \%$ dopo esami di imaging, il $49 \%$ dopo test di funzionalità polmonare, il $46 \%$ per una TC del torace, il $41 \%$ dopo cistoscopia, e il 35\% dopo endoscopia del tratto digestivo superiore. ${ }^{46}$ Altri esempi di indagini con poco o nessun valore aggiunto sono lo screening per il cancro del collo dell'utero nelle donne a basso rischio di oltre 65 anni o in quelle isterectomizzate per malattia benigna. Ugualmente eseguire studi di imaging come il test diagnostico iniziale in pazienti con bassa probabilità di tromboembolia venosa, effettuare screening per malattia polmonare cronica ostruttiva con spirometria in individui senza sintomi respiratori ${ }^{47}$ sono esempi di indagini inappropriate. In un sondaggio nazionale rappresentativo il $42 \%$ di medici americani delle cure primarie ritiene che i pazienti stiano ricevendo troppe cure. I fattori più importanti addotti a giustificazione di tale pratica erano le preoccupazioni relative una possibile negligenza $(76 \%)$, il giudizio sulle prestazioni cliniche effettuate (52\%), e il tempo insufficiente dedicato ai pazienti (40\%). Buona parte degli esami diagnostici, pari a circa il $39 \%$, potrebbe essere ridotta se non venisse generata dalle indicazioni dei medici sub specialisti. ${ }^{48}$ Le decisioni sulle indagini diagnostiche da effettuare non possono essere assunte in base ad una lista di diversi accertamenti richiesti da molti specialisti che intervengono nella gestione condivisa dei pazienti complessi. I medici di Medicina generale e gli internisti ospedalieri devono selezionare le reali esigenze dei loro pazienti, assumendo la responsabilità del giudizio finale sull'adeguatezza delle procedure/indagini proposte (Tabella 3) e della regia del caso. ${ }^{49,50}$

In alcuni casi l'impiego di alcune procedure relativamente semplici può consentire di evitare il ricorso ad ulteriori indagini costose, come, ad esempio, l'ecografia del torace per valutare un quadro di wet lung in caso di dispnea acuta.

\section{Test di laboratorio}

Una delle aree di maggior crescita di richiesta di prestazioni in ambito sanitario è quella relativa alle in-

\section{Tabella 3. L'indice di in appropriatezza. ${ }^{46-47}$}

Prima di chiedere qualsiasi indagine, accertamento o esame, il clinico dovrebbe sempre chiedersi:

1. L'esame proposto aggiunge valore nel percorso di cura del paziente, tale da modificare la gestione clinica?

2. L'esame è già stato effettuato in precedenza?

3. Quali potenziali rischi corre il paziente nel sottoporsi all'indagine?

Se la risposta alla prima domanda non è esplicitamente affermativa ed emergono dubbi dalle altre domande, si pone con grande probabilità la inappropriatezza dell'esame che si vorrebbe effettuare dagini di laboratorio. ${ }^{51,52}$ L'abuso di risorse di laboratorio è ampiamente diffuso nella pratica ospedaliera, particolarmente nell'ambito dei dipartimenti di emergenza. Si calcola che più del $30 \%$ delle indagini, molte delle quali ripetute, potrebbero essere evitate. Le ragioni dell'eccessiva ed inadeguata richiesta di esami includono ancora una volta, un'attitudine difensiva, ma anche paura o incertezza decisionale, mancanza di esperienza, l'impiego sbagliato di protocolli e linee guida, abitudini personali o locali, un background culturale inadeguato, oltre che la mancanza di consapevolezza da parte del medico del costo degli esami. ${ }^{53}$ Altri fattori che contribuiscono all'inadeguata richiesta di esami laboratoristici sono l'età del paziente (più di 65 anni), la durata di degenza superiore a sette giorni, la difficoltà del caso o la incapacità di definire una diagnosi certa. ${ }^{54,55}$ Le richieste di indagini di laboratorio inappropriate possono essere controllate automaticamente sulla base di algoritmi (reflex testing) o direttamente da parte dei medici di laboratorio nell'interazione con i clinici. ${ }^{56}$ In alcuni casi, è meglio investire per disinvestire. Introdurre l'impiego di alcuni test può consentire di evitare il ricorso ad ulteriori indagini costose, come, ad esempio, il peptide natriuretico per l'esclusione di insufficienza cardiaca o la calprotectina fecale per l'esclusione di una malattia infiammatoria intestinale, con un minor ricorso rispettivamente alle più costose ecocardiografie e colonscopie, ovvero il dosaggio della procalcitonina nella diagnosi di infezione batterica clinicamente rilevante e nelle sepsi ${ }^{57}$ o del l'1-3)- $\beta$-D-glucano nella diagnosi precoce delle infezioni fungine invasive. ${ }^{58}$

\section{La qualità delle cure}

I possibili rischi di un confronto econometrico fra diverse organizzazioni/reparti se questo viene effettuato in base a bias di selezione

Qualsiasi analisi comparativa di tipo econometrico tra i diversi reparti dovrebbe descrivere i pazienti coinvolti. Nei nostri reparti di Medicina Interna la tipologia di pazienti ricoverati impone un confronto con altri setting assistenziali ospedalieri sulla base delle caratteristiche di complessità degli ammalati: numerosità del campione, età, sesso, compresenza di malattie croniche, politerapia, fragilità, instabilità clinica, dipendenza funzionale, disfunzione cognitiva, stato nutrizionale e alimentazione, tono affettivo, contesto socio-economico e famigliare, eccetera. Prima di giudicare qualsiasi esito di cura positivo o negativo in reparti sub-specialistici rispetto ai reparti di Medicina Interna, indipendentemente dalla specialità medica considerata, dobbiamo descrivere gli strumenti presi in considerazione per definire la complessità dei pazienti e per cercare di omogeneizzare le popolazioni in confronto, descrivendo le risorse disponibili per la loro gestione. È necessario evitare qualsiasi faziosità 
quando si confrontano diverse popolazioni; non si dovrebbe confondere i risultati fra diversi reparti se quelli ad indirizzo sub-specialistico, per lo più orientati verso patologie d'organo o di apparato, trattano malati meno complessi di quelli ricoverati nei reparti di medicina interna. Qualsiasi analisi ospedaliera basata sulla valutazione dei diagnosis-related groups, durata di degenza, tasso di re-ricovero a 30 giorni e mortalità dovrebbe affiancarsi alla descrizione dei soggetti analizzati, insieme a diversi altri indicatori di esito, quali la soddisfazione percepita da parte dei pazienti e delle loro famiglie, il miglioramento della qualità di vita, le modalità di gestione del dolore e delle cure palliative, il rispetto delle aspettative e delle volontà del paziente in riferimento alle cure proposte $\mathrm{e}$ la possibilità di presa in carico post dimissione.

\section{La qualità d'impiego dei farmaci: prescrizioni inappropriate, ma non solo}

In letteratura sono descritti molti criteri di valutazione di una prescrizione inappropriata dei farmaci, in riferimento ai diversi concetti di errata, esagerata e ridondante o insufficiente/mancata prescrizione (rispettivamente: misprescribing, overprescribing e underprescribing) (Tabella 4) . Nel rapporto OsMed gennaio - settembre 2013 per la prima volta viene introdotto il monitoraggio degli indicatori di appropriatezza d'uso dei medicinali, cui si rimanda. ${ }^{59}$

Nel Regno Unito una prescrizione su tre viene effettuata per pazienti ultra-65enni, e nel $90 \%$ di questi casi si tratta di ricette ripetute per terapie croniche. Gli anziani sono particolarmente suscettibili agli effetti collaterali dei farmaci in relazione ai cambiamenti farmacocinetici e farmaco-dinamici.

Un riferimento fondamentale per le prescrizioni potenzialmente inappropriate nell'anziano sono i criteri di Beers, recentemente aggiornati dalla American Geriatric Society. ${ }^{61}$ La politerapia, determinata dalla presenza di molteplici problemi coesistenti nello stesso individuo, può causare una scarsa adesione alla terapia, oltre che possibili effetti collaterali e interazioni farmacologiche. Dobbiamo considerare non solo l'appropriatezza delle nostre prescrizioni, ma anche altri elementi che possono influenzare la spesa sanitaria. Troppi prescrittori sono coinvolti nella gestione di un singolo paziente, vi è un evidente sottoutilizzo di farmaci equivalenti rispetto alle altre regioni d'Europa, non vi è certezza di aderenza alle terapie, sono possibili interruzioni di trattamento ignote al medico, molti farmaci prescritti sono potenzialmente inadeguati per i pazienti più anziani, la conservazione dei farmaci può essere inadatta, molti farmaci prescritti sono acquistati ma non assunti dai pazienti. Tutti questi fattori possono influenzare, direttamente o indirettamente i costi sanitari ${ }^{62}$ (Tabella 5).

\section{Prima l'efficacia poi l'efficienza}

Molte inefficienze derivano dalle modalità con cui viene gestita l'assistenza sanitaria, secondo un principio fondante che privilegia il primato dell'efficacia per il raggiungimento dell'efficienza. Per un frugale, ma efficace, sistema sanitario sono state proposte sei regole essenziali $^{78}$ (Tabella 6).

\section{Dobbiamo solamente tagliare e disinvestire?}

L'onere crescente delle tecnologie sanitarie, con le conseguenti spese, è un argomento di discussione nella pianificazione dei servizi per la salute in tutto il mondo. Le proposte emergenti si basano sulla necessità di un miglior coordinamento delle cure, sull'efficienza amministrativa e l'eliminazione degli interventi inutili, ${ }^{79}$ ma nella maggioranza dei casi predomina un orientamento alla revisione della spesa, con disinvestimenti nella sanità che potrebbero avere effetti devastanti, soprattutto nei pazienti più poveri $\mathrm{e}$ vulnerabili. ${ }^{80}$ Questo approccio rischia di associarsi ad una politica di mera riduzione dei costi piuttosto che ad una strategia coordinata volta ai massimizzare i risultati degli investimenti. ${ }^{81}$ È necessaria una visione in positivo nella pianificazione dell'impiego delle limitate risorse disponibili. Alcuni possibili modi per attuare (dis)investimenti costruttivi sono rappresentati nella Tabella 7.82

Tabella 4. Misprescribing, overprescribing and underprescribing.

\begin{tabular}{ll}
\hline Misprescribing & $\begin{array}{l}\text { Prescrizione di un farmaco che aumenta significativamente il rischio di una reazione avversa. Esso comprende: } \\
\text { - La prescrizione di dosi, intervalli, modalità e via di somministrazione o durata del trattamento errati } \\
\text { - L'uso di farmaci che possono causare effetti clinicamente significativi indesiderati o interazioni tra diversi farmaci } \\
\text { o tra il farmaco impiegato e lo stato patologico del paziente, per cui dovrebbero essere considerate alternative più si- } \\
\text { cure, ma ugualmente efficaci }\end{array}$ \\
\hline Overprescribing & Prescrizione di farmaci per i quali non esiste alcuna chiara indicazione clinica \\
\hline Underprescribing & $\begin{array}{l}\text { Sottoutilizzo o omissione di farmaci potenzialmente benefici che sono clinicamente indicati per il trattamento o la } \\
\text { prevenzione di una malattia }\end{array}$
\end{tabular}

Fonte: Simon, 2008. ${ }^{60}$ 
Tabella 5. Alcuni fattori che condizionano i costi correlati alle prescrizioni di farmaci.

Adesione a cambiamenti nello stile di vita

Compliance $^{65}$ e non aderenza alle prescrizioni di farmaci*

Convincere i nostri pazienti a cambiare i comportamenti nello stile di vita è il modo migliore per ottenere maggiori benefici in termini di salute con una spesa sociale minima, come, ad esempio, nella riduzione dell'incidenza del diabete 63,64

Regimi farmacologici complessi possono essere fonte di confusione e determinare una scarsa aderenza alla terapia prescritta

Per questo fino al 50\% dei pazienti anziani può non assumere la terapia prevista $^{66,67}$

La non aderenza alle cure, con i costi conseguenti, costituisce un importante indicatore di qualità, significativamente associato a risultati negativi, quali ricoveri ospedalieri indesiderati

Prescrizioni diverse dal solito o confezioni diverse da quelle abitualmente assunte dal paziente possono contribuire ad una scarsa aderenza ed errori, con possibili problemi di sicurezza e di efficacia

Ulteriori elementi importanti per la popolazione anziana sono dati dall'opportunità di dover ridurre le dosi di alcuni farmaci, per le modificate condizioni farmacocinetiche/farmacodinamiche, nonché di tener conto della possibile presenza di compromissione visiva e motoria (ad esempio, nella prescrizione di soluzioni in gocce) e di interazioni fra farmaci per la poli-terapia ${ }^{68}$

Sottoutilizzo dei farmaci equivalenti

I farmaci generici sono ancora sottoutilizzati in Italia, con una conseguente mancata opportunità di ridurre ulteriormente i costi dell'assistenza sanitaria ${ }^{69}$

È stato calcolato che se nel 2007 tutti i potenziali utenti avessero utilizzato farmaci equivalenti piuttosto che quelli branded, ne sarebbero conseguiti risparmi di circa 115 dollari (95\% IC: 127-124 dollari) per persona all'anno, con un risparmio totale della società di 5,78 miliardi di dollari ${ }^{70}$

Compliance e aderenza ai farmaci equivalenti I farmaci generici sono equivalenti ai corrispondenti farmaci di marca come principi attivi

Essi possono differire in alcune caratteristiche, quali colore o la forma della pillola, eccipienti, processi di fabbricazione. Le regole per definire la bioequivalenza tra farmaci non considerano questi aspetti ${ }^{71,72}$

Questi fattori possono però influenzare negativamente l'aderenza alla terapia, soprattutto nei paziente anziani che già assumono molti farmaci

Sospensione intempestiva e impropria del trattamento

L'aggiunta di nuovi farmaci non strettamente necessari può comportare il rischio di eliminazione da parte di un paziente non informato di altri medicinali essenziali (ad esempio diuretici o anticoagulanti), con possibili effetti negativi

Reazioni avverse ai farmaci (ADRs)

Le ADR causano un ricovero ospedaliero per circa il 6,5\% delle ammissioni in ospedale, con una durata di degenza media 8 giorni ed un costo annuo calcolato in 706 milioni di euro ${ }^{73,74}$

Il fattore più importante di rischio di ricovero in ospedale dovuto ad ADR è dato dal numero di farmaci che sono stati assunti ${ }^{75}$

Se vengono fatte molteplici prescrizioni da parte dei diversi medici che seguono in diverse occasioni il paziente è possibile che vengano prescritti farmaci tra loro incompatibili, se non ripetuti. L'introduzione di nuovi farmaci da parte di altri sub-specialisti, senza la supervisione del medico di medicina generale o senza esercitare la riconciliazione terapeutica può portare a pericolose sovrapposizioni

Lo spreco che deriva da questa incongruenza è stato stimato pari al 6-10\% sul totale costi di prescrizione ${ }^{61}$

Riconciliazione terapeutica

La riconciliazione terapeutica è il processo che confronta la lista dei farmaci assunti dal paziente (ricognizione) con quella che dovrebbe essere somministrata in quella particolare circostanza; questo comportamento permette di decidere la terapia giusta da prescrivere

I farmaci conservati in casa

Soprattutto a seguito di recenti cambiamenti della terapia, è comune per le persone anziane e le loro famiglie conservare in casa una scorta di farmaci e continuare a prendere vecchi farmaci insieme a quelli nuovi, con rischio di confusione e di assumere medicamenti scaduti in assenza di supervisione

Farmaci me too

I farmaci me too possono essere definiti come medicamenti chimicamente correlati al prototipo o con identico meccanismo di azione ${ }^{76}$

Questi farmaci (chiamati anche follow-on) in gran parte duplicano l'azione di farmaci già esistenti. Essi mostrano, tuttavia, alcune limitazioni: non presentano nessun vantaggio rispetto ai farmaci già disponibili, riducono l'incentivazione alla ricerca di vere innovazioni, mostrano un inaccettabile rapporto rischio/beneficio e, in quanto più costosi, utilizzano più risorse aumentando la spesa sanitaria

I farmaci me too, dovrebbero sempre essere prescritti in ragione di un esplicito valore aggiunto rispetto ai farmaci di riferimento già consolidati ${ }^{77}$

* Compliance: Descrive il grado con cui il paziente correttamente segue le indicazioni del medico. Generalmente è riferita alla terapia farmacologica prescritta; Aderenza: Descrive come il comportamento del paziente si attiene alle indicazioni terapeutiche concordate con il medico. Tale definizione è stata adottata in alternativa a quella della compliance proprio per sottolineare che il paziente è libero di decidere se aderire alle raccomandazioni del medico e che la mancata decisione di non aderire non dovrebbe essere un motivo per dare la colpa al paziente. L'adesione in sostanza supera il concetto di compliance sottolineando la necessità di un accordo tra medico e paziente; Concordanza: Processo in cui paziente e medico assumono decisioni insieme riguardo al trattamento.

ADRs, adverse drug reactions. 


\section{Eliminare gli sprechi}

Alcuni pazienti ricevono servizi ridondanti e di basso valore. Lo spreco in ambito sanitario è stato definito come qualsiasi spesa finalizzata a produrre servizi che forniscono benefici marginali per la salute, avendo a disposizione alternative meno costose. ${ }^{78}$ Esso è direttamente legato al concetto di incertezza clinica: lo spreco può derivare dalla mancanza di evidenze o quando la diagnosi è incerta, per cui possono essere assunte decisioni diverse nella cura del paziente. Lo spreco clinico si sovrappone spesso a sprechi operativi, ad esempio il mantenimento di servizi inefficienti. L'eliminazione degli sprechi, anche nel contesto del ridotto apporto di finanziamenti all'assistenza sanitaria, può realizzarsi in diversi modi: i) definire accuratamente i livelli di base della sanità pubblica (livelli essenziali di assistenza), selezionando gli obiettivi correlati e concordando costi standard di riferimento; ii) definire le dimensioni ottimali delle organizzazioni sanitarie locali, come ad esempio aree metropolitane estese, concepite come una rete la più larga possibile per erogare servizi sanitari in base ai bisogni reali dei paziente, eliminando funzioni dirigenziali e operative ridondanti; iii) selezionare il numero di letti disponibili negli ospedali e nelle RSA/lungodegenze, secondo le reali esigenze epidemiologiche, considerando l'aumento sostanziale della popolazione anziana; iv) definire i percorsi diagnosticoterapeutici in base alle evidenze, ove possibile; v) inquadrare per ogni paziente cronico le specificità e $\mathrm{i}$ bisogni latenti o manifesti, in modo da consentire ai medici e agli infermieri di prevedere la verosimile traiettoria di malattia; vi) implementare un trattamento il più possibile personalizzato, definendo gli obiettivi terapeutici finali, sulla base di una valutazione multidimensionale del paziente complesso; ${ }^{83}$ vii) individuare i pazienti con una possibile dimissione difficile dall'ospedale, attraverso la valutazione delle loro esigenze (mediche, funzionali, sociali, emotive); viii) ottimizzare il trattamento medico ed assistenziale, con servizi integrati e coordinati, monitorando l'evoluzione delle malattie croniche, anticipando la gestione dei segni di deterioramento; ix) lottare contro gli eccessi di trattamento e gli errori nei processi di cura mediante audit clinico-organizzativi, semplificare la complessità amministrativa e la burocrazia, rendere trasparenti ed omogenei i prezzi con standard di riferimento, eliminare possibili frodi e abusi e qualsiasi altra fonte di inefficienza $^{84,85}$ (Tabella 8); $\mathrm{x}$ ) ridurre o eliminare i servizi di basso valore, intesi come interventi: i) per cui i rischi risultanti probabilmente superano i benefici; ii) che esistono prevalentemente per ragioni auto-referenziali.

Un sistema di rimborso legato all'efficacia dei servizi potrebbe, in quanto in grado di fornire valore clinico aggiunto ai bisogni del paziente, migliorare potenzialmente le cure, riducendo i costi, ${ }^{86}$ con conseguenze significative sulla politica sanitaria e l'organizzazione dei servizi. ${ }^{87} \mathrm{Vi}$ è la necessità di un riordinamento sostanziale degli obiettivi e delle priorità del nostro sistema sanitario pubblico. I medici devono con le loro scelte saper mediare nel potenziale conflitto tra la qualità delle cure, le richieste del singolo paziente e la sostenibilità sociale ed economica dei bisogni della collettività (Tabella 9).

\section{La prescrizione giudiziosa della terapia farmacologica}

La prescrizione giudiziosa della terapia è un prerequisito per l'uso sicuro, appropriato e conveniente dei farmaci. Prima di decidere se un farmaco prescritto

Tabella 6. Le 6 regole di Cochrane per un sistema sanitario frugale ed efficace.

\begin{tabular}{lll}
\hline 1 Considerare solamente tutto ciò che effettivamente funziona & $\begin{array}{l}\text { Confrontare i diversi provvedimenti alternativi e verificare se un tratta- } \\
\text { mento è più nocivo rispetto al fatto di non intervenire }\end{array}$ \\
\hline 2 Mettere a disposizione tutti i possibili trattamenti efficaci & $\begin{array}{l}\text { Essere a conoscenza se sono disponibili gratuitamente trattamenti effi- } \\
\text { caci alternativi per tutti coloro che possono trarne beneficio }\end{array}$ \\
\hline $3 \quad$ Minimizzare i tempi dell'assistenza dovuta & $\begin{array}{l}\text { L'efficacia può essere correlata al tempo necessario per le decisioni cli- } \\
\text { niche, in funzione delle procedure diagnostiche e terapeutiche, dei rela- } \\
\text { tivi tempi e le liste di attesa, i rinvii, la durata di degenza, eccetera }\end{array}$ \\
\hline 4 Trattare i pazienti nel luogo/setting assistenziale più adeguato & $\begin{array}{l}\text { Considerare la migliore collocazione del paziente ai fini della migliore } \\
\text { cura, anche in riferimento alle risorse e ai letti disponibili }\end{array}$ \\
\hline 5 Prevenire solo ciò che è evitabile & $\begin{array}{l}\text { Spendere solo sulla prevenzione realmente efficace } \\
\text { Monitorare solo problemi curabili o prevenibili }\end{array}$ \\
\hline 6 Diagnosticare solo ciò che è curabile & $\begin{array}{l}\text { Ai medici piace fare diagnosi } \\
\text { È questo un approccio sostenibile se nulla si può fare? Questa è una do- } \\
\text { manda importante anche dal punto di vista etico!* }\end{array}$
\end{tabular}

*È evidente che il quesito, pur legittimo (sopratutto alla luce di una crescente medicina difensiva fine a sé stessa) sottende non solamente alcune implicazioni strumentali, ma anche fondamentali quesiti etici, particolarmente nei pazienti fragili, gravati da prognosi incerte nel breve termine o in fase terminale. In base al metodo clinico, prima di poter decidere su un qualsiasi trattamento terapeutico è necessario effettuare una valutazione multidimensionale del paziente e circostanziare la diagnosi, onde poter esprimere un giudizio prognostico e decidere il da farsi. 
è appropriato, in particolare per un paziente anziano, devono essere considerati alcuni principi fondamentali (Tabella 10). ${ }^{89}$

Una proposizione di valutazione del fitness status è stata considerata, ad esempio, per i pazienti affetti da sindromi linfo-proliferative croniche, secondo una classificazione che prevede tre categorie di pazienti (fit, unfit, frail) (Figura 4). ${ }^{90-93}$

L'impiego dei farmaci risulta qualitativamente corretto se corrisponde ad alcuni principi fondanti, descritti dall'Australian National Medicines Policy NPS Medicine Wise: ${ }^{85}$

- uso giudizioso, selezionando saggiamente le opzioni a disposizione: i farmaci prescritti dovrebbero essere utilizzati solo se appropriati, considerando anche alternative non farmacologiche;

- uso appropriato, scegliendo il farmaco più appropriato, tenendo conto della malattia in causa da trattare, i potenziali rischi, i benefici del trattamento, il dosaggio, la durata del trattamento e dei costi;

- uso sicuro, utilizzando i farmaci di comprovata efficacia e sicurezza per ottenere i migliori risultati possibili: l'abuso, l'uso eccessivo o il sottoutilizzo dei farmaci dovrebbero essere ridotti al minimo;

- uso efficace: i medicinali devono raggiungere gli obiettivi terapeutici realizzando espliciti risultati positivi.

Per quanto riguarda l'uso appropriato e ottimale dei farmaci equivalenti, è necessaria una sistematica imponente strategia educativa rivolta ai professionisti della salute e ai pazienti, sostenuta particolarmente dal sistema sanitario nazionale. ${ }^{68}$

Malattie croniche: più auto gestione, condivisione tra diversi professionisti dei dati relativi al paziente, modalità di follow up e diagnosi precoce del peggioramento/riesacerbazione

I programmi di assistenza finalizzati ad assicurare il coordinamento delle cure possono ridurre l'uso non necessario di servizi sanitari e migliorare l'outcome dei pazienti. In particolare i pazienti che transitano da un setting assistenziale ad un altro (transitional care) devono essere supportati da un sistema che garantisca loro una continuità assistenziale, particolarmente nel momento della dimissione dall'ospedale a casa. ${ }^{94}$ Nella fase di passaggio da un contesto di cura ad un altro le possibilità di disfunzione della comunicazione e di errore sono elevati, con possibili conseguenti alti costi, in particolare per possibili richieste di accertamenti, visite specialistiche e/o ricoveri non programmati. ${ }^{95}$ Gli elementi fondamentali per il disease management delle malattie croniche e per gestire al meglio la transizione delle cure sono schematizzati nelle Figure 6 e 7. ${ }^{\mathrm{a}}$

È necessario definire esattamente la tassonomia della gestione delle malattie croniche. ${ }^{96}$ I programmi che educano i pazienti all'autogestione delle malattie croniche sono molto efficaci nel migliorare gli esiti clinici nelle cure primarie. ${ }^{97} \mathrm{La}$ trasmissione delle informazioni sanitarie aggiornate riguardo al paziente, mediante una cartella clinica condivisa tra $\mathrm{i}$ diversi professionisti, consente non solamente di conoscere la storia clinica del paziente, ma soprattutto di evitare omissioni, ripetizioni, ridondanze, errori ed eventi indesiderati. I registri elettronici dei dati clinici condivisi nell'équipe informale tra diversi professionisti che si concentra (proprio con uno strumento condiviso di reciproca comunicazione) sono utili per valutare la qualità del lavoro dei professionisti. Perciò è necessario attivare e implementare una rete, auspicabilmente omogenea su tutto il territorio nazionale, che possa garantire un'adeguata comunicazione interprofessionale su pazienti complessi condivisi. ${ }^{98}$ Il precoce riconoscimento dei sintomi del deterioramento clinico, con un il conseguente intervento tempestivo in malattie croniche o gravi, potrebbe ridurre le riammissioni ospedaliere non programmate, riducendo gli accessi in ospedale e le spese determinate da ricoveri non necessari. In sostanza, qualsiasi piano di gestione integrata delle ma-

a La rete integrata può essere intesa schematicamente in due accezioni:

- Il concetto di rete clinica integrata: l'assistenza sanitaria complessiva viene prodotta e distribuita da una rete di interconnessione tra équipes multi-professionali privilegiando nell'organizzazione le maglie rispetto ai nodi. Un elemento programmatorio fondamentale è la gestione delle relazioni e non la centralizzazione della produzione: non è necessaria una gerarchia tra gli attori-erogatori di assistenza. L'integrazione tra i diversi erogatori pone l'accento sulla necessità di un governo del sistema contro i rischi di diluizione, duplicazione e dispersione delle attività.

- Il concetto di rete gerarchicamente integrata: i) per i servizi afferenti alla medesima linea di produzione l'integrazione a rete può essere realizzata attraverso un modello hub and spoke. Esso prevede la concentrazione della produzione della assistenza di maggiore complessità in centri di eccellenza (hub) e l'organizzazione del sistema di invio da centri periferici funzionalmente sottoordinati (spoke) cui compete principalmente la selezione e l'invio dei pazienti al centro di riferimento; ii) l'adozione del concetto di rete integrata per la programmazione della rete dei servizi rende incompatibile l'uso di un regime di competizione fra le singole unità produttive. Ad esse infatti viene richiesta reciproca cooperazione all'interno della rete di cui fanno parte, secondo i diversi livelli di complessità assistenziale di loro specifica competenza. L'adozione di un modello basato sulla cooperazione funzionale tra $\mathrm{i}$ diversi erogatori consente una utilizzazione della risorsa umana e professionale fortemente indirizzata alla integrazione e al lavoro in team, irrealizzabile in un sistema competitivo. 
lattie deve essere basato su una stretta collaborazione tra l'ospedale e il territorio.

\section{L'assistenza sanitaria domiciliare nurse directed per le persone anziane può portare ad una riduzione dei costi?}

I programmi di assistenza domiciliare per gli anziani svolti da infermieri ed altri operatori sanitari, influenzano positivamente lo stato funzionale e potrebbero promuovere alcuni vantaggi clinici. Tuttavia, in generale, non è ancora chiaro quali componenti e modalità di tali interventi possano contribuire a specifici aspetti di assistenza, né è definito se gli interventi home-based possano offrire un vantaggioso rapporto costo/beneficio per il servizio sanitario nazionale. ${ }^{99}$

\section{L'inerzia professionale può condizionare i costi dell'assistenza sanitaria}

L'inerzia in medicina è stata definita come l'incapacità dei professionisti ad assumere decisioni necessarie (ad esempio, nella fase di accertamento della diagnosi - latenza diagnostica) o ad iniziare/intensificare $\mathrm{i}$ trattamenti, quando indicati, in maniera tempestiva, ${ }^{100,101}$ anche in presenza di linee guida disponibili. ${ }^{102}$ L'inerzia decisionale non riguarda sola- mente il medico direttamente responsabile del paziente, ma anche gli specialisti consultati. Essa può derivare anche dall'atteggiamento del paziente e della sua famiglia alle cure, nonché dal contesto organizzativo (Tabella 11). ${ }^{103-105}$

\section{Incertezza, medicina basata sull'evidenza e il processo decisionale in Medicina Interna}

L'internista assume le sue decisioni in tre tipi di situazioni: i) certezza: viene adottata la decisione ideale ed eseguita la strategia corrispondente; ii) rischio: la più adatta alternativa viene selezionata in base a criteri predittivi o di probabilità pre-test; iii) incertezza, in cui le decisioni sono legate a tre diversi fattori (teoria del triplice agente): l'opinione (e i valori) del paziente, del contesto sociale e del medico. ${ }^{106}$

L'incertezza, che in alcuni casi diventa una forte barriera decisionale, può essere legata ad aspetti cognitivi (scarse conoscenze), a carenza di abilità, ai comportamenti (ad esempio, paura di azioni legali) o ad aspetti affettivi (ansia decisionale). Gli internisti hanno caratteristiche proprie, fondate principalmente sulla capacità di giudizio clinico, che comprende il ragionamento clinico in merito alle reali esigenze del paziente, il pensiero critico e la comprensione globale della situazione unita alle competenze acquisite. La frequente incertezza che grava sui processi decisionali

Tabella 7. Alcune possibili strategie per un'assistenza sanitaria di (dis)investimento costruttiva e saggia.

Privilegiare sempre la prevenzione, come strumento fondamentale per mantenere un sistema sanitario efficace e sostenibile

Assumere le migliori decisioni basate sull'evidenza (quando possibile)

Migliorare il coordinamento dei servizi tra i fornitori di cure primarie e secondarie e la continuità delle cure nelle malattie croniche

Ridurre le ospedalizzazioni evitabili mediante un rafforzamento delle cure primarie, con analisi esplicita dei risultati ottenuti

Individuare precocemente i sintomi di peggioramento nei pazienti con malattie croniche, per prevenire riammissioni non programmate in ospedale

Migliorare l'integrazione del sistema sanitario con i servizi sociali e assistenziali locali

Considerare quale effettiva rilevanza clinica richiesta agli studi clinici e coinvolgere i comitati etici per l'analisi critica dei risultati

Valutare l'utilità, l'allocazione e la distribuzione delle nuove tecnologie sanitarie ed organizzative proposte mediante l'HTA, prima di acquisire nuove tecnologie o procedere a cambiamenti organizzativi radicali dell'ospedale

Accettare i cambiamenti nella pratica abituale di lavoro, quando documentata da esplicite analisi di costi/benefici

Razionalizzare il numero e la distribuzione di tecnologie ad alto costo in base al bacino di utenza

Assegnare le risorse in aree omogenee secondo principi di equità e trasparenza nella valutazione dei carichi di lavoro senza conflitto di interesse

Ridurre qualsiasi conflitto d'interesse professionale e/o corporativo nella cura dei pazienti, evitando autoreferenziali nicchie di privilegio

Collaborare (piuttosto che competere) tra professionisti afferenti allo stesso ambito organizzativo su obiettivi espliciti e trasparenti senza conflitto di interesse

Facilitare la legittimazione dei pazienti a divenire protagonisti attivi nella gestione della loro salute ed assistenza sanitaria, in particolare per l'attuazione del autogestione delle malattie croniche

Incentivare programmi di sviluppo e di mantenimento delle competenze cliniche dei professionisti

Ridurre i costi amministrativi e la burocrazia

Implementare setting assistenziali finalizzati alle cure palliative (non solo per i pazienti oncologici), in alternativa ai ricoveri ospedalieri

HTA, Health Technology Assessment. 
nei complessi pazienti ricoverati in Medicina interna richiede una riflessione critica sugli studi clinici, su cui si fonda la medicina basata sull'evidenza, sia per i criteri di selezione dei casi inclusi/esclusi, sia in termini di metodologia di valutazione dei risultati. Nell'analisi degli esiti dei trials clinici dovrebbero essere presi in considerazione non solo la significatività statistica dei risultati, ma anche la loro rilevanza clinica, in base al principio di minimal clinical important difference (MCID) ${ }^{107}$ Considerare la MCID è fondamentale nelle decisioni terapeutiche anche ai fini dell'analisi dei costi/benefici, ad esempio, nell'implementazione di farmaci molto costosi, come quelli per i tumori. Sulla base di una valutazione multidimensionale, possiamo pertanto decidere quale tipo di intervento offrire ai nostri pazienti, in termini di livello di cura, intensiva, interventistica, conservativa o palliativa. Esplorando la vera complessità dei nostri pazienti e selezionando le loro reali esigenze, siamo in grado di esercitare un'assistenza sanitaria olistica, individuale, antropologica, ovvero di esercitare appieno i principi della Medicina Interna.

\section{I medici devono condividere le decisioni con i pazienti e non soddisfare incondizionatamente alcune delle loro ingiustificabili richieste}

L'evidence-based medicine prende in considerazione le preferenze dei paziente nelle decisioni cliniche. Coinvolgere i pazienti nella loro cura è importante, ad esempio nella definizione dei patient reported outcome (PRO), letteralmente traducibile come risultati riportati dai pazienti, processo auspicabile ma non sempre applicabile. [I PRO permettono di descrivere e valutare lo stato di salute del paziente attraverso la sua stessa percezione della propria condizione di salute.] Condividere le decisioni può promuovere la scelta di terapie meno costose

Tabella 8. Gli sprechi in sanità: possibili fattori coinvolti.

\begin{tabular}{|c|c|c|c|c|c|c|}
\hline $\begin{array}{l}\text { Esempi/ } \\
\text { commenti }\end{array}$ & Overtreatment & $\begin{array}{l}\text { Inadeguato } \\
\text { coordinamento } \\
\text { delle cure }\end{array}$ & $\begin{array}{l}\text { Processo di cura } \\
\text { non adeguato }\end{array}$ & $\begin{array}{l}\text { Eccessiva } \\
\text { burocrazia e } \\
\text { complessità } \\
\text { amministrativa }\end{array}$ & $\begin{array}{l}\text { Prezzi non } \\
\text { trasparenti }\end{array}$ & Frodi e abusi \\
\hline & $\begin{array}{l}\text { Eccessivo uso di } \\
\text { antibiotici, } \\
\text { ricorso alla } \\
\text { chirurgia quando } \\
\text { sarebbe meglio } \\
\text { vigilare e } \\
\text { aspettare, cure } \\
\text { intensive alla fine } \\
\text { della vita per } \\
\text { pazienti che } \\
\text { preferirebbero } \\
\text { cure al proprio } \\
\text { domicilio o in } \\
\text { hospice }\end{array}$ & $\begin{array}{l}\text { Cure } \\
\text { frammentate, } \\
\text { principalmente } \\
\text { nelle malattie } \\
\text { croniche, a fronte } \\
\text { a complicazioni, } \\
\text { ricoveri } \\
\text { ospedalieri } \\
\text { inattesi, } \\
\text { peggioramento } \\
\text { dell'autonomia } \\
\text { funzionale }\end{array}$ & $\begin{array}{l}\text { Esecuzione } \\
\text { carente/mancata } \\
\text { adesione ai } \\
\text { migliori processi } \\
\text { di cura, } \\
\text { insufficiente } \\
\text { sicurezza e scarsa } \\
\text { prevenzione con } \\
\text { esiti negativi e } \\
\text { danni } \\
\text { indesiderati nel } \\
\text { paziente }\end{array}$ & $\begin{array}{l}\text { Fallimento nella } \\
\text { standardizza- } \\
\text { zione di regole } \\
\text { finalizzate ad } \\
\text { eliminare proce- } \\
\text { dure ridondanti e } \\
\text { sbagliate e a } \\
\text { ridurre le spese } \\
\text { amministrative } \\
\text { incongrue }\end{array}$ & $\begin{array}{l}\text { Assenza di } \\
\text { trasparenza in un } \\
\text { mercato } \\
\text { competitivo }\end{array}$ & $\begin{array}{l}\text { I comportamenti } \\
\text { professionali } \\
\text { immorali di } \\
\text { poche persone } \\
\text { possono inficiare } \\
\text { gravemente tutto } \\
\text { il sistema }\end{array}$ \\
\hline $\begin{array}{l}\text { Stima della } \\
\text { spesa per gli } \\
\text { sprechi } \\
\text { (anno } 2011 \text { in } \\
\text { miliardi di \$) }\end{array}$ & $158-226$ & $25-45$ & $102-154$ & $107-389$ & $84-178$ & $82-272$ \\
\hline
\end{tabular}

Adattata da Berwick and Hackbarth, 2012. ${ }^{84}$

Tabella 9. Elementi fondamentali per una medicina sostenibile.

\begin{tabular}{ll}
\hline L'assistenza sanitaria deve essere economicamente sostenibile & Ciò comporta il controllo dei costi commisurato alla qualità delle cure \\
\hline $\begin{array}{l}\text { L'assistenza sanitaria sostenibile richiede un rapporto adeguato } \\
\text { tra paziente e medico, anche in riferimento alla sostenibilità } \\
\text { economica }\end{array}$ & $\begin{array}{l}\text { Una buona relazione tra medico e paziente è un prerequisito per facilitare } \\
\text { una migliore adesione alle cure, un minor ricorso ad altri medici specialisti } \\
\text { e ai servizi di emergenza }\end{array}$ \\
\hline $\begin{array}{l}\text { L'assistenza sanitaria sostenibile deve perseguire l'obiettivo } \\
\text { di lungo termine di una massimizzazione degli obiettivi di }\end{array}$ & $\begin{array}{l}\text { È necessario diffondere la cultura di uno stile di vita e di un'alimentazione } \\
\text { salutari, dell'esercizio fisico e della realizzazione personale }\end{array}$
\end{tabular}
salute nella società

Adattata da Kimberton Clinic. ${ }^{88}$ 
ugualmente efficaci, ma con un vantaggio che può arrivare fino ad un $20 \%$ del contenimento dei costi. Tale condivisione dovrebbe presupporre la discussione delle opzioni terapeutiche in un linguaggio comprensibile, con la descrizione delle conoscenze e degli studi disponibili e dei reali obiettivi clinici da raggiungere, illustrando i potenziali effetti collaterali e le complicazioni. ${ }^{108}$ Diversi fattori possono interferire in questo processo, oltre alla malattia di base: gli effetti psicodinamici della malattia, la depressione, la disfunzione cognitiva, i fattori sociali ed economici, la solitudine, eccetera, rendendo il normale coinvolgimento del paziente molto difficile. I pazienti spesso esprimono preferenze su certi accer-

Tabella 10. Decidere se un farmaco prescritto è appropriato.

Considerazioni $^{87}$

- Ragionare oltre i farmaci, considerando la terapia non farmacologica, le cause sottese potenzialmente trattabili e la prevenzione

- Rinviare ogni trattamento farmacologico non immediatamente necessario

- Fare attenzione all'impiego di farmaci di non comprovata efficacia e dei nuovi farmaci

- Essere scettici sui farmaci che hanno evidenziato risultati surrogati

- Considerare i possibili bias di pubblicazione sugli esiti dei trials clinici

- Iniziare il trattamento con un solo nuovo farmaco alla volta

- Bilanciare i rischi potenziali di un farmaco rispetto ai suoi benefici

- Negli anziani: cominciare la terapia lentamente, andare piano, controllare molto attentamente

- Mantenere una vigilanza attiva sugli effetti indesiderati della terapia farmacologica

- Essere consapevoli delle sindromi da sospensione della terapia

- Educare i pazienti a riconoscere le reazioni avverse

- Evitare le terapie in assenza di indicazioni provate

- Concordare il trattamento con i pazienti secondo un programma comune

- Considerare la non aderenza prima di aggiungere farmaci nuovi

- Sospendere la terapia risultata non necessaria dopo la riconciliazione terapeutica

- Ascoltare e rispettare le opinioni del paziente circa l'uso di farmaci

\section{Quesiti $^{58}$}

- Il farmaco è realmente necessario?

- Sono disponibili farmaci equivalenti?

- Ci sono sovrapposizioni inutili nella terapia in corso?

- Le dosi giornaliere, la frequenza e l'intervallo delle somministrazioni sono compatibili con le caratteristiche farmacocinetiche/farmacodinamiche del farmaco?

- Le dosi giornaliere, la frequenza e l'intervallo delle somministrazioni sono compatibili con le caratteristiche del paziente, le comorbilità e potenziali interazioni tra farmaci?

- La terapia prescritta è on/off label?

- La terapia prescritta è congruente con le linee guida più aggiornate sul problema da trattare?

- La terapia prescritta è compatibile con le politiche locali finalizzate ad una buona pratica clinica?

- Il farmaco prescritto è quello più vantaggioso dal punto di vista economico?

- Nel trattamento sono stati omessi o tralasciati farmaci effettivamente necessari?

- Nel trattamento dei pazienti con cancro siamo sicuri che la terapia prescritta (spesso molto costosa) sia adeguata alle caratteristiche del paziente, in base ad un giudizio multidimensionale di robustezza (fitness) o fragilità (frailty) dell'ammalato? (Figure 4 e 5$)^{20}$

- Quale valore aggiunto, clinicamente significativo, può offrire il farmaco prescritto?

- Gli endpoints dei trattamenti dovrebbero essere basati non solamente sulla valutazione della significatività statistica dei trial clinici, ma anche sulla minima differenza clinicamente significativa che rende ragionevole e clinicamente accettabile l'implementazione e l'impiego nella pratica clinica dei farmaci proposti, soprattutto quando questi sono molto costosi

\begin{abstract}
FIT
Paziente candidato ad un trattamento più aggressivo, mirato ad un potenziale eradicamento della malattia e ad un prolungamento della sopravvivenza

\section{UNFIT}

Paziente candidato ad un trattamento meno aggressivo, mirato all' ottenimento di una risposta ed al prolungamento della PFS progression-free survival

FRAIL

Paziente candidato a cure palliative
\end{abstract}

Figura 4. La valutazione del fitness status del paziente con lymphocytic leukemia..$^{90-93}$

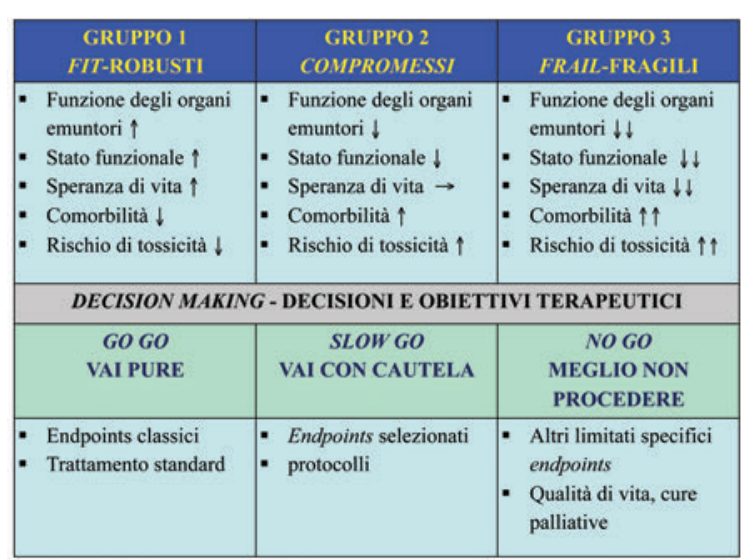

Figura 5. Esempio di decisione basata sulla valutazione del paziente: il malato oncologico anziano. ${ }^{20}$ 
tamenti da effettuare o su trattamenti di loro scelta e i medici, anche se ritengono che tali interventi non siano benefici, possono esserne condizionati. ${ }^{109,110}$ Dare sempre incondizionatamente soddisfazione al paziente non sempre significa raggiungere i migliori risultati. Secondo i dati di uno studio condotto tra il 2000 e il 2007 la maggiore soddisfazione del paziente si associava sì ad un minor numero di visite al pronto soccorso, ma insieme ad un maggiore ricorso al ricovero ospedaliero, ad una spesa superiore per farmaci e sanitaria complessiva e ad aumentata mortalità. ${ }^{111}$ La riduzione delle richieste inappropriate del paziente dovrebbero essere indirizzate ad una migliore comunicazione tra medico e paziente, attraverso l'esplicitazione del percorso diagnosticoterapeutico e del piano di trattamento, finanche al diniego dei desiderata inopportuni, ${ }^{112}$ improntata alla massima trasparenza e col massimo coinvolgimento dei pazienti ai fini di una decisione finale condivisa. ${ }^{113}$ Decisioni condivise facilitano il commitment dei pazienti, promuovono la capacità di autogestione della malattia e migliorano la loro adesione al piano di assistenza globale. ${ }^{114}$

\section{Gli audit clinici come strumento di miglioramento della qualità dell'assistenza}

I medici possono fornire troppe o troppo poche cure, prescrivendo in alcuni casi esami e terapie inutili, in altri percorsi diagnostici e trattamenti insufficienti o errati, con un potenziale impatto negativo sulla qualità dell'assistenza sanitaria. ${ }^{115}$

La verifica e i feedback sulle prestazioni professionali sembrano migliorare la qualità delle cure, soprattutto quando le prestazioni sono scadenti, ma possono portare a importanti miglioramenti nella pra- tica professionale. L'efficacia degli audit sembra dipendere dalla performance di riferimento e da come esso viene effettuato. Il risultato dell'audit sembra essere migliore e più efficace quando le prestazioni di partenza sono mediocri, se effettuato fra pari da un supervisore collega, in più riunioni, se presentato in maniera formale, con obiettivi espliciti ed un piano d'azione di miglioramento. ${ }^{116}$

\section{L'iniziativa della US National Physicians Alliance: Choosing wisely, ovvero scegliere con saggezza}

Nel 2011 è stato pubblicato il lavoro The 'Top 5' Lists in Primary Care: Meeting on the Responsibility of Professionalism, sulla base di un'iniziativa della $U S$ National Physicians Alliance Promoting Good Stewardship in Clinical Practice che, attraverso l'American Board of Internal Medicine (ABIM) ha sviluppato 5 liste top, su tematiche cruciali nelle cure primarie, in medicina interna ed in pediatria, basate sulle evidenze disponibili. Ogni tema era supportato dall'esplicitazione dei benefici per i pazienti, dei potenziali miglioramenti della qualità delle cure e del valore dei servizi forniti, della diminuzione dei rischi e, ove possibile, anche della riduzione dei costi. ${ }^{117-119} \mathrm{Da}$ allora le iniziative di choosing wisely si sono estese ad altre 52 Società Scientifiche ${ }^{120}$ ed altre associazioni. In Italia la Società di Slow Medicine ha introdotto il Progetto Fare di più non significa fare meglio, per un idea di medicina sobria, rispettosa, giusta, ${ }^{121}$ cui anche FADOI ha aderito.

\section{Conclusioni}

La Bioetica ha da tempo affrontato il problema del contenimento dei costi sanitari e delle modalità

\begin{tabular}{|c|c|c|c|c|c|c|}
\hline $\begin{array}{l}\text { Pazienti } \\
\text { in causa }\end{array}$ & $\begin{array}{c}\text { Contesto } \\
\text { di intervento }\end{array}$ & $\begin{array}{c}\text { Team } \\
\text { multidisciplinare } \\
\text { coinvolto }\end{array}$ & $\begin{array}{c}\text { Metodi } \\
\text { di comunicazione }\end{array}$ & $\begin{array}{c}\text { Intensità } \\
\text { assistenziale }\end{array}$ & $\begin{array}{c}\text { Setting } \\
\text { assistenziale }\end{array}$ & Indicatori \\
\hline $\begin{array}{l}\text { - Condizione } \\
\text { di rischio } \\
\text { - Comorbilità } \\
\text { associate } \\
\text { - Caratteristiche } \\
\text { non cliniche } \\
\text { del paziente }\end{array}$ & $\begin{array}{l}\text { - Relazione } \\
\text { pazienteléquipe } \\
\text { curante } \\
\text { - Relazione } \\
\text { pazientel } \\
\text { caregiver } \\
\text { - Livello culturale } \\
\text { di paziente } \\
\text { e caregiver } \\
\text { - Supporto } \\
\text { parentale- } \\
\text { amicale } \\
\text { - Monitoraggio } \\
\text { a distanza }\end{array}$ & $\begin{array}{l}\text { - Medici } \\
\text { - Infermieri } \\
\text { - Assistente } \\
\text { sociale } \\
\text { - Dietista } \\
\text { - Terapisti } \\
\text { - Psicologi } \\
\text { - Farmacista } \\
\text { - Case manager } \\
\text { - Coordinatori } \\
\text { del caso }\end{array}$ & $\begin{array}{l}\text { - Diretto } \\
\text { (face to face) } \\
\text { - Per gruppi } \\
\text { - Telefonico } \\
\text { - Internet } \\
\text { - Telemetria }\end{array}$ & $\begin{array}{l}\text { - Modalità } \\
\text { di accesso } \\
\text { - Durata della } \\
\text { presa in carico } \\
\text { - Frequenza } \\
\text { dei controlli } \\
\text { - Complessità } \\
\text { dei controlli }\end{array}$ & $\begin{array}{l}\text { - Ospedaliero } \\
\text { - Ambulatoriale } \\
\text { - Domiciliare }\end{array}$ & $\begin{array}{l}\text { - Esito clinico } \\
\text { di processo } \\
\text { - Qualità di vita } \\
\text { - Soddisfazione } \\
\text { del paziente } \\
\text { - Soddisfazione } \\
\text { del provider }\end{array}$ \\
\hline
\end{tabular}

Figura 6. Fattori da considerare nel disease management delle malattie croniche. Fonte: Krumholz et al., $2006 .{ }^{96}$ 
di allocazione delle risorse limitate ai fini della garanzia di una giustizia distributiva. Attualmente va introdotto un altro principio ugualmente etico, focalizzato sul come evitare gli sprechi nell'assistenza sanitaria, con evidenti importanti implicazioni economiche per l'organizzazione di servizi. Crediamo fermamente che un'agenda comune condivisa fra Medicina Interna e cittadini, professionisti e decisori nella pianificazione delle cure, possa essere una grande opportunità per qualsiasi cambiamento in ambito sanitario, con un esplicito obiettivo di mantenere e garantire la salvaguardia del sistema pubblico. Il futuro del sistema sanitario non può limitarsi alla riduzione dei costi, ma dovrebbe mirare a fornire una migliore assistenza sanitaria in relazione al denaro speso. Per affrontare questa sfida il ruolo del medico è fondamentale. ${ }^{122} \mathrm{Il}$ medico sta al capezzale del malato, è depositario della responsabilità della salute e

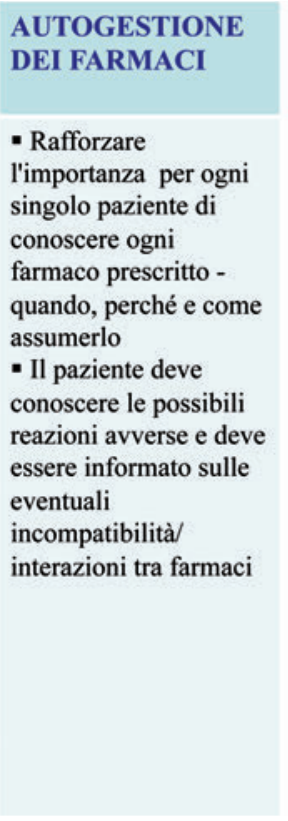

CARTELLA
SANITARIA
PERSONALE
- Anamnesi clinica del
paziente
- Evidenza dei segni
premonitori e dei
sintomi di allarme (red
flags) di
peggioramento della
malattia
- Elenco aggiornato dei
farmaci assunti e delle
allergie
- Check-list strutturata
delle modalità di
controllo e visite
successive
- Evidenza dei dubbi,
preoccupazioni,
desideri espressi del
paziente

\begin{tabular}{l} 
MODALITÀ DI \\
FOLLOW-UP \\
\hline - Percorso diagnostico- \\
terapeutico ed \\
assistenziale condiviso \\
nel contesto locale \\
- Coinvolgere il \\
paziente nel \\
programma di cura, \\
specificando le \\
modalità dei controlli \\
successivi, le ulteriori \\
visite/indagini \\
necessarie, \\
eventualmente \\
provvedendo \\
direttamente alla \\
pianificazione e \\
prenotazione del \\
follow-up \\
- Utilizzare la rete \\
delle cure per patologia \\
croniche specifiche
\end{tabular}

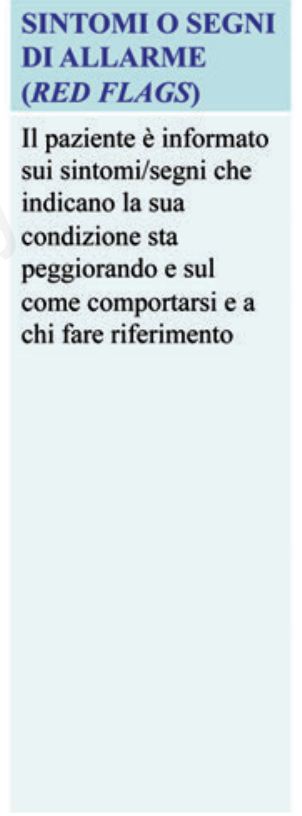

Figura 7. I 4 pilastri fondamentali della transitional care.

Tabella 11. Fattori che contribuiscono all'inerzia decisionale clinica e/o prescrittiva.

\begin{tabular}{|c|c|c|}
\hline Medico & Paziente & Sistema sanitario \\
\hline $\begin{array}{l}\text { - } \text { Competenza ed abilità } \\
\text { - } \text { correnti } \\
\text { - Ritardo avvio del trattamento } \\
\text { - Difficoltà ad individuare obiettivi di cura } \\
\text { chiari } \\
\text { - Trattamento non congruente agli obiettivi } \\
\text { - terapeutici } \\
\text { - Difficoltà a gestire le comorbilità } \\
\text { - Incapacità a selezionare le priorità } \\
\text { - Sottovalutazione dei bisogni dei pazienti } \\
\text { - Temrastima di problemi non prioritari } \\
\text { - Trattamento sintomatico piuttosto che } \\
\text { - } \text { proattivo } \\
\text { - dimore di ripercussioni legali, medicina } \\
\text { - Comporiva } \\
\text { sistema dei DRGs in ambito ospedaliero }\end{array}$ & $\begin{array}{l}\text { - Reazioni avverse ai farmaci } \\
\text { - Politerapia } \\
\text { - Aderenza } \\
\text { - Costo dei farmaci } \\
\text { - Negazione della malattia e/o della sua } \\
\text { gravità } \\
\text { - Assenza di sintomi } \\
\text { - Paura delle conseguenze delle malattie } \\
\text { - Approccio fatalistico } \\
\text { - Scarsa comunicazione con il medico } \\
\text { - Deterioramento cognitivo, depressione, } \\
\text { - } \text { malattia mentale, abuso di sostanze } \\
\text { - Pressione di vita } \\
\text { medico }\end{array}$ & $\begin{array}{l}\text { - Influenza dell'industria farmaceutica } \\
\text { - Influenza dei colleghi e degli stakeholders } \\
\text { - Influenza dei mezzi di comunicazione } \\
\text { - Assenza di linee guida cliniche } \\
\text { - Nessuna pianificazione di follow-up medico } \\
\text { - Mancata educazione del paziente sulla } \\
\text { - Troppi medici/sub-specialisti nella cura } \\
\text { - dello stesso paziente } \\
\text { - Mancanza di coordinamento } \\
\text { - } \text { - Marsa comunicazione tra i medici e altri } \\
\text { Mancanza di risorse pratiche }\end{array}$ \\
\hline
\end{tabular}

DRGs, diagnosis-related groups. 
della vita che il paziente gli affida, e sulla base della diagnosi clinica pianifica la strategia ed attua le cure. ${ }^{123,124}$ Anche in questo periodo di austerità possono ancora essere trovate nuove opportunità e i medici devono continuare a sforzarsi per affrontare questa sfida. ${ }^{125}$ I dottori non possono esimersi dal responsabilizzarsi rispetto all'aumento dei costi sanitari. Il modello basato sulla top five lists ha il vantaggio di definire le cause più comuni di spreco e di futilità nella pratica medica, dimostrando che $\mathrm{i}$ medici stanno realmente tutelando gli interessi dei pazienti e non semplicemente il razionamento delle cure. I medici devono riconsiderare le loro scelte nella cura dei pazienti, per un'assistenza di qualità che tenga conto dei costi dei servizi forniti, ${ }^{126,127}$ ricordando non è sempre vero che il più è il meglio. $\mathrm{Al}$ contrario, dobbiamo rafforzare il concetto che il meno può essere meglio, ${ }^{128}$ diffondendo il principio della migliore assistenza a costi minori. ${ }^{129}$ Nella nostra Società scientifica di Medicina Interna (la Federazione delle Associazioni dei Dirigenti Ospedalieri Internisti, FADOI), vogliamo sostenere l'opinione che un'assistenza sanitaria essenziale è ancora un obiettivo da raggiungere in tutti gli ospedali, non solo in Italia, ma in tutto il mondo. Siamo alla ricerca di modelli originali di un approccio sostenibile nei pazienti di Medicina Interna, ribadendo i principi fondanti di metodologia clinica per i nostri pazienti. Vorremmo offrire alcune idee finalizzate ad un approccio congruente con le istanze economiche attuali in Medicina Interna, proponendo alcune raccomandazioni - già pubblicate ${ }^{130} \mathrm{e}$ disponibili anche in questo numero monografico in italiano. Una pratica clinica basata su interventi medici utili, selettivi, efficaci e saggi, attraverso il ruolo attivo e propositivo dei medici, può consentire di selezionare le priorità ed identificare il più importante dei problemi complessi. Dobbiamo imparare (o re-imparare) a praticare una medicina basata sull'anamnesi e l'esame obiettivo, sulla relazione sensoriale con i nostri pazienti, più basata sull'ascolto e l'osservazione (high listening and watching), nonché su un contatto fisico con il paziente (high touching), piuttosto che su una delega rimessa (anche per le responsabilità professionali) ad accertamenti laboratoristico-strumentali. La competenza del medico internista cioè dovrebbe basarsi su un'interazione clinica globale con il paziente, non così sempre dipendente dalla tecnologia (high tech). ${ }^{131}$

Ribadiamo, in sostanza, gli elementi salienti già esplicitati nella originale declinazione della mission della FADOI:

- Siamo convinti che la buona metodologia clinica resta un valido strumento per la crescita razionale ed equilibrata degli ospedali.

- Sostituire il ragionamento clinico con l'immediato ricorso alla diagnosi strumentale vuol dire lasciar crescere una visione fortemente tecnologica della medicina, ovvero:

- Costruire una medicina insostenibile, sia dal punto di vista organizzativo che sul piano economico.

In questo modo, sarà più facile per noi ricordare, come ci insegnarono i nostri Maestri, che la diagnosi è basata, nella maggior parte dei casi, sull'anamnesi clinica e l'esame fisico e che l'ultimo farmaco introdotto in commercio non è necessariamente il migliore.

\section{Bibliografia}

1. ISTAT (Italian Institute of Statistics). Noi Italia. 100 statistiche per capire il Paese in cui viviamo. Available from: http://noi-italia.istat.it [In Italian].

2. Rich G, Leonard P, Zalmanovitch Y, Vashdi D. Can we make cuts that will not harm health care? BMJ 2010; 340:628-31.

3. Lowes R. Decide by Dec. 31 whether to stay in medicare, societies say. Medscape Medical News, Dec 27, 2012. Available from: http://www.medscape.com/ viewarticle $/ 776778$

4. Iglehart JK. Expanding eligibility, cutting costs - A Medicaid Update. NEJM 2012;366:105-7.

5. Hussey PS, Wertheimer S, Mehrotra A. The association between health care quality and cost: a systematic review. Ann Intern Med 2013;158:27-34.

6. Santacroce G. Judicial intervention in the medical profession: present and future regulation of disputes concerning professional liability. Ital J Med 2012;6:253-4.

7. Studdert DM, Mello MM, Sage WM, et al. Defensive medicine among high risk specialist physicians in a volatile malpractice environment. JAMA 2005;293: 2609-17.

8. Hettrich CM, Mather RC $3^{\text {rd }}$, Sethi MK, et al. The costs of defensive medicine. AAOS Now, Dec 2010. Available from: http://www.aaos.org/news/aaosnow/dec10/ advocacy2.asp

9. Emanuel E, Tanden N, Altman S, et al. A systemic approach to containing health care spending. NEJM 2012;367:949-54.

10. Bishop TF, Federman AD, Keyhani S. Physicians' views on defensive medicine: a national survey. Arch Intern Med 2010;170:1081-3.

11. Hatch SO. Invited commentary-it is time to address the costs of defensive medicine: comment on "physicians' views on defensive medicine: a national survey". Arch Intern Med 2010;170:1083-4.

12. Orsini E. The appropriateness in medicine: an essential condition for the quality and the efficiency of health care. Cardiologia Ambulatoriale 2012;20:232-9.

13. Sen AK. The impossibility of a paretian liberal. J Polit Econ1970;78:152-7.

14. The Agency for Healthcare Research and Quality and The Employer Health Care Alliance Cooperative. Highlights from a National Conference, Appendix A. Last update: of 11/06/2006. Available from: http://www. academyhealth.org/files/publications/EfficiencyReport.pdf

15. Cislaghi C. Economia dei farmaci: valutazione di effi- 
cienza, governo della distribuzione, governo della domanda. Roma: Age.Na.S (Agenzia Nazionale per i Servizi Regionali); 2005. Available from: http://www. agenas.it/ppt/4_Economia_dei_farmaci.ppt

16. Fuchs VR. The doctor's dilemma - what is "appropriate" care? NEJM 2011;365:585-7.

17. Gabbay E, Calvo-Broce J, Meyer KB, et al. The empirical basis for determinations of medical futility. J Gen Intern Med 2010;25:1083-9.

18. Schneiderman LJ, Jecker NS, Jonsen AR. Medical futility: its meaning and ethical implications. Ann Intern Med 1990;112:949-54.

19. Moratti S. The development of "medical futility": towards a procedural approach based on the role of the medical profession. Med Ethics 2009;35:369-72.

20. Wedding U. Endpoints and their relevance to older people, cancer and palliative care and work of EORTC. Elderly Task Force EORTC. Brussels: University of Jena; 2012.

21. Reich J. A critical appraisal of overdiagnosis: estimates of its magnitude and implications for lung cancer screening. Thorax 2008;63:377-83.

22. Welch G, Schwartz L, Woloshin S. Overdiagnosed: making people sick in pursuit of health. Boston, MA: Beacon Press; 2011.

23. Moynihan R, Cassels A. Selling sickness: how the world's biggest pharmaceutical companies are turning us all into patients. New York, NY: Nation Books; 2005.

24. Hoffman JR, Cooper RJ. Overdiagnosis of disease: a modern epidemic. Arch Intern Med 2012;172:1123-4.

25. Moynihan R, Heath I, Henry D. Selling sickness: the pharmaceutical industry and disease mongering. BMJ 2002;324:886-91.

26. Special Collection on Disease Mongering. Public Library of Science (PloS Medicine). Available from: http:// www.ploscollections.org/article/browseIssue.action?issue =info $\% 3$ Adoi $\% 2 F 10.1371 \% 2$ Fissue.pcol.v07.i02

27. Nardi R, Gardellini A, Iori I. Internal Medicine wards overcrowding and clinical risk management: structural or systemic interventions needed? Ital J Med 2009;3:3-8.

28. Heneghan C. General practice, p 623. In: Cook S. Experts' guide to saving money in health. BMJ 2010; 340:c1281.

29. Ministero della Salute. Stato di salute e prestazioni sanitarie nella popolazione anziana - anno 2000. Rome: Italian Ministry of Health; 2003. Available from: http://www.ministerosalute.it/pubblicazioni/ppRisultato.jsp?id=379 [In Italian].

30. Cioni G. Indici di occupazione, degenza media, sovraffollamento dei reparti internistici - Fattori determinanti: il punto di vista dell'internista. In: Attualità e prospettive della Medicina Interna nel Servizio Sanitario Nazionale, Bologna 14 aprile 2011. Comunicazione orale, in italiano.

31. George G, Jell C, Todd BS. Effect of population ageing on emergency department speed and efficiency: a historical perspective from a district general hospital in the UK. Emerg Med J 2006;23:379-83.

32. Di Lenarda A, Scherillo M, Maggioni AP, et al. Current presentation and management of heart failure in cardiology and internal medicine hospital units: a tale of two worlds. The TEMISTOCLE study. Am Heart J 2003; 146:E12.

33. Biagi P. The CONFINE (Comorbidities and Outcome
iN patients with chronic heart Failure: a study in INternal mEdicine units) study: a new epidemiologic observational study on heart failure in the internal medicine departments in Italy. Ital J Med 2007;1:57-64.

34. Biagi P, Gussoni G, Iori I, et al. Clinical profile and predictors of in-hospital outome in patients with heart failure: The FADOI "CONFINE" Affiliations. Int H Cardiol 2011;152:88-94.

35. Zapatero A, Barba R, Marco J, et al. Predictive model of readmission to internal medicine wards. Eur J Intern Med 2012;23:451-6.

36. Tellini M, Petrioli A, Forni S, Morettini A. The Revolving Door Syndrome in Internal Medicine. A study on 11.846 subjects discharged from all Internal Medicine departments of Tuscany with diagnosis of heart failure and pneumonia. Ital J Med 2014 [in press].

37. Ludke RL, Wakefield DS, Booth BM, et al. Appropriateness of admissions and discharges among readmitted/patients. Health Service Res 1993:3;501-25.

38. Karen EJ, Jha AK. Thirty-day readmissions - truth and consequences. N Engl J Med 2012;366:1366-9.

39. Kassirer JP, Milstein A. Our failure to curb excessive testing. Arch Intern Med 2012;172:1751-2.

40. Australian National Medicines Policy-NPS MedicineWise. Available from: http://www.nps.org.au/publications/health-professional/prescribing-practice-review

41. Taylor R. What is health technology assessment? April 2009. Available from: http://www.medicine.ox.ac.uk/ bandolier/painres/download/whatis/What_is_health_ tech.pdf

42. Ghiddi L. Diagnostica per immagini: facciamo ordine: appropriatezza prescrittiva, responsabilità, sostenibilità. Presentazione al Seminario "L'appropriatezza diagnostica, terapeutica e organizzativa in area medica: l'impegno dei professionisti delle Aziende Sanitarie della provincia di Reggio Emilia”, Reggio Emilia 14 ottobre 2013.

43. European Commission Referral Guidelines for imaging. Rad Protect 2001;118:1-125.

44. Cristofaro M, Busi Rizzi E, Schininà V, et al. Appropriateness: analysis of out patient radiology requests. Radiol Med 2012;117:322-32.

45. The Executive Council of the European Society of Radiology (ESR) and the Council of the World Organization of National Colleges, Academies and Academic Associations of General Practitioners/Family Physicians/ European regional branch (WONCA) Europe. Radiology and primary care in Europe. Insights Imaging 2010;1:46-52.

46. Welch HG, Hayes KJ, Frost C. Repeat testing among medicare beneficiaries. Arch Intern Med 2012;172: 1745-51.

47. Qaseem A, Alguire P, Dallas P, et al. Appropriate use of screening and diagnostic tests to foster high-value, cost conscious care. Ann Intern Med 2012;156:147-9.

48. Sirovich BE, Woloshin S, Schwartz LM. Too little? too much? primary care physicians' views on US health care - a brief report. Arch Intern Med 2011;171:1582-5.

49. Larson DB, Johnson LW, Schnell BM, et al. National trends in CT use in the emergency department: 19952007. Radiology 2011;258:164-73.

50. Schattner A. Test appropriateness index. Am J Med 2012;125:e13.

51. McGregor MJ. Testing 1, 2, 3-Is overtesting undermin- 
ing patient and system health? Can Fam Physician 2012;58:1191-3.

52. Trenti T. Appropriatezza e laboratorio. Presentazione al Seminario "L'appropriatezza diagnostica, terapeutica e organizzativa in area medica: l'impegno dei professionisti delle Aziende Sanitarie della provincia di Reggio Emilia”, Reggio Emilia 14 ottobre 2013.

53. Descovich C, Nardi R, Ligabue A, et al. Inappropriate emergency laboratory test ordering: defensive or peer evidence shared based medicine? Ital J Med 2008;2:13-22.

54. Cook S. Experts' guide to saving money in health. BMJ 2010;340:c1281.

55. Miyakis S, Karamanof G, Liontos M, Mountokalakis TD. Factors contributing to inappropriate ordering of tests in an academic medical department and the effect of an educational feedback strategy. Postgrad Med J 2006;82:823-9.

56. Srivastava R, Bartlett WA, Kennedy IM, et al. Reflex and reflective testing: efficiency and effectiveness of adding on laboratory tests. Ann Clin Biochem 2010; 47:223-7.

57. Harbarth S, Holeckova K, Froidevaux C, et al. Diagnostic value of procalcitonin, interleukin- 6 and interleukin- 8 in critically ill patients admitted with suspected sepsis. Am J Respir Crit Care Med 2001;164: 396-402.

58. Tissot F, Lamoth F, Hauser PM, et al. $\beta$-glucan antigenemia anticipates diagnosis of blood culture-negative intraabdominal candidiasis. Am J Respir Crit Care Med 2013;188/9:1100-9.

59. AIFA - Osservatorio Nazionale sull'impiego dei farmaci. L'uso dei farmaci in Italia - Rapporto Nazionale; gennaio-settembre 2013. Available from: http://www.agenziafarmaco.gov.it/sites/default/files/Rapporto_OsMed_g enset2013.pdf

60. Simon C. Prescribing in the elderly. InnovAiT 2008;1:813-9.

61. The American Geriatrics Society 2012. Beers Criteria Update Expert Panel, American Geriatrics Society Updated Beers Criteria for Potentially Inappropriate Medication Use in Older Adults. J Am Geriatr Soc 2012; 60:616-31.

62. Lau DT, Briesacher BA, Touchette DR, et al. Medicare part D and quality of prescription medication use. Drugs Aging 2011;1:797-807.

63. Diabetes Prevention Program Research Group. Reduction in the incidence of type 2 diabetes with lifestyle intervention or metformin. N Engl J Med 2002;346: 393-403.

64. Lindstrom J, Louheranta A, Mannelin M, et al. Lifestyle intervention and 3-year results on diet and physical activity. Diabetes Care 2003;26:3230-6.

65. Lau DT, Briesacher BA, Touchette DR, et al. Medicare part D and quality of prescription medication use. Drugs Aging 2011;1:797-807.

66. National Institute for Health and Clinical Excellence. Medicines adherence: involving patients in decisions about prescribed medicines and supporting adherence; 3 March 2008. Available from: http://guidance.nice.org.uk/ page.aspx ${ }_{0}=267072$

67. Home R. Compliance, adherence, and concordance: implications for asthma treatment. Chest 2006;130: 65S-72S.

68. Cerreta F, Heichler HG, Rasi G. Drug policy for an aging population - the European Medicines Agency's geriatric medicines strategy. N Engl J Med 2012;367: 1972-4.

69. Pitt B. Generic drugs in cardiology: will they reduce health care costs? J Am Coll Cardiol 2004;44:10-3.

70. Zhang Y, Zhou L, Gellad WF. Potential savings from greater use of $\$ 4$ generic drugs. Arch Intern Med 2011;171:468-9.

71. Marzo A, Porro E, Barassi A. Generic drugs: myths, facts, and limitations. Ital J Med 2012;6:146-52.

72. Nardi R, Masina M, Cioni G, et al. Generic - equivalent drugs use in internal and general medicine patients: distrust, confusion, lack of certainties or knowledge? Ital J Med 2014;8:80-109.

73. Pirmohamed M, James S, Meakin S, et al. Adverse drug reactions as cause of admission to hospital: prospective analysis of 18820 patients. BMJ 2004;3:15-9.

74. Nobili A, Licata G, Salerno F, et al. Polypharmacy, length of hospital stay, and in-hospital mortality among elderly patients in hospital internal medicine wards. The REPOSI Study. Eur J Clin Pharmacol 2011;67:507-19.

75. Onder G, Pedone C, Landi F, et al. Adverse drug reactions as cause of hospital admissions: results from the Italian Group of Pharmacoepidemiology in the Elderly (GIFA). J Am Geriatr Soc 2002;50:1962-8.

76. Garattini S. Are me-too drugs justified? J Nephrol 1997;10:283-94.

77. Garattini S. La responsabilità delle Società Mediche nello sviluppo e utilizzo dei farmaci. Lettura nel Convegno: "Globalizzazione e competitività: una sfida per la ricerca indipendente", Roma, Auditorium Ministero della Salute, 12 marzo 2014.

78. Light DW. Effectiveness and efficiency under competition: the Cochrane test. BMJ 1991;303:1253-4.

79. Bloche MG. Beyond the "R Word"? Medicine's New Frugality. NEJM 2012;366:1951-3.

80. Hunter DJ. The impact of the spending review on health and social care. BMJ 2010;341:6022.

81. Cooper C, Starkey K. Disinvestment in health care: A shared vocabulary, language, and narrative of change are needed. BMJ 2010;340:605.

82. Bentley TGK, Effros RM, Palar K, Keeler EB. Waste in the U.S. Health Care System: a conceptual framework. Milbank Q 2008;86:629-59.

83. Nardi R, Berti F, Greco A, et al. Complexity in hospital internal medicine departments: what are we talking about? Ital J Med 2013;7:142-55.

84. Berwick DM, Hackbarth AD. Eliminating waste in US Health Care. JAMA 2012;307:1513-6.

85. Fineberg HV. A Successful and sustainable health system - how to get there from here. N Engl J Med 2012;366:1020-7.

86. McMahon LF, Chopra V. Health care cost and value: the way forward. JAMA 2012;307:671-2.

87. Brody H. From an ethics of rationing to an ethics of waste avoidance. N Engl J Med 2012;366:1949-51.

88. Kimberton Clinic. Sustainable medicine. Available from: http://kimbertonclinic.com/what.htm

89. Schiff GD, Galanter WL, Duhig J, et al. Principles of conservative prescribing. Arch Intern Med 2011;171: 1433-40.

90. Goede V, Hallek M. Elderly patients in clinical trials: new fitness-adapted concepts. Internist 2007;48:1232-7. 
91. Balducci L, Extermann M. Management of cancer in the older person: a practical approach. Oncologist 2000;5:224-37.

92. Goede V, Hallek M. Optimal pharmacotherapeutic management of chronic lymphocytic leukaemia: considerations in the elderly. Drugs Aging 2011;28:163-76.

93. Shanafelt T. Treatment of older patients with chronic lymphocytic leukemia: key questions and current answers. Hematology 2013:158-67.

94. Coleman EA, Berenson RA. Lost in transition: challenges and opportunities for improving the quality of transitional care. Ann Intern Med 2004;140:533-6.

95. Bettger JP, Alexander KP, Dolor RJ, et al. Transitional care after hospitalization for acute stroke or myocardial infarction: a systematic review. Ann Intern Med 2012;157:407-16.

96. Krumholz HM, Currie PM, Riegel B, et al. A taxonomy for disease management: a scientific statement from the American Heart Association Disease Management Taxonomy Writing Group. Circulation 2006;114:1432-45.

97. Bodenheimer T, Lorig K, Lolman H, et al. Patient self management of chronic diseases in primary care. JAMA 2002;288:2469-75.

98. Casalino LP, Rittenhouse DR, Gillies RR. Shortell SM. Specialist physician practices as patient-centered medical homes. NEJM 2010;362:1555-8.

99. Tappenden P, Campbell F, Rawdin A, et al. The clinical effectiveness and costeffectiveness of home-based, nurse-led health promotion for older people: a systematic review. Health Techn Assess 2012;16:1366-5278.

100. Phillips LS, Branch WT, Cook CB, et al. Clinical inertia. Ann Intern Med 2001;135:825-34.

101. Giugliano D, Esposito K. Clinical inertia as a clinical safeguard. JAMA 2011;305:1591-2.

102. Cabana MD, Rand CS, Powe NR, et al. Why don't physicians follow clinical practice guidelines? A framework for improvement. JAMA 1999;282:1458-65.

103. Allen JD, Curtiss FR, Fairman KA. Nonadherence, clinical inertia, or therapeutic inertia? JMCP 2009;15: 690-5.

104. O'Connor PJ, Sperl-Hillen JM, Johnson PE, et al. Clinical inertia and outpatient medical errors. April 2005. Agency for Healthcare Research and Quality. Available from: http://www.ahrq.gov/downloads/pub/advances/ vol2/OConnor.pdf

105. Thistlethwaite JE, Ajjawi R, Aslani P. The decision to prescribe: influences and choice. InnovAiT 2012; 3:237-43.

106. Nardi R, Fabbri T, Belmonte G, et al. Internal medicine, complexity, evidence based medicine, almost "without evidences". Ital J Med 2009;3:191-200.

107. Alderson P. Absence of evidence is not evidence of absence. BMJ 2004;328:1016-7.

108. Lee EO, Emanuel EJ. Shared decision making to improve care and reduce costs. NEJM 2013;368:6-8.

109. Brett AS, McCullough LB. Addressing requests by patients for nonbeneficial interventions. JAMA 2012;307: 149-50.

110. Campbell EG, Pham-Kanter G, Vogeli C, Iezzoni LI. Physician acquiescence to patient demands for brand name drugs: results of a national survey of physicians.
JAMA Intern Med 2013. [Epub ahead of print]. 111. Fenton JJ, Jeran AF, Bertakis KD, Franks P. The cost of satisfaction. a national study of patient satisfaction, health care utilization, expenditures, and mortality. Arch Intern Med 2012;172:405-11.

112. Paterniti DA, Fancher TL, Cipri CS, et al. Getting to "No". Strategies primary care physicians use to deny patient requests. Arch Intern Med 2010;170:381-8.

113. Kaplan RM, Babad YM. Balancing influence between actors in healthcare decision making. BMC Health Serv Res 2011;11:85.

114. Truog RD. Patients and doctors - the evolution of a relationship. NEJM 2012;366:581-5.

115. Institute of Medicine. Crossing the Quality Chasm: A New Health System for the $21^{\text {st }}$ Century. Washington, DC: National Academy Press; 2001.

116. Ivers N, Jamtvedt G, Flottorp S, et al. Audit and feedback: effects on professional practice and healthcare outcomes. Cochrane Database Syst Rev 2012;6: CD000259.

117. Good Stewardship Working Group. The "Top 5" lists in primary care: meeting the responsibility of professionalism. Arch Intern Med 2011;171:1385-90.

118. Brody H. Medicine's ethical responsibility for health care reform - the Top Five list. NEJM 2010;362:283-5.

119. Volpp KG, Loewenstein G, Asch DA, Choosing wisely. Low-value services, utilization, and patient cost sharing. JAMA 2012;308:1635-6.

120. Choosing Wisely. Lists. Available from: http://www. choosingwisely.org/doctor-patient-lists Accessed: 25 Marzo 2014.

121. Slow Medicine. La medicina sobria, rispettosa, giusta. Available from: http://www.slowmedicine.it Accessed: 25 Marzo 2014.

122. Porter ME, Olmsted Teisberg E. How physicians can change the future of health care. JAMA 2007;297: 1103-111.

123. Kienle GS, Kiene H. Clinical judgement and the medical profession. J Eval Clin Pract 2011;17:621-7.

124. Feinstein AR. Clinical judgement. Baltimore: Williams \& Wilkins; 1967. p 21.

125. Stine NW, Chokshi DA. Opportunity in austerity - a common agenda for medicine and public health. NEJM 2012;366:395-7.

126. Chien A, Rosenthal MB. Waste not, want not: promoting efficient use of health care resources. Ann Intern Med 2013;158:678.

127. Grady D, Redber RF. Less is more. How less health care can result in better health. Arch Intern Med 2010;170:749-50.

128. The JAMA network. Specialties \& Topics, Less is more. Available from: http://jamanetwork.com/collection.aspx? categoryid $=6017$

129. Institute of Medicine. Best care at lower cost: the path to continuously learning health care in America, Recommendations, December 2012. Available from: http://www.iom.edu/bestcare

130. D'Amore. The doctor and the patient: doing too much or too little. Ital J Med 2013;7:135-6.

131. Palfrey S. Daring to practice low-cost medicine in a high-tech era. NEJM 2011;364:e21. 


\section{APPENDICE}

G. Civardi (Firenzuola), G. Uomo (Napoli), M. Ventrucci, M. Masina (Bentivoglio), M. Marvisi (Cremona), A. Sacchetta (Conegliano), F. Salvati (Ortona), G. Ballardini (Rimini), C. Puoti (Marino), M. Costantini (Genova), L. Magnani (Voghera), Giampiero Beltramello (Bassano del Grappa), G. Bajocchi, C. Salvarani (Reggio Emilia). A. Salsi (Bologna), M. Reta (Bologna), P. Leandri (Bologna), G. Di Pasquale (Bologna), C. Descovich (Bologna), A. Santoro (Bologna). 


\title{
Il medico ed il suo paziente: fare giusto, non troppo né troppo poco
}

\author{
Francesco D'Amore \\ Primario f.r. di Medicina Interna, Roma, Italia
}

\section{RIASSUNTO}

Il lavoro del medico si svolge su un crinale stretto tra il fare troppo o troppo poco, un terreno accidentato che il medico deve percorrere con equilibrio e senso di solidarietà, consapevole di essere, nel prossimo futuro, protagonista di un rinnovamento dei rapporti con la società. Noi internisti ospedalieri vogliamo proporre alcune possibili idee per un'iniziativa di livello nazionale al fine di realizzare una Medicina Interna ospedaliera sobria ed efficiente.

Il lavoro del medico si svolge su un crinale stretto tra il fare troppo o troppo poco, un terreno accidentato che il medico deve percorrere con equilibrio e senso di solidarietà, consapevole di essere, nel prossimo futuro, protagonista di un rinnovamento dei rapporti con la società.

Nardi ed altri hanno interpretato l'esigenza, quasi la necessità, di dare, attraverso il loro documento programmatico Toward a sustainable and wise healthcare approach: potential contributions from hospital Internal Medicine Departments to reducing inappropriate medical spending, pubblicato recentemente sull'Italian Journal of Medicine, ${ }^{1}$ un contributo per una visione complessiva dei tanti aspetti che costituiscono l'atto medico, la relazione tra medico e paziente ed il complicato sistema assistenziale che sono diventati così intricati e dispersivi da causare incertezza e confusione.

All'interno di questo lavoro si indicano strumenti per una metodologia clinica saggia ed efficace $N o i$ vorremmo proporre alcune possibili idee per un'iniziativa di livello nazionale al fine di realizzare una Medicina Interna ospedaliera sobria ed efficiente.

Un modello originale di comportamenti, cioè, funzionale e prezioso anche nelle non poche realtà italiane nelle quali esistono le più svariate carenze e nelle quali il medico paga ogni disservizio ed ogni inadeguatezza.

Corrispondente: Francesco D’Amore, via Rodolfo Lanciani 2, 00162 Roma, Italia.

Fax: +39.06.3729885.

E-mail: dottdamore@libero.it

Parole chiave: medicina saggia, sobria, frugale ed efficiente.

Articolo pubblicato secondo la Creative Commons Attribution NonCommercial 3.0 License (CC BY-NC 3.0).

CCopyright F. D'Amore, 2014

Licensee PAGEPress, Italy

QUADERNI - Italian Journal of Medicine 2014; 2:49-51
Per questo è necessario che il medico tenga una condotta chiara, appropriata e supportata dall'evidence-based medicine, coerente con le condizioni cliniche del paziente e con le sue condizioni psicologiche, sociali, familiari, ambientali. In sostanza i rischi estremi da evitare sono, sia un atteggiamento timoroso che comporterebbe la trasformazione della società in una comunità di malati, come venne rappresentata da Jules Romains nel suo Dott. Knock o Il trionfo della medicina, ${ }^{2}$ sia una condotta negligente che provocherebbe altrettanti se non maggiori danni. Il lavoro di Nardi dedica a questo proposito due interessanti capitoli, la sovradiagnosi e l'inerzia professionale con i relativi costi che ne possono derivare.

Per superare questi atteggiamenti, l'internista ha, forse, la responsabilità di aver fatto sentire poco la sua voce sul piano culturale e formativo, sopraffatta, per la verità, dal clamore assordante prodotto dalle cure rivolte al singolo organo. Scrive Daniel Pennac che al medico di oggi importa soltanto il puzzle cellulare, il corpo radiografato, ecografato, tomografato, analizzato, il corpo biologico, genetico, molecolare, la fabbrica di anticorpi. ${ }^{3}$

Questa disintegrazione del corpo in tanti pezzi, conoscenza per 'riduzioni', come la definisce Ivan Cavicchi ${ }^{4}$ ha comportato il fenomeno della consulenza dei più svariati specialisti, amplificato anche e soprattutto dalla spada incombente della denuncia. L'eccessivo ricorso alle consulenze è stato definito da Richard M. Plotzker, endocrinologo statunitense, consultorrhea, un termine esplicitamente ironico per una tendenza difficile da contenere. ${ }^{5}$ Una tendenza che potrebbe trovare un argine nel ragionamento clinico rigoroso come parte integrante delle cure, come scrive lo stesso Plotzker.

Esiste, però, il bisogno, al di là dei tanti e vari consulenti, di uno specialista, come l'internista, che recuperi l'identità e l'integrità della persona, sappia individuare le priorità cliniche e programmi interventi diagnostici e terapeutici coerenti. L'internista è consapevole che il paziente, spesso oppresso dalle incer- 
tezze della non linearità della biologia, non è solo materia ma un insieme di speranze, di sogni, di desideri, di pudori, e per questo deve essere abile a percepire anche le sue difficoltà e, di fronte a questo smarrimento, dovrebbe proporsi come un sostegno attivo e decisivo, tenendo presente, fra l'altro, quanto scrivono Nardi et al.: Non è sempre vero che più è meglio. ${ }^{1} \mathrm{Nel}$ ricordare il famoso report del Journal of the American Medical Association (JAMA), abbiamo, invece, sottolineato il concetto che meno è più, dal quale si è diffuso il principio della migliore cura al prezzo più basso, ${ }^{6}$ condiviso anche dall'Istituto statunitense di Medicina.

Uno degli aspetti in cui il medico deve esprimere preparazione e sensibilità è quello della riconciliazione terapeutica che viene messa alla prova in momenti cruciali come quelli dell'ingresso in ospedale, della dimissione e di ogni controllo ambulatoriale. Sono momenti questi nei quali le cure prescritte si incrociano e si modificano in relazione alle condizioni cliniche, di sicurezza e di compliance del paziente. ${ }^{?}$

Nardi aggiunge e sottolinea che noi abbiamo imparato (o reimparato) la pratica medica in un modo non così dipendente dalla tecnologia. La nostra assistenza sanitaria è stata studiata, infatti, per i bisogni reali della persona. Proprio per questo ricordiamo che la diagnosi è basata, nella maggior parte dei casi, sull'anamnesi e sull'esame obiettivo e che l'ultima medicina assunta non è necessariamente la migliore. ${ }^{1}$

Negli Stati Uniti, l'American Board for Internal Medicine (ABIM) Foundation, ha proposto il modello della choosing wisely, ${ }^{8}$ abbracciato da numerose Società scientifiche per aiutare medici, pazienti e famiglie a selezionare le strade più razionali sia clinicamente che economicamente. In realtà questo modello nulla aggiunge alla medicina non improvvisata, non autoreferenziale, libera da condizionamenti, fondata rigorosamente sul metodo e capace di definire le priorità della complessità. FADOI, con il sistematico studio di Nardi, ha voluto riferirsi a modelli originali di medicina interna ospedaliera basata sulla ricerca di strumenti sensati ed efficienti di metodologia clinica. Una pratica che comporta interventi utili, selettivi, validi, ragionevoli, capaci di selezionare le priorità e di identificare l'aspetto più importante di molti problemi complessi, spesso sovrapponibili a situazioni diverse subspecialistiche.

FADOI ha proposto, quindi, dieci punti per una slow medicine, d'accordo con la società scientifica Slow Medicine e consiglia una rispettosa, sostenibile e ragionevole medicina ospedaliera per pazienti complessi internistici (Tabella 1).

FADOI promuove, in sostanza, una medicina che conserva i tratti basic della metodologia clinica, dal-

Tabella 1. Il decalogo FADOI per una slow medicine: le raccomandazioni generali degli Internisti ospedalieri per una medicina sobria, rispettosa e giusta.

Nel paziente internistico complesso e con co-morbilità multiple esercita una proattiva selezione delle priorità, ordinando i vari problemi rispetto al loro effettivo valore clinico

Limita la richiesta di consulenze specialistiche a quelle effettivamente necessarie rispetto ai risultati attesi

Prima di richiedere accertamenti nuovi, verifica: se sono già stati effettuati in precedenza e quando; quali informazioni aggiuntive essi possono effettivamente dare al medico nelle decisioni per la gestione del paziente e quali rischi si corrono

Informa e coinvolgi il paziente nella cura e nelle scelte che lo riguardano: cerca di personalizzare la gestione della malattia e della terapia, in funzione dei bisogni attivi e reali del paziente, dei suoi valori e delle sue giuste preferenze, nonché dei potenziali rischi. In base alle migliori conoscenze disponibili cerca di esplicitare: quali obiettivi clinici possono essere realisticamente perseguiti e quali possono essere le potenziali richieste improprie da parte del paziente e dei suoi famigliari

Nelle ultime fasi di vita del paziente internistico, astieniti da procedure e/o esami invasivi. Seleziona le cure in base alla necessità di garantire un'adeguata qualità di vita e il trattamento efficace del dolore

Esercita la riconciliazione terapeutica* all'ingresso del paziente in ospedale, alla sua dimissione e ad ogni visita ambulatoriale

Per il trattamento di patologie croniche, in particolare per i nuovi pazienti, usa i farmaci equivalenti, in base alle caratteristiche del paziente, della malattia e dei principi attivi

Pianifica la dimissione fin dall'ingresso del paziente in ospedale, sulla base: della valutazione globale del paziente (clinica, funzionale, psicologico-cognitiva, economico-sociale e famigliare), dell'individuazione dei casi di dimissione difficile e delle risorse disponibili nel territorio per la continuità assistenziale

Alla dimissione dall'ospedale riduci il più possibile il numero dei farmaci prescritti rispetto a quelli già assunti a domicilio prima del ricovero

Alla dimissione dall'ospedale fornisci al paziente, ai famigliari e ai caregivers i riferimenti necessari per l'autogestione della malattia (quando possibile), delle medicine e dei presidi necessari; evidenzia i sintomi e i segni da controllare per prevenire un peggioramento e ricorda i riferimenti su a chi rivolgersi in caso di bisogno, le modalità e gli appuntamenti per il dopo-dimissione, i servizi domiciliari attivati o da attivare

Fonte: http://www.slowmedicine.it/pdf/Decalogo\%20FADOI\%20-\%20Slow\%20Medicine.pdf

* Riconciliazione: è il processo che partendo dal confronto tra la lista dei farmaci assunti dal paziente, come risultanti dalla ricognizione, e quelli che dovrebbero essere somministrati al paziente nella particolare circostanza, permette di giungere alla formulazione di una decisione prescrittiva farmacologica corretta. 
l'analisi degli elementi anamnestici ed obiettivi alla funzione della diagnostica differenziale, e che si avvale dell'uso appropriato di ogni mezzo tecnologico e terapeutico.

I dieci punti rappresentano i profili di una medicina che considera il ruolo attivo del paziente come un fattore fondamentale per raggiungere gli obiettivi concordati con il medico che lo guida sulla strada dell'assistenza e della terapia.

\section{Bibliografia}

1. Nardi R, Berti F, Fabbri L, et al. Toward a sustainable and wise healthcare approach: potential contributions from hospital Internal Medicine Departments to reducing inappropriate medical spending. Ital $\mathrm{J}$ Med 2013;7:65-81.
2. Romains J. Dott. Knock ou Le triomphe de la médicine. Pièce de théâtres. Paris; 1923.

3. Pennac D. Storia di un corpo. Milano: Feltrinelli; 2012.

4. Cavicchi I. Ripensare la medicina. Torino: Bollati Boringhieri; 2004.

5. Plotzker RM. Where's endocrinology in "choosing wisely"? Excesses in diabetes and endocrinology. Available from: http://www.medscape.com/viewarticle/782406

6. The JAMA network. Specialties \& topics, less is more. Available from: http://jamanetwork.com/collection. Aspx?CategoryID-6017

7. Kwan JL, Lo L, Sampson M, Shojania KG. Medication reconciliation during transitions of care as a patient safety strategy: a systematic review. Ann Intern Med 2013;158:397-403.

8. Volpp KG, Loewenstein G, Asch DA. Choosing wisely. Low-value services, utilization, and patient cost sharing. JAMA 2012;308:1635-6. 


\title{
Il decalogo ANìMO per un'assistenza Slow Medicine: le raccomandazioni generali degli infermieri di Medicina Interna per un'assistenza sobria, rispettosa e giusta
}

\author{
Gabriella Bordin, ${ }^{1}$ Moira Bonfanti, ${ }^{2}$ Francesca Calabria, ${ }^{3}$ Rina Cossu, ${ }^{4}$ Caterina Cozzi,${ }^{5}$ Tonino Esposito,${ }^{6}$ \\ Claudia Ganzini, ${ }^{7}$ Elena Gragnoli, ${ }^{8}$ Rosalba Leotta, ${ }^{9}$ Mariapaola Lince, ${ }^{10}$ Lucia Lucci, ${ }^{11}$ Giacinta Manfroni, ${ }^{12}$ \\ Marilisa Martini, ${ }^{13}$ Giovanna Pentella, ${ }^{14}$ Nicoletta Postal,,${ }^{15}$ Roberta Rapetti, ${ }^{16}$ Ornella Teti, ${ }^{17}$ Giuditta Zocchi, ${ }^{18}$ \\ Annunziata Zuccone, ${ }^{19}$ Claudia Gatta ${ }^{20}$
}

${ }^{1} \mathrm{CPSE}$ - ULSS 8 Asolo (TV); ${ }^{2} \mathrm{CPSE}$ - Us1 11 Empoli; ${ }^{3} \mathrm{CPSE}$ - Ospedale Buccheri La Ferla, Palermo; ${ }^{4} \mathrm{CPSE}$ - Ospedale di Tempio Pausania (OT); ${ }^{5} \mathrm{CPSI}$ - Ospedale Mazzini, Teramo; ${ }^{6} \mathrm{CPSE}$ - Ospedale F. Veneziale, Isernia; ${ }^{7} \mathrm{CPSE}$ - Ospedale Latisana/bassa friulana, Udine; ${ }^{8} \mathrm{CPSI}$ - Ospedale Città di Castello (PG); ${ }^{9} \mathrm{CPSE}$ - Ospedale S. Giovanni di Dio, Crotone; ${ }^{10} \mathrm{CPSE}$ - AO Santa Maria Nuova, Reggio Emilia; ${ }^{11} \mathrm{CPSE}$ - Ospedale V. Fazzi, Lecce; ${ }^{12} \mathrm{CPSE}$ - PO Civitanova Marche - Area vasta 3 Marche; ${ }^{13}$ Referente rapporti con le regioni - Anìmo; ${ }^{14} \mathrm{CPSE}$ - Ospedale Fatebenefratelli, Napoli; ${ }^{15} \mathrm{CPSE}$ - APSS Trento; ${ }^{16} \mathrm{CPSE}$ - Ospedale San Paolo, Savona; ${ }^{17} \mathrm{CPSE}$ - AO di Legnano (MI); ${ }^{18} \mathrm{CPSE}$ - Ospedale Sandro Pertini, ASL Roma $\mathrm{B} ;{ }^{19} \mathrm{CPSE}$ - Ospedale San Donato, USL 8 Arezzo; ${ }^{20} \mathrm{CPSE}$ - ASL BI, Biella, Italia

\section{Introduzione}

L'Associazione di Infermieri di Medicina Interna (ANİMO) è stata coinvolta, con altre associazioni infermieristiche, dalla Federazione dei collegi degli infermieri italiani (IPASVI) a partecipare alle iniziative di Slow Medicine.

Molto interesse ha manifestato la partecipazione a questo progetto che incarna pienamente il codice deontologico $^{1}$ e il profilo dell'infermiere. ${ }^{2}$ Un interesse che nasce non solo dalla condivisione della necessità di contrastare l'inappropriatezza (di azioni e prescrizioni), dalla quale derivano svantaggi sia in termini di aumento dei rischi per la persona assistita sia in termini di spreco di risorse, ma anche dall'opportunità di confronto che si è creata allargando questo progetto alle altre professioni sanitarie.

Per noi infermieri si apre infatti l'opportunità per costruire, attraverso un'analisi del nostro esercizio professionale, un'alleanza tra i professionisti della salute (e non solo della malattia) con i cittadini.

Alleanze che hanno l'obiettivo di sostenere e facilitare scelte consapevoli.

Corrispondente: Gabriella Bordin, CPSE, ULSS 8 Asolo (TV) E-mail: g.bordin@libero.it

Articolo pubblicato secondo la Creative Commons Attribution NonCommercial 3.0 License (CC BY-NC 3.0).

(C) Copyright G. Bordin et al., 2014

Licensee PAGEPress, Italy

QUADERNI - Italian Journal of Medicine 2014; 2:52-53
Come infermieri se l'inappropriatezza prescrittiva sembra riguardarci relativamente poco, con uno sguardo più attento si può valutare l'inadeguatezza delle.... rispetto a .... quella persona specifica. Quale essenza base del ragionamento, abbiamo la responsabilità di evidenziare e considerare nell'azione l'alterità e la specificità della persona che si sta assistendo, le sue esigenze all'interno di un progetto di vita che si sta magari avvicinando al termine: è forse l'unico modo per evitare di fare cose senza senso, vedi ad esempio la programmazione di piani di mobilizzazione nei pazienti in fase terminale.

Ricercare il senso delle cose richiama ad un'azione che ha la radice sicuramente incardinata nel terreno scientifico ma ha, quale direttiva, la ricerca del senso, del significato per la persona, per le persone che ho di fronte.

A tale proposito diventa quindi fondamentale ragionare in un'ottica interdisciplinare ed aprire un dialogo non solo con le altre associazioni infermieristiche ma con le società scientifiche mediche e con i professionisti con i quali ci si trova quotidianamente a lavorare, con obiettivi comuni, per lo stesso paziente.

Altra importante riflessione è nata dalla domanda: cosa significa essere un infermiere slow e cosa significa erogare un'assistenza sobria rispettosa e giusta?

ANIMO ha cercato di rispondere al quesito con un decalogo, sulla scia di quanto già proposto da $\mathrm{FADOI},{ }^{3}$ a dimostrazione della condivisione dei valori di fondo e delle linee di indirizzo dell'agire quotidiano che accomuna le due società scientifiche, un documento che invita il singolo professionista a fermarsi e a pensare all'obiettivo principale della nostra disciplina che non è la guarigione dalla malattia ma la ripresa della massima autonomia del soggetto e la capacità e di gestire 
il proprio stato di salute, alle strategie che dobbiamo mettere in campo dalle pratiche evidence based alla comunicazione, dall'educazione alla personalizzazione degli interventi, ${ }^{4}$ ai momenti cruciali del percorso del paziente ricoverato in medicina: l'accoglienza, la gestione della terapia farmacologica ${ }^{5}$ la dimissione, ${ }^{6} \mathrm{e}$ a tutto quello che può essere fatto dall'infermiere, di sobrio rispettoso e giusto nelle ultime fasi di vita della persona. ${ }^{7,8}$

\section{I dieci punti}

i) Identifica, al momento dell'accoglienza in ospedale, le conoscenze del paziente e dei familiari sullo stato di salute e sulle aspettative degli stessi circa gli obiettivi di cura, identificando potenziali richieste improprie.

ii) Informa e coinvolgi il paziente nella cura e nelle scelte che lo riguardano, garantisci tempi, spazi e modi adeguati di comunicazione con il paziente e i familiari, discutendo quali obiettivi assistenziali possono essere realisticamente raggiunti.

iii) Garantisci all'interno dell'equipe un'adeguata comunicazione al fine di veicolare tutte le informazione che riguardano il paziente e il progetto di cura a tutti i componenti del team.

iv) Personalizza gli interventi assistenziali in funzione dei reali bisogni paziente, dei suoi valori e preferenze, nonché dei potenziali rischi.

v) Considera prioritaria, nella pianificazione degli interventi assistenziali, la ripresa dell'autonomia della persona nella gestione del proprio stato di salute.

vi) Attua interventi basati sulle migliori conoscenze disponibili, esplicita quali obiettivi possono essere realisticamente perseguiti.

vii) Collabora con il medico nella ricognizione della terapia e nell'identificazione dei problemi legati alla gestione della stessa evidenziando i fattori: quantità di prescrizioni, storia di mancata aderenza, difficoltà di assunzione dei farmaci, presenza di eventuali rischi concomitanti (cadute, confusione, delirium, ecc.), carenza di supporto.

viii) Pianifica la dimissione fin dall'ingresso del paziente in ospedale $\mathrm{e}$, in base alla valutazione globale (clinica, funzionale, economico-sociale e familiare), individua i casi di dimissione difficile. Identifica e attiva le risorse interne alla famiglia e quelle disponibili nel territorio per la continuità assistenziale.

ix) Identifica, durante il ricovero i bisogni educativi del paziente, dei familiari e dei caregivers circa la gestione dello stato di salute, attua interventi educativi mirati alla gestione della terapia farmacologica e della dieta, all'uso di presidi, al riconoscimento precoce delle complicanze, fornisci indicazioni su quale comportamento adottare e a chi rivolgersi in caso di bisogno, le modalità e gli appuntamenti per il dopo-dimissione, i servizi domiciliari attivati o da attivare.

x) Nelle ultime fasi di vita del paziente astieniti da procedure assistenziali invasive, seleziona gli interventi da attuare a garanzia della dignità della persona, della sua qualità di vita e della gestione dei sintomi disturbanti, in modo particolare il controllo efficace del dolore.

\section{Bibliografia}

1. Federazione Nazionale IPASVI. Codice deontologico dell'infermiere; 2009.

2. Ministero della Sanità. Decreto ministeriale 14 settembre 1994, n. 739. Regolamento concernente l'individuazione della figura e del relativo profilo professionale dell'infermiere. G.U. n. 6, 9/01/1995. Disponibile su: http://www.ipasvi.it/archivio_news/leggi/179/DM14099 4n739.pdf

3. D'Amore F. The doctor and the patient: doing too much or too little. Ital J Med 2013;7:e22.

4. D'Ivernois JF, Gagnayre R. Educare il paziente un approccio pedagogico. Milano: McGraw Hill; 2009.

5. Agenzia Sanitaria e Sociale Regionale, Area Accreditamento e Qualità, Regione Emilia Romagna. Sicurezza nella terapia farmacologica "Processo di ricognizione e di riconciliazione farmacologica per una prescrizione corretta e sicura"; 2011. Disponibile su: http://www.saluter.it/documentazione/rapporti/sicurezza_farmacologica_2.pdf Ultimo accesso: 21/03/2014.

6. Shepperd S, McClaran J, Phillips CO, et al. Discharge planning from hospital to home. Cochrane Database Syst Rev 2010;(1):CD000313.

7. Costantini M, Borreani C, Grubich S. Migliorare la qualità delle cure di fine vita. Un cambiamento possibile e necessario. Trento: Erickson; 2009.

8. Registered Nurses'Association of Ontario (RNAO). End of life during the last days and hours; 2011. Disponibile su: http://rnao.ca/bpg/guidelines/endoflife-care-duringlast-days-and-hours Ultimo accesso: 1/03/2014. 


\title{
Come organizzare l'Area Medica per affrontare le nuove sfide della complessità clinica ed assistenziale
}

\author{
Ilario Stefani, Antonino Mazzone \\ Dipartimento Area Medica, AO Legnano (MI), Italia
}

\section{RIASSUNTO}

Nel contesto dell'attuale riorganizzazione ospedaliera secondo il modello di aggregazione dipartimentale è necessaria la ricerca di nuove forme organizzative, quali l'ospedale basato sull' intensità di cure, UO di tipo dipartimentale, gestione integrata ospedale-territorio per pazienti affetti da patologie croniche. In tale ambito la Medicina Interna può esercitare un ruolo determinante facendosi carico del coordinamento delle cure multiprofessionali.

\section{L'evoluzione epistemologica della medicina}

La Medicina contemporanea si contraddistingue per un'episteme caratterizzata dalla scoperta della complessità, promossa dal riconoscimento del limite insito nei pur straordinari risultati ottenuti dalla tecnologia. ${ }^{1}$

È, questa, una scoperta che proviene dalla filosofia e dalla fisica e che ha prodotto la consapevolezza che il mondo, nel suo insieme, non è lineare, bensì irregolare, variabile, instabile e complesso.

Nel pensiero scientifico contemporaneo, da alcuni anni, è in atto un cambiamento di paradigma della conoscenza. ${ }^{2}$ La linea che guida questo cambiamento trova nella Teoria della Complessità di Morin e Prigogine $^{3}$ la fine del razionalismo classico e l'inizio di una nuova concezione, dove il soggetto è posto al centro dell'osservazione e ne è così permessa un'analisi a tutto tondo. Morin, infatti, sostituisce al concetto di oggetto quello di sistema, non studiando più, o non solo, oggetti-soggetti singoli ma sistemi complessi, organizzati in modo tale secondo una struttura nella quale gli elementi in interazione tra di loro costituiscono un'entità globale: un sistema che è diverso dalla

Corrispondente: Antonino Mazzone, Dipartimento Area Medica, AO di Legnano, via Papa Giovanni Paolo II 1, 20025 Legnano (MI), Italia.

E-mail: medicina2legnano@ao-legnano.it

Parole chiave: organizzazione dipartimentale ospedaliera, intensità di cure, coordinamento multi-professionale, gestione integrata ospedale-territorio.

Articolo pubblicato secondo la Creative Commons Attribution NonCommercial 3.0 License (CC BY-NC 3.0).

(C) Copyright I. Stefani, A. Mazzone, 2014

Licensee PAGEPress, Italy

QUADERNI - Italian Journal of Medicine 2014; 2:54-59 semplice sommatoria delle parti che lo compongono, in un processo di adattamento agli eventi esterni.

Gli scopi della Medicina hanno quindi subito, in questi anni, una dilatazione, frammentandosi e articolandosi in un'ampia serie di obiettivi in rapporto alla conoscenza della malattia, alla terapia e alla relazione terapeutica con il paziente, ma anche alla conoscenza gestionale ed economica dell'attività clinica, all'utilizzo coerente e razionale delle risorse messe a disposizione dalle politiche sanitarie adottate.

La medicina moderna si trova oggi ad affrontare l'esigenza di garantire continuità nella cura e nella conoscenza dell'ammalato e, contemporaneamente, quella di operare in contesti ad alto contenuto tecnologico e ad alta specializzazione.

L'organizzazione può essere definita come un sistema che elabora informazioni, risolve problemi, si esprime con la capacità di trasformare le informazioni che provengono dai suoi componenti e dai suoi clienti per prendere decisioni e dare risposte e risultati appropriati al contesto. ${ }^{4}$ Questo elemento è particolarmente importante in sanità, dove abbiamo assistito negli ultimi decenni a una profonda modificazione del concetto di salute, dell'attesa di salute e di benessere da parte del pubblico.

In tale contesto la Medicina Interna svolge un ruolo fondamentale. La visione allargata delle problematiche cliniche e gli strumenti metodologici che le sono propri consentono alla Medicina Interna di poter affrontare, tanto sul piano diagnostico quanto a livello terapeutico, le sfide della medicina moderna, con un approccio olistico e multidisciplinare e una visione a tutto campo. ${ }^{5}$

Le Società Scientifiche Mediche hanno indicato nell'ambito della Medicina Interna quello spazio concettuale e operativo relativo alle conoscenze sulle malattie di organi e apparati, suscettibili di interagire fra loro e di alterare direttamente i parametri fisiologici dell'organismo; inoltre, l'opera della Medicina Interna è realizzata dal Clinico Medico, dall'Internista che sa avvalersi di una 
metodologia che gli consente una valutazione globale delle problematiche che il malato gli pone.

Questi concetti stanno determinando profonde conseguenze nell'ambito delle discipline mediche, introducendo metodologie di lavoro improntate alla multidisciplinarietà, all'integrazione, all'implementazione, al lavoro contestualizzato. ${ }^{6,7}$

In quest'area vasta, la complessità reca il suo contributo metodologico alla sanità in generale e alla gestione dell'assistenza, così come al contesto clinico e alla stessa formazione medica. La complessa natura della sanità odierna deve adeguarsi alla crescente articolazione della domanda, così come alla differenziazione dei quadri nosografici sempre più inaspettati, e su queste basi realizzare risposte flessibili ai modelli e alle opportunità emergenti.

La complessità nella gestione dell'assistenza può quindi essere sostenuta dal Clinico Medico, dal Medico Internista, capace, all'interno di un sistema adattativo complesso, di svolgere un'azione di coordinamento, d'integrazione, di cooperazione fra le diverse parti che agiscono per ambiti specifici di specializzazione settoriale.

\section{Il cambiamento epidemiologico}

L'invecchiamento della popolazione ed il conseguente incremento dell'età media ha notevolmente aumentato il numero di soggetti affetti da patologie croniche, da patologie multiple e da patologie complesse. Parallelamente le mutate condizioni socioeconomiche del nostro paese e della nostra regione hanno incrementato il numero di soggetti anziani e di soggetti socialmente in difficoltà, privi di una rete familiare in grado di accudirli e garantire loro un supporto adeguato alla gestione della vecchiaia e delle patologie croniche spesso presenti. Si è progressivamente assistito ad un incremento dei soggetti fragili nei quali la cura di un episodio patologico deve necessariamente essere correlata alla cura globale della persona, prevedendo meccanismi di interazione con il territorio e le strutture socio-assistenziali attraverso percorsi di dimissione protetta.

\section{La dipartimentalizzazione}

È in questo ambito concettuale ed epidemiologico che, a partire dall'ultimo decennio, il Sistema Sanitario Italiano è stato oggetto di un profondo ammodernamento volto a promuovere l'efficienza produttiva, l'efficacia e l'appropriatezza dei servizi sanitari attraverso l'introduzione di nuovo assetti organizzativi delle Unità Operative promuovendo l'introduzione di modelli di tipo dipartimentale, promossi in particolare dal D.lgs 502/92, oltre che da normative a livello re- gionale che completano il quadro istituzionale del nuovo modello divisionale.

Diversi autori hanno analizzato gli obiettivi perseguibili attraverso l'implementazione del dipartimento nell'ambito delle strutture sanitarie; in particolare Cicchetti $^{8}$ ne individua quattro: i) organizzativo; ii) clinico; iii) economico; iv) strategico. L'obiettivo organizzativo permette di conseguire un migliore coordinamento delle attività di assistenza necessarie al trattamento di pazienti per cui risulta indispensabile la concorrenza di un sistema complesso di competenze e risorse. L'obiettivo clinico evidenzia la capacità del dipartimento di diffondere nuove conoscenze attraverso l'utilizzo di percorsi diagnostico terapeutici condivisi quale strumento di riduzione della variabilità nelle procedure e nei risultati. L'obiettivo economico concerne il coordinamento di tutte le risorse impiegate all'interno delle aziende sanitarie con la finalità di perseguire efficacia attraverso una migliore gestione delle risorse. Infine l'obiettivo strategico riguarda il miglioramento delle conoscenze utili allo sviluppo futuro delle organizzazioni. Più recentemente gli studi si sono concentrati sulla possibilità di realizzare il governo clinico attraverso il dipartimento, considerato il contesto organizzativo più adeguato per l'implementazione dello stesso. La clinical governance si sviluppa come prospettiva unificante degli aspetti clinici ed economici nelle prestazioni sanitarie, strumento attraverso il quale le organizzazioni del sistema sanitario nazionale possono migliorare continuamente la qualità delle cure, focalizzandosi sull'eccellenza clinica derivante dall'adozione di elevati standard di cura. La clinical governance si snoda in differenti aspetti quali la gestione del rischio clinico, l'adozione di linee guida di dipartimento e di percorsi assistenziali di dipartimento sulla base della evidence based medicine, l'attività di valutazione degli outcome clinici.

\section{Le modalità organizzative nella Unità Operativa di Medicina Interna}

Da queste premesse e dalla riorganizzazione ospedaliera attuata tramite la progressiva realizzazione del modello di aggregazione dipartimentale, è nata la ricerca di nuove forme organizzative quali il modello basato sull' intensità di cure, sia di UO che di tipo dipartimentale, sia i modelli di gestione integrata ospedale-territorio, focalizzati alla gestione dei soggetti affetti da patologie croniche

In questo contesto la Medicina Interna gestisce la diagnosi e la terapia dei pazienti complessi, polipatologici, fragili; offre una risposta efficace alle necessità di assistenza che emerge dai problemi clinici presenti in un territorio, permettendo un utilizzo appropriato delle funzioni specialistiche ad alta tecnologia e la loro concentrazione territoriale; ha la capacità di rispon- 
dere, con il modulo organizzativo più adatto, ai diversi momenti della storia clinica del paziente, dal primo contatto con la struttura ospedaliera (ambulatori, macroattività ambulatoriale complessa) alle fasi più critiche del processo di assistenza (reparti per acuti), fino alla gestione di aree per post-acuti, facendosi carico del coordinamento delle cure multiprofessionali.

\section{Tipologia dei pazienti ricoverati, complessità ed intensità}

In relazione alle caratteristiche di ogni singolo paziente afferente all'ambito della Medicina Interna considerato nella sua interezza la UO Internistica, in una logica di appropriatezza, di promozione della qualità e di efficacia dell' assistenza, dovrebbe essere in grado di offrire uno specifico percorso assistenziale, del quale il tradizionale ricovero ospedaliero costituisce solamente una delle possibilità od una parte di esso.,10

In questo contesto è opportuno introdurre un'ulteriore dimensione della complessità, e cioè il concetto di intensità, strettamente legato al tipo di assistenza di cui il paziente ha bisogno. Nella pratica medica è necessario definire in modo preciso e condivisibile la complessità e il modo in cui affrontarla, poiché questo ha risvolti fondamentali nell'impostazione di strategie diagnostico-terapeutiche e nell'organizzazione dei percorsi sanitari. Questo è il motivo per cui individuare una definizione operativa valida della complessità non ha un significato linguistico, ma garantisce l'efficacia nella comunicazione tra professionisti. ${ }^{9,10}$

Il concetto di intensità è strettamente legato al tipo di assistenza di cui il paziente ha bisogno. Tuttavia, la sua complessità rimarrà la stessa. A seconda della condizione clinica varieranno il grado di severità e intensità di cure necessarie, le figure professionali coinvolte e la loro densità in rapporto ai pazienti. Il corretto utilizzo dei termini complesso, complicato e intenso-severo consentirà anche l'identificazione del percorso assistenziale più appropriato per il paziente, e quindi un utilizzo consono delle risorse. In altri termini, la complessità è trasversale a tutte le intensità, mentre è il grado di severità-intensità che determina la collocazione del paziente appunto per intensità di cura (bassa, media, elevata). Complessità quindi come comorbilità, multimorbilità, o condizioni a genesi fisiopatologica, sempre interconnessa con le variabili di acuzie e cronicità e con il livello di intensità di cura necessario. La visione è quindi multidimensionale $\mathrm{e}$ rigorosamente attenta alle interazioni fra i diversi sistemi e apparati e alla compromissione della salute della persona come emergenza del sistema complesso e non come somma delle sue componenti.

L'obiettivo di analisi efficace ed efficiente del paziente complesso non può prescindere da una riorganizzazione della realtà sanitaria. Questa, essendo ancora incentrata sul ricovero ospedaliero, volto alla cura della singola malattia acuta, e su indicatori di costo basati sul sistema dei diagnosis-related group, non sembra in grado al momento di fornire gli strumenti per monitorare, oltre all'impatto clinico della complessità, quello economico, soprattutto in un momento in cui le risorse erogabili in campo sanitario sono limitate. I costi richiesti per la gestione di un malato complesso sono superiori alla somma delle spese per singola patologia. Inoltre, dal punto di vista della programmazione sanitaria, un'inadeguata valutazione della complessità e il mancato coordinamento tra le varie specialità possono portare a ritardi diagnostico-terapeutici, riammissioni improprie in ospedale, frammentazioni e duplicazioni terapeutiche con allocazione delle risorse inappropriata.

\section{Modello organizzativo per intensità di cura}

L'evoluzione attuale della Medicina persegue modelli di tipo dinamico focalizzati sul paziente, in relazione alla sua severità e criticità e non semplicemente per singola patologia, considerano il paziente nella sua interezza e nella sua polipatologicità, prevedendo approcci integrati e multidisciplinari.

I pazienti ricoverati in urgenza presso una UO di Medicina Interna possono presentare condizioni cliniche differenti e pertanto necessitare di una tipologia diverse di assistenza di tipo medico e infermieristico. Per rispondere a questa variabilità di presentazione si sono affermati modelli organizzativo-assistenziali che tendono a superare il modello statico di Divisione ospedaliera, suddiviso per specializzazione e caratterizzato da un'omogenea distribuzione di pazienti, indipendentemente dalla loro situazione patologica, su tutto il reparto, strutturato in rigidi modelli assistenziali basati su numero di letti piuttosto che sulle caratteristiche dei pazienti presenti.

Il modello assistenziale basato sull' intensità delle cure può essere costruito come modello organizzativo di UO (specie per le realtà più decentrate ove all'interno delle UUOO di Medicina Interna trovano posto settori specialistici e mancano spesso aree intensive specialistiche dedicate) o come modello organizzativo dipartimentale o interdipartimentale (specie negli ospedali di grandi dimensioni dove possono essere strutturate aree di degenza omogenea cui afferiscono pazienti di più unità operative che vi vengono allocati e distribuiti a seconda delle necessità assistenziali).

In questo modello organizzativo si possono identificare differenti livelli assistenziali.

\section{Area di degenza ad alta intensità di cura}

Fase di malattia severa in condizione di non stabilità clinica in pazienti per cui vi sia la necessità di un'osservazione continua e di un approccio terapeu- 
tico personalizzato che per le sue caratteristiche specifiche (età del paziente, comorbilità, ecc.) non necessiti di un accesso ad una unità di terapia intensiva rianimatoria, semintensiva o specialistica.

E' dedicata a pazienti affetti da patologie acute gravi caratterizzate da alterazione di una o più delle funzioni vitali che necessitano di un monitoraggio frequente di tali parametri e di terapie complesse. Coinvolge pazienti che, per età, per comorbilità, per caratteristiche generali, per problemi logistici non sono eleggibili per ricovero presso unità di terapia intensiva o subintensiva di tipo specialistico.

La gestione di tale area prevede una modalità di lavoro di tipo multidisciplinare. Per la gestione di tale area devono essere definite in maniera adeguata le responsabilità di tipo medico e le responsabilità di tipo infermieristico. Devono essere definiti in maniera adeguata protocolli e percorsi di accesso, per il coinvolgimento di specialisti di altre unità operative, e processi e percorsi di dimissione o di trasferimento presso le altre aree di degenza o presso altre unità operative. In queste aree è di particolare rilievo il ruolo del personale infermieristico, specificamente addestrato all'utilizzo dei sistemi di monitoraggio così come all'utilizzo dei principali presidi terapeutici secondo protocolli condivisi e predefiniti.

\section{Area di degenza per pazienti acuti}

Fase di malattia ancora in acuzie, ma con minor grado di instabilità.

Dedicata a pazienti affetti da patologie acute potenzialmente a rischio di evoluzione negativa se non trattati tempestivamente ed in maniera adeguata. $\mathrm{Si}$ tratta di pazienti che presentano una condizione di relativa stabilità clinica, spesso anziani nei quali la coesistenza di più patologie croniche con l'acuzie motivo del ricovero pone indicazione a ricovero ospedaliero. Pur necessitando di adeguata sorveglianza e controllo evolutivo, non presentano indicazioni ad un monitoraggio in continuo delle principali funzioni vitali.

\section{Area di degenza a bassa intensità di cura}

Paziente stabilizzato, ma ancora a potenziale elevato rischio di ricadute o complicanze con necessità di terapia ospedaliera complessa.

I dati della letteratura indicano che la popolazione in ricovero ordinario in Medicina comprende una quota del $20 \%$ circa di soggetti gestibili in un'area a caratteristiche differenziate di assistenza, in quanto non necessitano più di monitoraggio clinico intensivo, pur richiedendo controlli medici ravvicinati e sostegno infermieristico per garantire le funzioni d'organo, l'espletamento delle attività quotidiane $\mathrm{e}$ la riattivazione funzionale.

Si tratta di una popolazione ben definita, costituita prevalentemente da grandi anziani che, dopo l'evento acuto, presentano difficoltà di stabilizzazione e ripresa e quindi richiedono maggior tempo di ricovero a completamento della degenza per ultimare i controlli clinici e strumentali necessari ed eventualmente per calibrare nella maniera più idonea l'assetto terapeutico da suggerire al domicilio. In queste aree, ove i pazienti sono caratterizzati da una ormai raggiunta buona stabilità clinica e dalla necessità di una sorveglianza e di un monitoraggio intensivamente molto minori, potranno trovare temporaneamente posto anche quei pazienti per i quali, per motivi sanitari o socio-assistenziali o per la combinazione di entrambi, è stata posta indicazione alla dimissione protetta.

\section{La continuità assistenziale}

L'approccio di sistema, una volta raggiunta la comprensione del malato complesso, deve proporsi anche di elaborare una proposta di riorganizzazione e ripianificazione dell'assistenza sanitaria in fase di dimissione dal setting per acuti, che sia in grado di gestire quadri clinici di elevata complessità. Diviene, infatti, prioritario, una volta identificati i determinanti della complessità, proporre nuove strategie di ricerca che includano $i$ quadri di alta complessità clinica. È necessario definire e attuare percorsi diagnostici e assistenziali che possano permettere una gestione coordinata, multiprofessionale e a lungo termine del paziente complesso tali da garantire una continuità assistenziale ospedale-territorio, in modo che il territorio possa farsi carico dell'accoglienza del paziente dopo un'ospedalizzazione e delle strategie di prevenzione secondaria.

Nasce quindi l'esigenza di rivisitare criticamente i percorsi assistenziali e di sperimentare nuove formule organizzative dell'assistenza basate sulla gestione integrata, costruita su percorsi assistenziali condivisi. La potenzialità fondamentale dell'approccio di disease management è di far convergere le energie dei vari attori del sistema su obiettivi comuni, seppur con responsabilità diverse, evitando in tal modo una progettualità non concordata $\mathrm{e}$ frammentaria, spesso incapace di incidere significativamente sui risultati complessivi del sistema assistenziale.

La nuova cultura sistemica vede il Distretto e l'Ospedale come componenti complesse di un continuo indissolubile sul tema dell'appropriatezza in risposta ai fabbisogni assistenziali. Il concetto di appropriatezza, infatti, non va applicato soltanto alle attività clinico-assistenziali ma anche al luogo (setting). L'appropriatezza del luogo di cura e del professionista più pertinente (non solo in termini di competenze ma anche di tecnologie e di tempi disponibili) per i fabbisogni del paziente è un prerequisito di economicità $\mathrm{e}$ di qualità complessiva sia dell'offerta sia della relazione servizio-utente. 
La gestione del paziente in dimissione è essenziale per garantire un'appropriata prosecuzione dell'assistenza sul territorio. L'organizzazione di questa fase del processo assistenziale richiede l'identificazione del bisogno di cura della persona alla dimissione, la complessità dei suoi bisogni socio-assistenziali e verifica $\mathrm{i}$ requisiti per il suo soddisfacimento. La modalità operativa prevede l'elaborazione di percorsi di dimissione protetta che contemplino il bisogno di cura e le problematiche collegate a nuove disabilità o a situazioni logistico familiari (persona che vive sola, assenza di caregiver). Esistono inoltre situazioni cliniche che necessitano di una prosecuzione di monitoraggio (patologie ad andamento cronico quali broncopneumopatia cronica ostruttiva, scompenso cardiaco, ecc.) con percorsi clinico assistenziali integrati ospedale-territorio, coinvolgendo il medico di medicina generale. In questo ambito le UUOO di Medicina Interna, in collaborazione con i servizi territoriali ed i dipartimenti di cure primarie sono chiamate a svolgere un ruolo fondamentale condividendo appositi percorsi atti a gestire e trattare l'ambito della cronicità.

\section{Proposte}

L'organizzazione ospedaliera in unità operative, favorita dalla crescita delle competenze specialistiche, fortemente legate allo sviluppo tecnologico degli anni '70-'80, ha introdotto rilevanti elementi di frammentazione nel processo di cura, producendo un approccio parziale al paziente, con ovvie conseguenze sul piano dell'efficacia dell'intervento e dei costi sostenuti. Nell'attuale visione sistemica diviene necessario recuperare spazi di integrazione e coordinamento, affinchè, nell'ambito dei percorsi di cura, si affermi una continuità assistenziale di tipo multidisciplinare che, allo stesso tempo, preservi le varie linee di specializzazione.

La scelta del modello dipartimentale sembra rispondere meglio a tali esigenze, ri-progettando l'assetto organizzativo ospedaliero tramite l'accorpamento di unità operative strettamente correlate sul piano clinico. Nella dimensione di area di degenza dipartimentale, considerando un maggior numero sia di posti letto sia di specialisti interessati, servono modifiche organizzative correlate al grado di complessità, sostenendo la condivisione di risorse umane, fisiche e di know-how secondo la prospettiva della clinical governance.

\section{Il Dipartimento di Area Medica}

Che la Medicina interna debba rappresentare il nucleo fondamentale di tale modello organizzativo lo dimostra, oltre alla vision delle problematiche cliniche e gli strumenti metodologici che le sono propri, la sua estensione capillare su tutto il territorio nazionale, che permette alla sue UUOO di dare risposta ad oltre i tre quarti dei ricoveri d'urgenza, la cui grande maggioranza riguarda patologie acute o croniche riacutizzate, con pazienti altamente complessi e bisognosi di cure che prevedono competenze di tipo multiprofessionale.

La scelta delle unità operative da accorpare in un dipartimento deve tenere conto sia delle affinità culturali delle discipline afferenti, sia delle modalità gestionali delle patologie che vi sono trattate.

La mission del dipartimento di Medicina sarà quella di trattare patologie di tipo acuto, organizzando l'assistenza attorno all'idea della cura secondo il modello dell'intensità. I nuovi modelli organizzativi rendono necessaria l'introduzione di figure nuove, la principale delle quali è il responsabile del caso (tutor), ruolo di riferimento sia per il personale che lavora nell'area sia per il paziente che, altrimenti, proverebbe la sensazione di avere perso un essenziale punto di riferimento nell'esperienza del ricovero e del personale percorso di cura. Nei confronti del paziente diviene pertanto necessario un grande sforzo di comunicazione.

In ragione della specificità del medico Internista nel gestire la complessità appare verosimile che la maggior parte della casistica sia gestita da tale figura; se nel dipartimento sono presenti altre specialità di area i medici di relativa appartenenza gestiranno direttamente $\mathrm{i}$ casi che richiedono particolari competenze, fornendo il loro prezioso e costante contributo come consulenti (consultant) per tutte le altre situazioni.

Nei presidi ospedalieri con numero di posti letto limitato la realizzazione dei dipartimenti di area funzionale trova un limite nella scarsità delle discipline che qui in genere operano. In tali contesti si può immaginare una sola struttura dipartimentale (dipartimento di presidio), con aree di degenza, sempre organizzate secondo il modello dell'assistenza per intensità di cura, di tipo medico-chirurgico, ove il medico Internista continui a svolgere la funzione di principale gestore del paziente.

\section{Bibliografia}

1. Comitato Nazionale Per La Bioetica. Scopi, limiti e rischi della medicina. Roma: Presidenza Del Consiglio Dei Ministri, Dipartimento Per L'Informazione e l'Editoria; 2003.

2. De Angelis V. La logica della complessità. Introduzione alle teorie dei sistemi. Milano: Mondadori; 1996.

3. Morin E. I sette saperi necessari all'educazione del futuro. Milano: Cortina; 2001.

4. Micossi P. Il ruolo dell'ospedale del futuro. In: Falcitelli N, Trabucchi M, Vanara F, (a cura di). Rapporto Sanità 2000: documento introduttivo. Rapporto Sanità 2000. L'ospedale del futuro. Bologna: il Mulino; 2000.

5. Federspil G, Giaretta P, Moriggi S. Filosofia della medicina. Milano: Cortina; 2008. 
6. Federspil G. I fondamenti del metodo in medicina clinica e sperimentale. Padova: Piccin; 1980.

7. Scandellari C. La strategia della diagnosi. Padova: Piccin; 1981.

8. Cicchetti A. L'organizzazione dell'ospedale. Fra tradizione e strategie per il futuro. Milano: Vita e Pensiero; 2002.
9. Nardi R, Scanelli G, Borioni D, et al. The assessment of complexity in internal medicine patients. The FADOI Medicomplex Study. Eur J Intern Med 2007;18:283-7.

10. Nardi R, Arienti V, Nozzoli C, Mazzone A. Organizzazione dell'ospedale per intensità di cure: gli errori da evitare. Ital J Med 2012;6:1-13. 


\title{
I modelli organizzativi per l'assistenza territoriale primaria nel Servizio Sanitario Nazionale: evidenze ed intervento specialistico
}

\author{
Davide Croce, ${ }^{1,2}$ Antonino Mazzone ${ }^{3}$ \\ ${ }^{1}$ Centro di ricerca in economia e management in sanità e nel sociale (CREMS), Università C. Cattaneo LIUC, Castellanza \\ (VA), Italia; ${ }^{2}$ Department of Community Health, Faculty of Health Sciences, University of the Witwatersrand, \\ Johannesburg, South Africa; ${ }^{3}$ Dipartimento Area Medica, AO Legnano (MI), Italia
}

\section{RIASSUNTO}

La stagnazione del finanziamento pubblico al Sistema Sanitario Nazionale senza l'accompagnamento di una modifica dell'organizzazione dell'assistenza sanitaria (con spostamento dell'asse di cura) provocherà a breve una diminuzione del livello del servizio in quanto le richieste di risorse sono crescenti annualmente (ogni anno cresce il numero di assistititi che cronicizzano per diabete, HIV, cardiovascolare solo per tre esempi). La L. Balduzzi ha aggiunto al modello organizzativo di assistenza primaria proposto da oltre 10 anni alcune caratterizzazioni organizzative, con l'assistenza a team obbligatoria quando il $30 \%$ degli assistiti è affetto da patologie croniche [450 per medico di medicina generale (MMG)]. Tuttavia non è ancora focalizzato il rapporto tra medico specialista, MMG e assistito cronico, rapporto contrassegnato dalla continuità e dal trasferimento del focus assistenziale dalla malattia al malato. Ragionare sui meccanismi operativi del modello sanitario è necessario per salvaguardare libertà di scelta ma anche efficacia dell'intervento, in una visione olistica del tema tipica della Medicina Interna.

\section{Introduzione: i trends dei Sistemi Sanitari Nazionali}

Tutti i servizi ed i sistemi sanitari del mondo nei Paesi ad economie sviluppate sono alle prese con le stesse problematiche: cambiamento demografico, crescita delle aspettative di salute dei cittadini e delle comunità, nuove tecnologie sanitarie a disposizione. ${ }^{1}$ Alcune sono determinanti di trends (aspettative, nuove tecnologie sanitarie), altre sono conseguenze dei determinanti (aumento delle aspettative di vita che composto con atteggiamenti sociali comporta il cambiamento demografico) che hanno come risultato la crescita della spesa sanitaria totale.

Il contesto economico ed epidemiologico italiano è caratterizzato da un lato dalla stagnazione del finanziamento pubblico (crisi internazionale alla quale siamo esposti) e dall'altro dall'aumento della popo-

Corrispondente: Antonino Mazzone, Dipartimento Area Medica, AO di Legnano, via Papa Giovanni Paolo II 1, 20025 Legnano (MI), Italia.

E-mail: medicina2legnano@ao-legnano.it

Parole chiave: assistenza territoriale, cronicità, casa della salute, assistenza specialistica territoriale.

Articolo pubblicato secondo la Creative Commons Attribution NonCommercial 3.0 License (CC BY-NC 3.0).

CCopyright D. Croce e A. Mazzone, 2014

Licensee PAGEPress, Italy

QUADERNI - Italian Journal of Medicine 2014; 2:60-67 lazione esposta a patologie croniche. Quest'ultimo elemento è in larga massima dovuto al continuo sviluppo delle tecnologie sanitarie (e.g. farmaci, vaccini, presidi, programmi di assistenza, percorsi diagnostico terapeutico assistenziali, organizzazione dei servizi) che hanno modificato l'esito infausto di molte patologie (infettive come l'HIV, cardiovascolari come l'infarto, oncologiche come quello del colon-retto per citarne solo tre tra le principali). ${ }^{2} \mathrm{Il}$ nostro Servizio sanitario, come quello di molti altri Paesi economicamente sviluppati, è quindi un servizio che intrinsecamente vede un incremento della spesa ogni anno per il numero di nuovi cronici e per il conseguente incremento dell'aspettativa di vita e della popolazione anziana, e che quindi richiede un continuo sviluppo del finanziamento.,

Un altro trend cui l'Italia è esposta è la crescita delle aspettative di mantenimento della salute da parte della popolazione in generale, che si riverbera sulla continua richiesta di prestazioni sanitarie e la conseguente non accettazione dello stato di malattia e sue conseguenze. ${ }^{5}$ A questo trend si aggiungono i fenomeni sociali di mobilità (globalizzazione) e di condivisione comunicativa (internet) della popolazione, con l'allargamento dei confini di relazione e di informazione.

Infine la frammentazione e la disomogeneità dell'offerta sociale e sanitaria dei servizi sul territorio identifica la nostra assistenza. Anche per chi frequenta assiduamente il Sistema Sanitario Nazionale (SSN) non è facilmente identificabile il coordinamento complessivo dei servizi e la programmazione dei percorsi di assistenza. L'offerta assistenziale di solito non risponde in modo integrato ai bisogni dell'utenza (nei tre ambiti 
socio-assistenziale, socio-sanitario e sanitario), e ne consegue che, oltre alla inefficienza dell'assistenza, il rischio di sovrapposizioni di servizi è elevato.

La discontinuità dei servizi tra ospedale e territorio e tra i diversi operatori del territorio nasce anche da un rapporto tra professionisti non sempre ideale e collaborativo, ribadendo la prevalenza degli aspetti di comportamento rispetto a quelli organizzativi nei servizi alla persona. ${ }^{6}$

\section{La cronicità e le sue conseguenze sul Sistema Sanitario Nazionale}

Gli assistiti anziani hanno un costo di assistenza sanitaria superiore rispetto agli assistiti di tutte le altre categorie (infanti, giovani, adulti, per qualunque genere $)^{7}$ e necessitano di un servizio distribuito sul territorio ed in prossimità del domicilio (fenomeno noto come spostamento del baricentro di cura).

Nel 2012 gli assistiti definibili come cronici in Regione Lombardia in base ai dati di esenzione e di classificazione nella Banca Dati Assistito erano il 31,8\% dei 10.157 .474 assistiti totali $^{8}$ e la loro spesa complessiva era pari al 79,6\% del totale per le voci ricoveri, farmaceutica territoriale, ambulatoriale e file F. Il dato enuclea drammaticamente il ruolo ed il peso che la popolazione cronica ha ormai assunto nel nostro Paese, ed evidenzia la necessità di riprogettare i Servizi Sanitari Regionali su questo target. ${ }^{4}$

La Tabella 1 compara le caratteristiche ed i bisogni della patologia acuta e delle patologia cronica).

Al fine di garantire un appropriato livello di salute occorre assicurare all'assistito cronico un modello di presa in carico proattivo, integrato e multidimensionale. La difficoltà di assistere un paziente cronico è infatti la sua complessiva presa in carico da parte di un servizio sanitario articolato, frammentato e spesso disomogeneo sul territorio [in una dualità di governo tra medico specialista e medico di medicina generale $(\mathrm{MMG})]$. Inoltre la crescita dell'età comporta spesso la compresenza di più patologie e la crescita di una ulteriore dimensione, la fragilità.

La fragilità è un termine non univoco, nonostante ampio spazio sia stato ad esso dedicato dalla letteratura scientifica, con particolare riferimento alla sua misura oggettiva. Se riferito alla salute esso indica riduzione sia delle riserve funzionali sia della resistenza agli stressors, risultante dal declino cumulativo dei sistemi fisiologici che causano vulnerabilità e conseguenze avverse, ${ }^{9,10}$ se riferito al bio-psico-sociale viene definito come uno stato dinamico che colpisce un individuo che sperimenta perdite in uno o più domini funzionali (fisico, psichico, sociale), che aumentano il rischio di risultati avversi per la salute. ${ }^{11}$

Nei nostri assistiti cronici, contraddistinti dall'età

Tabella 1. Comparazione tra dimensioni della patologia acuta e della patologia cronica (rielaborazione degli autori su tabella di Agnello M. Presentazione della sperimentazione CreG in Regione Lombardia. Regione Lombardia; 2013).

\begin{tabular}{lcc}
\hline & Patologia acuta & Patologia cronica \\
\hline Paziente & Tutte le età & Ina patologia (alla volta) \\
\hline Esordio & Rapido & Più patologie \\
\hline Percorso di malattia & Non programmabile & Progressivo \\
\hline Durata & Breve & Prevedibile e programmabile \\
\hline Esito & Risolve in genere con la guarigione & Indeterminata \\
\hline Cura & Richiede alta specializzazione & Raramente esita in guarigione \\
\hline Atteggiamento & Ansia per la guarigione & Calo di attenzione e difficoltà di aderenza alle indicazioni sanitarie \\
\hline Compartecipazione alla spesa (ticket) & Dovuto & Esente \\
\hline Referente clinico & Uno specialista & Almeno uno specialista, caregiver e MMG \\
\hline Fragilità (clinica e sociale) & Talvolta presente & Spesso presente anche per età \\
\hline Focus clinico & Malattia & Malato \\
\hline Coinvolgimento paziente & Consenso informato & Partecipazione \\
\hline Atteggiamento clinico & Fare tutto il possibile & Ragionare sul lungo periodo \\
\hline Attività principale & Procedura & Processo \\
\hline Competenze cliniche & Iperspecializzazione & Multidisciplinarietà \\
\hline Sviluppo conoscenze & Ricerca & Ricerca organizzativa \\
\hline
\end{tabular}

MMG, medico di medicina generale. 
avanzata (Figura 1), è evidente la necessità di affiancare al modello di presa in carico sanitaria un intervento proattivo sui pazienti fragili, introducendo accanto alla dimensione clinica la dimensione sociale se necessaria. Ciò comporta una integrazione multidisciplinare dei team assistenziali e l'uso di scale di valutazione per velocizzare ed economizzare l'identificazione del bisogno.

Se la chiave di volta dell'efficientamento dell'assistenza per la patologia per acuti è stata il pagamento a prestazione (ampiamente diffusa ormai in tutto il mondo), l'approccio al paziente cronico chiede un modello di rimborso onnicomprensivo, basato sullo stato di salute. L'evidence-based case rate (ECR $)^{12}$ determina non solo le risorse complessive necessarie a gestire una determinata patologia, sia essa acuta o cronica e multidimensionale, ma anche le condizioni degli assistiti. L'ECR rappresenta, in altre parole, il costo al sistema del trattamento del paziente affetto da una determinata patologia, in base a variabili legate alla patologia stessa e al paziente. L'ECR, la cui applicazione logica in Lombardia è stata chiamata chronic related group $(\mathrm{CReG})$, scoraggia l'aumento della remunerazione basato sull'aumento dei volumi di servizio, a favore di una gestione clinica appropriata e distribuita sulle due componenti - territoriale e ospedaliera - da cui è calcolabile il costo presunto reale della prestazione.
Nei pazienti cronici proprio perché manca la presa in carico globale occorre definire, monitorare e valutare obiettivi di risultati di efficacia terapeutica e di costo. È un concetto di difficile applicazione pratica che andrà affrontato con approccio pragmatico e le necessarie semplificazioni (come in tutte le modalità di rimborso sanitario, si pensi al diagnosis-related group).

\section{Il nuovo ruolo della medicina di base}

Nell'assistenza primaria il ruolo centrale nel SSN italiano è sempre stato assegnato al Medico di Medicina Generale, agente garante dello stato di salute dei suoi assistiti (massimo 1500) e per un lungo periodo gatekeeper dell'accesso al servizio. Il modello ha funzionato egregiamente per anni e pur in una solitudine che evidenziava grandi differenze di comportamento e di performance tra i MMG di una stessa azienda anche in territori omogenei (con percezione del ruolo diversa), i risultati in una popolazione giovane sono stati ottimi. ${ }^{13}$

La continua crescita dei pazienti cronici, oltre il $30 \%$ ormai del totale degli assistiti (dati Lombardia), mette tuttavia in discussione non il ruolo del MMG ma il modello organizzativo: un professionista singolo, con limitati mezzi organizzativi, che deve fronteggiare oggi 450 pazienti cronici è probabilmente carente. È impos-

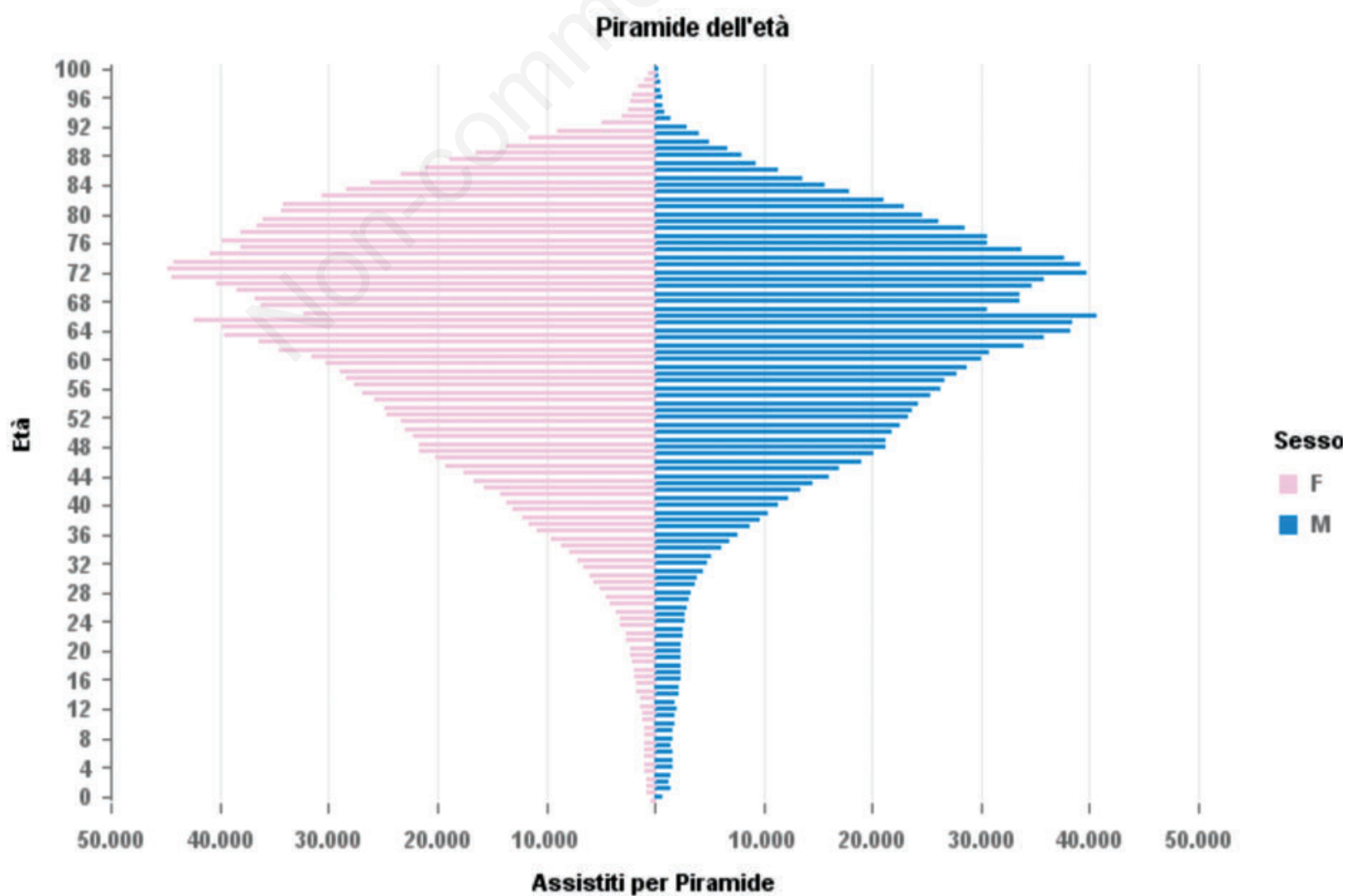

Figura 1. Età della popolazione cronica in Lombardia anno 2012. Fonte: Direzione Generale Sanità. 
sibile affrontare attraverso una presa in carico globale ed effettiva dell'assistito il carico di lavoro dato anche dai soli pazienti cronici (tralasciando per il momento gli altri 1050 assistiti, che comunque hanno esigenze temporanee di assistenza) e dalle relazioni che questo comporta (ogni paziente ha un differente medico specialista di riferimento ed il MMG avrebbe la necessità di relazionarsi con tutti loro).

Nella Tabella 2 vengono riportati gli anni di anzianità dalla laurea dei MMG attivi in Italia.

Nei prossimi anni la gran parte dei medici di medicina generale oggi in attività terminerà la propria attività lavorativa con il SSN per quiescenza, ed il servizio perderà un know-how professionale inserendo giovani medici con approcci lavorativi (ed atteggiamenti) differenti.

Se il singolo non può più essere il solo responsabile della medicina primaria, il MMG potrebbe essere il solo regista (od uno) delle attività di presa in carico, attivando al bisogno le figure professionali e gli strumenti di prevenzione, diagnosi e cura necessari, in un nuovo ruolo di coordinamento e di governo clinico, superando la presa in carico individuale dell'assistito attraverso un'assistenza a team multidimensionale. Ad esempio l'infermiere di comunità, inserito nel team come care manager, può identificare le esigenze assistenziali (assistenza domiciliare, controllo dei parametri, educazione sanitaria, ...) ma anche le necessità degli assistiti in termine di prevenzione, visitando ad intervalli regolari gli assistiti più anziani ed intervenendo sugli assistiti con cognizione di risk adjusted. Anche il fisioterapista potrebbe avere compiti nelle attività di riabilitazione (oggi 1/5 delle giornate di degenza sanitaria acuta in Lombardia sono per riabilitazione).

I medici in generale dovrebbero anche affrontare la crescente criticità che assumono le rappresentazioni delle attività sanitarie (sia economiche che di gestione della patologia) attraverso l'enorme mole di dati raccolti in sanità. La verifica di appropriatezza è auspicabile sia ex ante [e.g. attraverso le linee guida (LG) ed $\mathrm{i}$ percorsi diagnostici terapeutici assistenziali (PDTA)], sia ex post (e.g. leggendo i percorsi e le aderenze agli stessi che il paziente ha effettivamente realizzato nel periodo). Il sistema informativo, anche con l'uso ad esempio del fascicolo elettronico del paziente, diventa praticamente indispensabile nelle aree remote,

Tabella 2. Anzianità di laurea dei medici di medicina generale, per Regione - Anno 2011. Fonte: Ministero della Salute.

\begin{tabular}{|c|c|c|c|c|c|c|}
\hline Regione & Da 0 a 6 anni & Da 6 a 13 anni & Da 13 a 20 anni & Da 20 a 27 anni & Oltre 27 anni & Totale \\
\hline Piemonte & 18 & 78 & 569 & 1005 & 1651 & 3321 \\
\hline Valle d'Aosta & 0 & 1 & 15 & 20 & 54 & 90 \\
\hline Lombardia & 0 & 95 & 742 & 1980 & 3852 & 6669 \\
\hline Prov. Auton. Bolzano & 2 & 18 & 55 & 66 & 135 & 276 \\
\hline Prov. Auton. Trento & 0 & 5 & 63 & 110 & 206 & 384 \\
\hline Veneto & 1 & 69 & 493 & 995 & 1884 & 3442 \\
\hline Friuli Venezia Giulia & 1 & 18 & 109 & 309 & 522 & 959 \\
\hline Liguria & 0 & 12 & 150 & 350 & 772 & 1284 \\
\hline Emilia Romagna & 2 & 55 & 273 & 763 & 2090 & 3183 \\
\hline Toscana & 5 & 69 & 199 & 707 & 1951 & 2931 \\
\hline Umbria & 2 & 10 & 66 & 195 & 487 & 760 \\
\hline Marche & 3 & 41 & 130 & 304 & 740 & 1218 \\
\hline Lazio & 0 & 102 & 636 & 1533 & 2591 & 4862 \\
\hline Abruzzo & 0 & 6 & 80 & 234 & 799 & 1119 \\
\hline Molise & 0 & 0 & 8 & 50 & 207 & 265 \\
\hline Campania & 32 & 52 & 412 & 1293 & 2559 & 4348 \\
\hline Puglia & 2 & 16 & 363 & 1072 & 1853 & 3306 \\
\hline Basilicata & 0 & 1 & 39 & 135 & 332 & 507 \\
\hline Calabria & 18 & 16 & 74 & 396 & 1064 & 1568 \\
\hline Sicilia & 12 & 31 & 357 & 1269 & 2554 & 4223 \\
\hline Sardegna & 0 & 8 & 202 & 474 & 662 & 1346 \\
\hline Italia & 98 & 703 & 5035 & 13.260 & 26.965 & 46.061 \\
\hline
\end{tabular}


dove le distanze non favoriscono quel lavoro in team, indispensabile al paziente cronico e che può essere surrogato dalle autostrade informative elettroniche.

Uno dei segnali di una non ottimale presa in carico dei servizi territoriali è per altro rappresentata dalla tipologia delle prestazioni dei codici bianchi e verdi che accedono al pronto soccorso in Regione Lombardia (si riporta a titolo di esempio la Tabella 3).

\section{I centri territoriali o case della salute: elemento organizzativo utile?}

Il ruolo che il MMG dovrebbe assumere necessita di un luogo fisico per offrire assistenza continua ed organizzata in prossimità del domicilio degli utenti. La L. Balduzzi ${ }^{14}$ ha introdotto le Unità complesse di cure primarie (UCCP) come Aggregazioni funzionali territoriali ed ha regolato alcune delle caratteristiche delle stesse.

Le UCCP (o Centri Territoriali o Case della Salute) attraverso l'aggregazione di MMG (circa 10) ed altri professionisti e specialisti, realizzerebbero il punto di accesso riconoscibile al sistema della salute. Essi non solo rappresentano il luogo di incontro tra le diverse figure professionali e un elemento di integrazione in una visione sistemica di un continuum tra l'ospedale e il territorio, ma realizzano quella presa in carico proattiva e globale che oggi manca al SSN, garantendo l'uso di buone pratiche in modo da facilitare e ridurre gli accessi, snellire le procedure, integrare e mettere in comunicazione le varie funzioni specialistiche con riduzione di tempo delle prestazioni erogate, migliorando l'informazione e l'educazione al paziente e al familiare. Per questo devono avere tempi di apertura compatibili con i ritmi della vita lavorativa e aperti alle esigenze di una società multiculturale, per arrivare ad essere identificati come luoghi di salute e non come uffici amministrativi.

La Tabella 2 riporta il totale dei MMG in servizio nel 2011 in Italia: circa 46.000 e quindi le aggregazioni dovrebbero essere poco meno di 5000. Uno sforzo grande ma non impossibile se congiunto con gli Enti Locali e le cooperative di MMG. Con un nodo critico: chi gestisce le Case della Salute?

\footnotetext{
a La Casa della salute è da intendersi come la sede pubblica dove trovano allocazione, in uno stesso spazio fisico, i servizi territoriali che erogano prestazioni sanitarie, ivi compresi gli ambulatori di Medicina Generale e Specialistica ambulatoriale, e sociali per una determinata e programmata porzione di popolazione. In essa si realizza la prevenzione per tutto l'arco della vita e la comunità locale si organizza per la promozione della salute e del benessere sociale (Ministero della Salute, http:/www.salute.gov.it/portale/ temi/p2_6.jsp?lingua $=$ italiano\&id $=822 \&$ area $=$ Cure $\% 20$ primarie \&menu=casa)
}

Le UCCP hanno avuto uno sviluppo storico senza soluzione di continuità durato oltre 10 anni (da Gruppi di cure primarie a Unità territoriali di assistenza primaria, da Casa della Salute a Centri sanitari territoriali) che ha permesso al progetto di esprimere compiutamente gli obiettivi abbinati agli elementi costitutivi (e.g. da sistemi di valutazione multidimensionale del bisogno standardizzati a indirizzo verso $i$ diversi nodi della rete a seconda delle esigenze rilevate, da erogazione di prestazioni sanitarie a quelle socio-sanitarie come l'assistenza domiciliare integrata $(\mathrm{ADI})$, da sanità di iniziativa ad attività fisica adattata, da invio alla riabilitazione ambulatoriale alla presa in carico riabilitativa nel centro).

Interessa qui porre in evidenza i bisogni ed i meccanismi operativi intesi come relazioni anche per minimizzare gli elementi deboli del modello: i) impossibilità di creare le UCCP nelle aree spopolate del paese (e.g. montagna e campagna); ii) resistenza al cambiamento dei professionisti; iii) strumenti di valutazione di outcomes informatizzati; iv) pagamento a prestazione o per assistito, (moltiplicazione delle attività) da trasformare in pagamento per stato di salute; v) preparazione professionale ad un modello organizzativo differente; vi) presa in carico reale; dato che gli elementi di forza (e.g. punto unico di accesso al servizio sanitario e socio-sanitario, identificabilità, pluralità di prestazioni disponibili in un unico luogo, multiprofessionalità $\mathrm{e}$ conseguenti ricadute positive sulle prestazioni, organizzazione per percorsi diagnostici terapeutici basati sulle evidenze, continuità assistenziale potenziale) sono state ad oggi ampiamente discusse.

Le aree impervie del territorio non possono accogliere il modello di aggregazione arricchita per prestazioni della medicina di base, pena l'imposizione agli assistiti dell'utilizzo estensivo dei mezzi di trasporto e quindi un peggioramento dell'assistenza offerta. I modelli di aggregazione virtuale attraverso reti informatiche possono essere comunque un surrogato al tema. Gli altri temi trasferenti negatività al modello hanno soluzioni semplici.

Un modello assistenziale organizzato sul territorio assicurerebbe inoltre una dimissione protetta dei pazienti in regime acuto che è uno dei problemi più sentiti dalle UUOO di medicina interna, anche in funzione dei ricoveri ripetuti che a volte avvengono a seguito di dimissione precoci dalle iperspecialità.

Il meccanismo operativo in grado di modificare la performance del SSN è tuttavia il legame tra il paziente, il MMG con i supporti del Centro territoriale ed il medico specialista di riferimento per la patologia cronica del paziente. Quest'ultimo entra quasi sempre come riferimento principale del paziente rispetto ai temi di salute e quindi occorre sistematizzare l'organizzazione per renderla efficace. Un MMG che si deve relazionare con gli specialisti dei suoi 450 assistiti cro- 
Tabella 3. Diagnosi dei Pronto Soccorso della Lombardia per principali accessi (primi 11 mesi del 2013).

\begin{tabular}{lcc}
\hline Diagnosi & Bianchi (\%) & Verdi (\%) \\
\hline 7242 - Lombalgia & 12,89 & 6,55 \\
\hline 9999 - Altre e non specificate complicazioni di cure mediche, non classificate altrove & 10,75 & 9,54 \\
\hline 462 - Faringite acuta & 10,51 & 4,87 \\
\hline 4659 - Infezioni acute delle vie respiratorie superiori, sede non specificata & 7,65 & 3,19 \\
\hline 7806 - Febbre & 6,11 & 5,33 \\
\hline V221 - Controllo di altra gravidanza normale & 5,50 & 5,41 \\
\hline 78096 - Dolenzia generalizzata & 5,00 & 4,60 \\
\hline 78900 - Dolore addominale sede non specificata & 4,95 & 6,69
\end{tabular}

nici se non ha una standardizzazione delle relazioni formali anche attraverso un supporto di tipo informatico per la gestione dell'assistito non sarà mai efficace (e nemmeno efficiente). Sembra quindi logico collegare rigidamente i Centri Territoriali con i presidi ospedalieri della zona in modo che siano rese semplici e formalizzate le relazioni tra chi ha la responsabilità della presa in carico (e che può condividerla), la responsabilità del supporto specialistico e chi ha la responsabilità della cura.

\section{L'organizzazione specialistica}

L'elemento più significativo per garantire l'appropriatezza del percorso di cura è il rapporto con i medici specialisti riferimento per la patologia, elemento di collegamento essenziale nella presa in carico ma poco dibattuto nella letteratura a riferimento. Spesso (o quasi sempre) nelle patologie croniche si instaura un rapporto fiduciario e continuo tra paziente e medico specialista, rapporto alimentato nel tempo dalle frequentazioni che allarga a tutto il pianeta salute questa interazione. La doppia relazione di agenzia (MMG e specialista) non costituisce di norma un problema di rapporti tra professionisti, ma al MMG viene richiesto di mantenere una relazione con decine di specialisti di diverse discipline. La complicazione nel numero dei rapporti diviene importante quando l'offerta è vasta, come nelle aree metropolitane. Una doppia presa in carico interdisciplinare può funzionare in altri contesti assistenziali (ospedalieri) come il comanagement, in ambienti culturali differenti (USA) ${ }^{\mathrm{b}}{ }^{15}$ riproducibili in Italia con grandi volontà.

b L'hospital medicine - modello organizzativo secondo cui un medico unico prende in carico il paziente all'ingresso in ospedale e lo restituisce al MMG alla dimissione - rappresenta la specialità a più rapida crescita negli USA.
La libera scelta del cittadino è uno dei valori fondanti del SSN ma occorre rispettare anche l'efficienza dell'organizzazione, con particolare riferimento all'attuale momento di difficoltà economica. La massima efficienza teorica viene raggiunta all'instaurarsi del coordinamento delle attività con meccanismi di standardizzazione che diminuiscono l'ampiezza dei rapporti.

Come nella scelta del MMG vi è una libertà vincolata al territorio di residenza ed alla disponibilità determinata sul numero di assistiti, così nel rapporto tra paziente cronico e medico di specialità andrebbe posto un vincolo territoriale (con alcune flessibilità) che consenta all'organizzazione dei servizi sanitari un livello di efficienza ed efficacia che si riverbererebbe positivamente sugli outcomes dei pazienti cronici (Figura 2).

Nel modello organizzativo lombardo della sperimentazione $\mathrm{CReG}$ il rapporto tra assistenza primaria ed assistenza specialistica è affidato in toto al gestore che acquista pacchetti di prestazioni specialistiche dai providers (e che quindi stabilizza i rapporti incentrando su di se, responsabile della presa in carico, la relazione principale medico-paziente).

Gestire pazienti cronici (definiti altrimenti come complessi) richiede buone capacità relazionali (il focus si sposta dalla malattia alla persona), strumenti (e.g. sistema informativo sanitario, fascicolo elettronico, PDTA e LG, modalità di contatto rapido) e organizzazione (inserimento in un percorso chiaro a colui che lo vive).

In un recente documento del Ministero della Salute ${ }^{16}$ si afferma che sta divenendo meno importante l'agire medico incentrato su un approccio d'organo (da malattia a terapia a guarigione) soverchiato dalla necessità di un approccio di sistema (da persona a definizione dei problemi a qualità della vita).

Da questo punto di vista l'internista è uno dei pochi specialisti che si interfaccia sui molti aspetti della cura, e quindi è capace di una visione olistica dello stato di salute ${ }^{17}$ differente dalle specialità d'organo, costruite sulla iperspecializzazione che tanto ha 


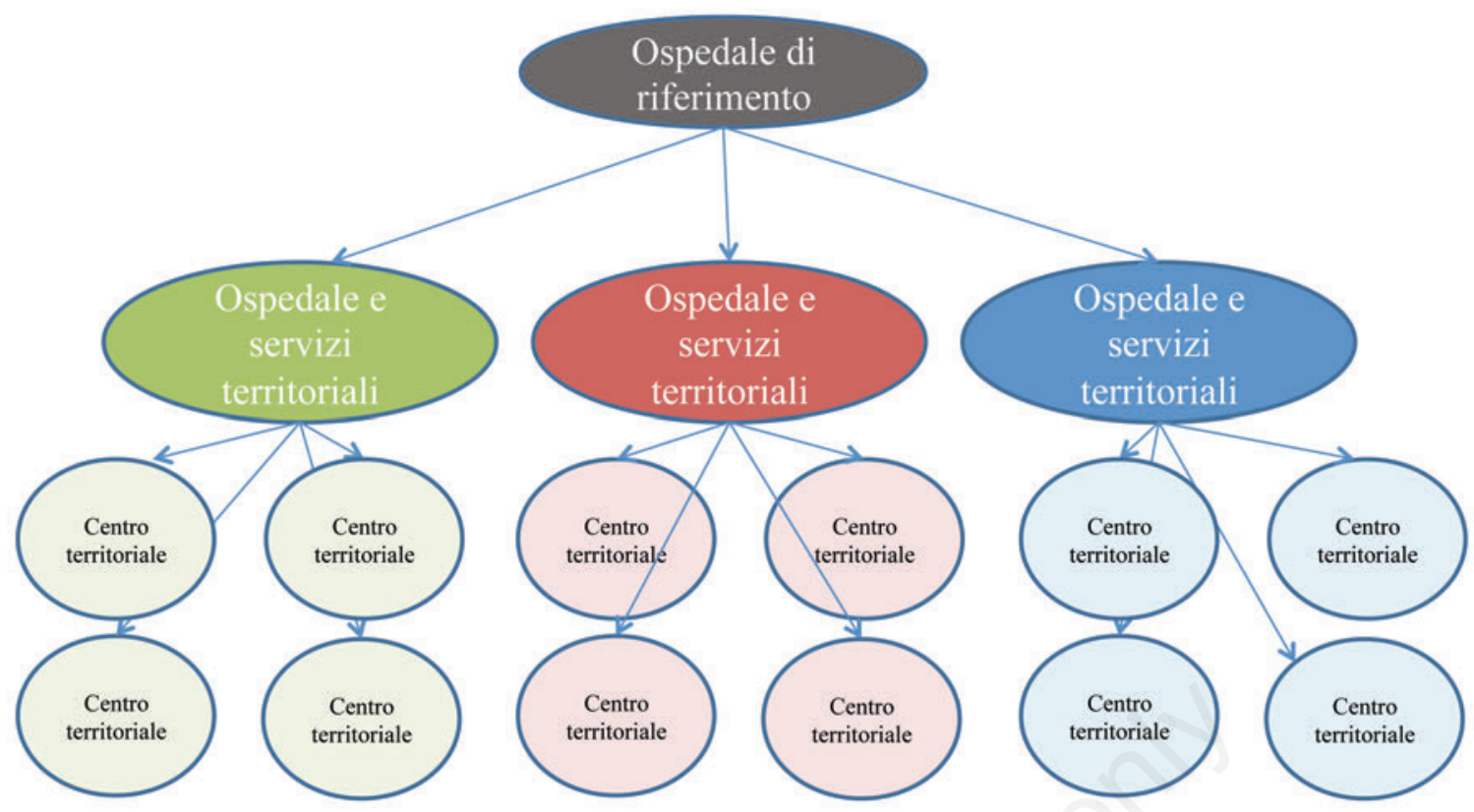

Figura 2. Modello organizzativo con riferimenti dell'assistenza specialistica ospedaliera per il paziente cronico.

portato allo sviluppo della medicina ma che si confronta oggi con le necessità del paziente anziano e complesso.

\section{Conclusioni}

La mancata crescita del finanziamento del servizio sanitario italiano ha posto in evidenza la necessità di modifica del modello di assistenza sanitaria, con particolare riferimento alla presa in carico del paziente cronico. Senza un cambio di modello organizzativo nel servizio sanitario nazionale siamo infatti destinati ad una diminuzione dei livelli dell'assistenza offerta a causa della crescita delle richieste assistenziali (ogni anno crescono in numero i pazienti cronici con tassi annuali elevati nelle patologie metaboliche e nelle cardiovascolari solo per citare due esempi) che non trovano riscontro nelle risorse finanziarie messe a disposizione.

Se la medicina primaria ha ormai sviluppato un modello di presa in carico efficace (a team), supportato dalla regolazione della L. Balduzzi e dalle sperimentazioni in varie Regioni (Toscana, Emilia Romagna, Lombardia, Veneto, Puglia tra le altre), non così si può dire del livello di rapporti immediatamente superiore, che è altrettanto importante nell'efficacia del trattamento dei pazienti cronici. Riflettere su questi modelli è indispensabile per la costruzione dei percorsi di cura e di assistenza.

Un ruolo importante nel modello sarà quello della medicina interna, che ha nell'approccio al paziente complesso un suo standard consolidato, assente in molte altre specialità cliniche e che nel prossimo futuro continuerà a crescere di importanza in una società che invecchia come quella italiana.

Lavorare in team è ormai indispensabile anche per chi ha sempre agito attraverso l'individualità delle azioni: assistere 450 pazienti cronici è estremamente complicato e probabilmente non efficiente con riferimento all'intero ciclo dell'assistenza sanitaria.

Tutti i temi che si affrontano nel trasferimento dell'asse di cura portano intrinsecamente richieste di investimento (i luoghi fisici) di formazione (capacità di estrazione e di lettura degli output riferiti al singolo assistito, capacità di lavorare in gruppo, capacità di lavorare con strumenti a distanza tra gli altri che si potrebbero elencare) e di flessibilità: i modelli proposti dovranno essere adattati alle condizioni locali (di risorse e di abitudini).

Quest'ultimo punto (investimenti in risorse, formazione, persone, anche se a livelli limitati contando sul ricambio del personale) assieme alla volontà politica di modificare, migliorandolo, il servizio è il vero punto debole di quanto dibattuto nello scritto.

\section{Bibliografia}

1. Joppi R, Pase D, Poggiani C. Italian horizon scanning project. G Ital Health Technol Assess 2013:6;11-21.

2. Rosen AB, Cutler DM, Norton DM, et al. The value of coronary heart disease care for the elderly: 1987-2002. Health Aff 2007;26:111-23.

3. Rechel B, Doyle Y, Grundy E, McKee M. How can 
health systems respond to population ageing? Geneva: World Health Organization; 2009.

4. Senin U, Cherubini A, Mecocci P. Impatto dell'invecchiamento della popolazione sull'organizzazione sociosanitaria: necessita di un nuovo modello di assistenza continuativa. Ann Ital Med Intern 2003;18:6-15.

5. Kickbush I, Maag D. Lo sviluppo della Health literacy nelle moderne società della salute Salute e Società 2007;16:185-200.

6. Anderson RA, McDaniel RR. Managing health care organizations: where professionalism meets complexity science. Health Care Manage Rev 2000;25:83-92.

7. WHO. World Health Statistic 2008. Geneva: WHO library; 2008.

8. Regione Lombardia. Deliberazione Giunta regionale d.g.r. D.g.r. 26 ottobre 2012 - n. IX/4334 Determinazioni in ordine alla gestione del servizio socio sanitario regionale per l'esercizio 2013. B.U. Lombardia n. 2, 09/01/2013. Disponibile su: http://www.consultazioniburl.servizirl.it/ConsultazioneBurl/temp/140371267019 08347276590954978618.pdf\#page=21

9. Fried LP, Walston J. Frailty and failure to thrive. In: Hazzard WR, Blass JP, Ettinger WH Jr, et al., eds. Principles of geriatric medicine and gerontology. 4th ed. New York: McGraw-Hill; 1999. pp 1387-1402.

10. Fried LP, Tangen CM, Walston J, et al. Frailty in older adults: Evidence for a phenotype. J Gerontol A Biol Med Sci 2001;56:M146-57.

11. Gobbens RJ, Luijkx KG, Wijnen-Sponselee MT, Schols JM. In search of an integral conceptual definition of frailty: opinions of experts. J Am Med Dir Assoc 2010; 11:338-43.

12. De Brantes F, Camillus JA. Evidence-informed case rates: a new health care payment model. The Commonwealth Fund; April 2007. Available from: http://www. commonwealthfund.org/Publications/FundReports/ 2007/Apr/Evidence-Informed-Case-Rates-ANew-Health-Care-Payment-Model.aspx

13. Longo F, Vendramini E. Il budget e la medicina generale. New York: McGraw-Hill; 2004.

14. Ministero della Giustizia. Testo del decreto-legge 13 settembre 2012, n. 158, (pubblicato nella Gazzetta Ufficiale 13 settembre 2012, n. 214), coordinato con la legge di conversione 8 novembre 2012, n. 189 (in questo stesso supplemento ordinario alla pag. 1), recante: «Disposizioni urgenti per promuovere lo sviluppo del Paese mediante un piu' alto livello di tutela della salute.». G.U. n. 263, 10/11/2012 - Suppl. Ordinario n. 201. Available from: http://www.altalex.com/index.php?idnot=58574

15. Wachter RM, Goldman L. The hospitalist movement 5 years later. JAMA 2002;287:487-94.

16. Ministero della Salute. Criteri di appropriatezza clinica, tecnologica e strutturale nell'assistenza del paziente complesso. Quaderni del Ministero della Salute N. 23/2013. Available from: http://www.quadernidellasalute.it/download/download/23-settembre-ottobre-2013quaderno.pdf

17. Nardi R, Scanelli G, Borioni D, et al. The assessment of complexity in internal medicine patients. The FADOI Medicomplex Study. Eur J Intern Med 2007;18:283-7. 


\section{La Medicina Interna ospedaliera: tra complessità ed intensità assistenziale verso una medicina sostenibile}

Andrea Fontanella

Dipartimento di Medicina, Ospedale Madonna Del Buon Consiglio Fatebenefratelli, Napoli, Italia

I tradizionali modelli medici, così come sono insegnati nelle nostre università, sono fatti per essere lineari, restrittivi e super semplificati, in assoluto contrasto con la realtà dei pazienti, prevalentemente anziani, degenti nei nostri reparti di Medicina Interna. Tutto si basa sul tradizionale, storico approccio scientifico del riduzionismo: l'anatomia si occupa del corpo, la fisiologia degli organi, la biologia delle cellule, la biologia molecolare delle proteine e dei geni. Abbiamo scomposto l'Universo e ora non sappiamo più come rimontarlo. Per dirla con le parole di Barabási, scienziato Ungherese, che introdusse il concetto della Network Medicine, ci stiamo comportando come il bambino che, dopo aver smontato il suo giocattolo preferito, per vedere come funziona, piange disperatamente perché non sa più come rimetterlo a posto per farlo funzionare di nuovo. Nel concetto del Network-based approach to human disease, le malattie sorgono come conseguenza del fatto che una o più reti biologiche nell'organo interessato divengono patologicamente alterate, attraverso cambiamenti patologici genetici e/o ambientali.

La complessità dei nostri pazienti, d'altronde, non può essere ridotta a un insieme di patologie e di terapie, ma deve intendersi come un intreccio e sovrapposizione d'influenze reciproche, in uno stesso individuo, di due o più sistemi: patologici, terapeutici, psicologici, socio-economici, familiari, culturali, assistenziali, ecc. Tutto ciò richiede una valutazione multidimensionale dei problemi in causa e, a tutt'oggi, non vi è ancora un sistema univoco per definire e $p e-$ sare il paziente complesso. Questo sarà lo sforzo e

Corrispondente: Andrea Fontanella, Dipartimento di Medicina, Ospedale Madonna Del Buon Consiglio Fatebenefratelli, Napoli, Italia.

E-mail: andreafontanella52@gmail.com

Articolo pubblicato secondo la Creative Commons Attribution NonCommercial 3.0 License (CC BY-NC 3.0).

CC Copyright A. Fontanella, 2014

Licensee PAGEPress, Italy

QUADERNI - Italian Journal of Medicine 2014; 2:68-69 l'impegno della FADOI per i prossimi anni, al fine di effettuare scelte appropriate per il trattamento del paziente e non della patologia, anche allo scopo di ottimizzare le risorse.

Gli strumenti di tali valutazioni dovranno tenere in conto una serie di domini quali: comorbilità, stabilità clinica, fragilità sociale, disfunzione cognitiva, depressione, dipendenza funzionale, nutrizione, ulcere da pressione, dolore, aderenza alla terapia, politerapie, ecc. che permettano una più appropriata definizione del profilo di complessità del paziente ricoverato, contribuendo ad un miglioramento della qualità dell'assistenza intra-ospedaliera, indirizzandola verso livelli di intensità individualizzati.

Contemporaneamente, esso potrà generare informazioni utili a gestire la continuità assistenziale nella delicata transizione ospedale-territorio, ottimizzando le potenzialità dei sistemi sanitari dedicati alla postacuzie e alla cronicità.

Naturalmente un primo passo nella definizione della complessità del paziente medico è quello di stratificarlo per severità di patologia, anche al fine di una precisa collocazione, in base alla diversificazione dei livelli di cura adeguati e necessari al grado di malattia. A tale scopo si è cercato di validare score di facile esecuzione anche da parte di personale non medico, al fine di identificare le criticità e favorire un'eventuale allocazione dei pazienti nei setting più idonei al grado d'instabilità. Il più noto score proposto e variamente utilizzato a questi scopi è stato il modified early warning score (MEWS). Il vero limite del MEWS è che esso esplora l'instabilità clinica dei pazienti ma è assolutamente inadeguato a valutarne la complessità secondo le diverse dimensioni e, inoltre, considera solamente parametri di base e nessun elemento clinico. È ben noto come qualunque paziente possa presentare condizioni (vedi ipertermia) o patologie, in grado di fare improvvisamente precipitare la situazione clinica, anche se all'ingresso il paziente aveva un punteggio MEWS di tutta tranquillità. Pertanto il più efficace strumento di valutazione dell'instabilizzazione dei pazienti è quello dell'osservazione clinica, da ripetersi più volte nell'arco della giornata.

Nel contesto dell'attuale riorganizzazione ospedaliera, secondo il modello di aggregazione dipartimen- 
tale, è necessaria la ricerca di nuove forme organizzative, quali l'ospedale basato sull'intensità di cure, UO di tipo dipartimentale, gestione integrata ospedale-territorio per pazienti affetti da patologie croniche. In tale ambito la Medicina Interna può esercitare un ruolo determinante facendosi carico del coordinamento delle cure multi professionali.

In quest'area vasta, la complessità reca il suo contributo metodologico alla sanità in generale e alla gestione dell'assistenza, così come al contesto clinico e alla stessa formazione medica. La complessa natura della sanità odierna deve adeguarsi alla crescente articolazione della domanda, così come alla differenziazione dei quadri nosografici sempre più inaspettati, $\mathrm{e}$ su queste basi realizzare risposte flessibili ai modelli e alle opportunità emergenti.

La complessità nella gestione dell'assistenza può quindi essere sostenuta dal Clinico Medico, dal Medico Internista, capace, all'interno di un sistema adattativo complesso, di svolgere un'azione di coordinamento, d'integrazione, di cooperazione fra le diverse parti che agiscono per ambiti specifici di specializzazione settoriale.

Anche dal punto di vista retributivo, per quanto concerne la riorganizzazione dei diagnosis-related groups (DRGs), il sistema ha la necessità di cambiare. Oggi i DRGs hanno la pretesa di voler quantificare economicamente l'assorbimento di risorse e quindi di remunerare ciascun episodio di ricovero. È sempre più necessario introdurre il concetto che il malato cronico debba essere pagato per stato di salute e non per prestazione, pena la moltiplicazione delle stesse in una spirale di tipo statunitense.

In quest'ambito diventa veramente difficile essere saggi. L'attuale condizione di crisi economica, nazionale e mondiale, impone la necessità di fare delle scelte, anche allo scopo di poter continuare a mantenere il wel- fare. Non sempre fare di più significa fare meglio, anzi, spesso, fare di più vuol dire: spreco di risorse, occorrenza d'incidentalomi, indagini inutili, effetti iatrogenici e quant'altro. È, pertanto, indispensabile ponderare le scelte, considerare le necessità del paziente, cercare modelli che rispondano ai reali bisogni di chi assistiamo. Per questo il tema centrale di questo progetto è legato a un movimento culturale che sta prendendo vita in Italia e che vuole far riflettere tutti i professionisti della salute sui propri orientamenti, indirizzandoli verso una medicina saggia e sostenibile.

Già nel lontano 1956 il grande filosofo Bertrand Russel (1872-1970) nel suo scritto Conoscenza e saggezza (Knowledge and Wisdom), espone idee, tuttora condivisibili, riguardo alla necessità che la saggezza, difficile equilibrio di responsabilità, altruismo, rigore, senso del limite, imparzialità, si debba accompagnare al progresso della conoscenza. La frase recita: $A$ ogni accrescimento della conoscenza e della tecnica, la saggezza diviene più necessaria, poiché ognuno di questi accrescimenti aumenta la nostra capacità di attuare i nostri scopi, e perciò aumenta la nostra capacità di far del male, se i nostri scopi non sono saggi.

Ovvero leggendo la frase al contrario: Il problema dei tempi attuali è che la velocità di progressione della tecnica, che si autoalimenta, è molto superiore a quella del pensiero. Il risultato è che le conoscenze aumentano e la saggezza diminuisce!

La parola d'ordine, quindi, della Medicina del nuovo millennio è appropriatezza, diagnostica e terapeutica. Sarà proprio l'appropriatezza che, evitando gli errori in difetto e in eccesso, permetterà di migliorare l'efficacia e l'efficienza dell'intervento medico e, quindi, di arginare l'inutile levitare della spesa sanitaria e di permettere, cosi, di ridurre i temuti e dannosi tagli indiscriminati, cui le Regioni sono costrette dai piani di rientro. 


\section{LINEE GUIDA PER GLI AUTORI}

I Quaderni dell'Italian Journal of Medicine (Quaderni ITJM), costituiscono una collana supplementare solo online annessa alla rivista Italian Journal of Medicine contenente lavori solo in lingua italiana.

I Quaderni ITJM pubblicano:

- Monografie ad hoc su temi di particolare rilevanza scientifica a cura della Commissione FADOI Giovani, coordinata dalla Dr.ssa Paola Gnerre.

- Traduzioni in italiano di alcuni lavori pubblicati sui numeri standard dell'Italian Journal of Medicine, di particolare interesse per la comunità scientifica. La selezione è a cura dell'Editor-inChief, Prof. Roberto Nardi.

\section{STESURA DEI LAVORI}

I lavori dovranno essere redatti in modo conforme alle linee guida sotto riportate:

- I manoscritti devono essere scritti interamente in lingua italiana, su documento di Word, con carattere Times New Roman/Arial, dimensione 12, formato A4, interlinea doppia e margini $2,54 \mathrm{~cm}$. Parole totali: $\max 4000 ;$ Sommario/Abstract: $\max 250$ parole; Bibliografia: $\min 40$ voci; Tabelle e Figure: $3 / 5$ totali (le tabelle non devono superare n. 1 pagina del documento in Word).

- La strutturazione del contenuto deve attenersi agli standard internazionali per la Rassegna (Review): i) Abstract riassuntivo dell' 'intero lavoro; ii) Introduzione al tema trattato; iii) Criteri e strumenti di ricerca (criteri di inclusione/esclusione, banche dati consultate, ...); iv) i successivi paragrafi devono illustrare le più recenti scoperte scientifiche nel settore; v) Conclusioni; vi) Bibliografia.

- La prima pagina deve riportare: i) titolo (in stampatello minuscolo), senza acronimi; ii) nome e cognome per esteso di ciascun autore; iii) affiliazione(i) di ciascun autore, numerate con numeri arabi; iv) eventuali ringraziamenti; v) nome e indirizzo postale completi dell'autore corrispondente, corredati da telefono, fax, e-mail; vi) da 3 a 5 parole chiave, separate da virgola. La seconda pagina può riportare: i) contributi degli autori, e.g. informazioni relative a contributi sostanziali delle persone coinvolte nello studio (http://www.icmje.org/\#author); ii) dichiarazione relativa a potenziali conflitti d'interesse; iii) ulteriori informazioni (e.g. fondi, esposizioni durante conferenze...).

- In caso di utilizzo di tabelle, queste devono essere tutte numerate con numeri arabi e citate nel testo in ordine consecutivo (e.g. NON nominare le tabelle come Tabella $1 \mathrm{~A}, 1 \mathrm{~B}, \ldots$ o $1.0,1.1, \ldots$.). Le tabelle devono essere presentate in formato editabile. Ciascuna tabella deve essere corredata da una breve didascalia; in caso di abbreviazioni, riportare una nota a piè di CIASCUNA tabella che spieghi TUTTE le abbreviazioni presenti in ognuna.

- In caso di utilizzo di figure, queste devono essere inviate in formato tiff o .jpg, allegate al manoscritto in singoli files, secondo le seguenti specifiche:

i) a colori (salvate in modalità CMYK): minimo 300 dpi di risoluzione;

ii) in bianco e nero: minimo 600 dpi di risoluzione;

iii) minimo $17,5 \mathrm{~cm}$ di larghezza.

Ciascuna figura deve essere corredata da una breve didascalia.

NB: In caso di Tabelle/Figure riprese e/o modificate da altri lavori già pubblicati, sarà cura degli autori accertarsi se tali materiali siano o meno coperti da copyright e procurarsi i permessi necessari per la riproduzione. Tali permessi dovranno essere allegati alla versione definitiva del lavoro. L'ufficio editoriale si riserva la facoltà di rimuovere Tabelle/Figure coperte da copyright, se sprovviste dei necessari permessi

- In caso di utilizzo di abbreviazioni, la prima volta che esse sono citate è necessario scrivere per esteso la definizione+abbreviazione tra parentesi tonde [e.g. risonanza magnetica $(\mathrm{RMN})]$, a seguire si dovrà riportare solo l'abbreviazione (unica eccezione: nei titoli e nelle didascalie di tabelle e figure NON si utilizzano abbreviazioni).

\section{BIBLIOGRAFIA}

Le voci bibliografiche devono essere formattate secondo lo stile Vancouver.

Nella sezione Bibliografia, le voci bibliografiche devono essere numerate consecutivamente nell'ordine in cui appaiono per la prima volta nel testo (NON in ordine alfabetico) e, nel testo, devono essere indicate con numeri arabi in apice. Voci bibliografiche riferite a comunicazioni personali o dati non pubblicati devono essere incorporate nel testo e NON inserite tra le voci numerate [e.g. (Wright 2011, dati non pubblicati) o (Wright 2011, comunicazione personale)].

Le voci bibliografiche nella sezione Bibliografia devono tassativamente essere preparate come segue:

i) più di 3 autori, citare 3 autori, et al. Se il lavoro contiene solo 4 autori, citarli tutti e 4 ;

ii) titolo del lavoro in stampatello minuscolo;

iii) nome della rivista, senza punti, abbreviato secondo gli standard internazionali; in caso di dubbi sulla corretta abbreviazione, fare riferimento ai seguenti siti:

a. ISI Journal Abbreviations Index (http://library.caltech.edu/ reference/abbreviations/);

b. Biological Journals and Abbreviations (http://home.ncifcrf. gov/research/bja/);

c. Medline List of Journal Titles (ftp://ftp.ncbi.nih.gov/pubmed/J_Medline.txt);

iv) inserire l'anno di pubblicazione subito dopo il nome della rivista, seguito da punto e virgola;

v) NON inserire giorno o mese di pubblicazione;

vi) citare solo il volume, seguito dai due punti (NON citare il fascicolo tra parentesi);

vii) abbreviare le pagine, e.g. 351-8.

Per accertarsi di aver correttamente formattato le voci bibliografiche, confrontarle con le citazioni in PubMed (http://www.ncbi. nlm.nih.gov/pubmed).

Esempi (prestare attenzione anche alla punteggiatura):

Articolo standard su Rivista

Halpern SD, Ubel PA, Caplan AL. Solid-organ transplantation in HIV-infected patients. N Engl J Med 2002;347:284-7.

\section{Proceedings}

Christensen S, Oppacher F. An analysis of Koza's computational effort statistic for genetic programming. In: Foster JA, Lutton E, Miller J, Ryan C, Tettamanzi AG, eds. Genetic programming. EuroGP 2002: Proceedings of the 5th European Conference on Genetic Programming, 2002 Apr 3-5, Kinsdale, Ireland. Berlin: Springer; 2002. pp 182-91.

Articoli i cui autori sono Organizzazioni

Diabetes Prevention Program Research Group. Hypertension, insulin, and proinsulin in participants with impaired glucose tolerance. Hypertension 2002;40:679-86.

\section{Libri}

Murray PR, Rosenthal KS, Kobayashi GS, Pfaller MA. Medical microbiology. 4th ed. St. Louis, MO: Mosby; 2002. (CITAZIONE DEL LIBRO INTERO)

Meltzer PS, Kallioniemi A, Trent JM. Chromosome alterations in human solid tumors. In: Vogelstein B, Kinzler KW, eds. The genetic basis of human cancer. New York, NY: McGraw-Hill; 2002. pp 93113. (CITAZIONE DI UN CAPITOLO)

\section{MODALITÀ D'INVIO DEI LAVORI \\ Monografie}

Gli Autori dovranno fare riferimento alla Dr.ssa Gnerre (e-mail: pgnerre@yahoo.it) e agli eventuali altri referenti da lei designati, nelle prime fasi di stesura dei manoscritti, revisioni e correzioni. 


\section{QUADERNI}

\section{dell'Italian Journal \\ of Medicine}

La Dr.ssa Gnerre raccoglierà poi le versioni definitive dei lavori di ciascuna monografia e provvederà all'invio di tutti i materiali all'ufficio editoriale.

I lavori solo nella loro versione definitiva e approvata dalla Commissione FADOI Giovani dovranno pervenire all'ufficio editoriale già pronti per l'impaginazione e immediata pubblicazione (già corredati da eventuali permessi per la riproduzione di tabelle e immagini).

\section{Traduzioni}

Previo invito dell'Editor-in-Chief, gli Autori dovranno far pervenire all'ufficio editoriale la versione tradotta in italiano, al seguente indirizzo e-mail: paola.granata@pagepress.org

Il file in formato Word dovrà essere formattato secondo gli standard editoriali della rivista ufficiale ed essere già pronto per impagina- zione e immediata pubblicazione (corredato da eventuali permessi per la riproduzione di tabelle e immagini).

Si prega di inviare le eventuali tabelle in formato editabile e le figure in alta definizione secondo gli standard sopra riportati.

\section{NOTA PER GLI AUTORI}

I lavori pubblicati sui Quaderni ITJM non verranno indicizzati, ma saranno liberamente disponibili in un'apposita sezione del sito FADOI (http://www.fadoi.org/).

Gli Autori i cui lavori siano accettati per la pubblicazione sui Quaderni ITJM $e$ che fossero interessati a vederli pubblicati anche sulla rivista ufficiale, dovranno sottomettere attraverso il sito dell'ITJM (www.italjmed.org) la versione (già tradotta) in inglese e redatta in modo conforme alle linee guida della rivista; seguiranno poi la procedura di selezione tramite peer review e, se accettati, saranno inseriti nel piano editoriale standard.

\section{STAFF EDITORIALE}

Paola Granata, Journal Manager

paola.granata@pagepress.org

Selvaggia Stefanelli, Marketing Manager

marketing@pagepress.org

Claudia Castellano, Production Editor

Tiziano Taccini, Technical Support

\section{QUADERNI - ITALIAN JOURNAL OF MEDICINE}

Tutti gli articoli pubblicati sui QUADERNI - Italian Journal of Medicine sono redatti sotto la responsabilità degli Autori. La pubblicazione o la ristampa degli articoli della rivista deve essere autorizzata per iscritto dall'editore. Ai sensi dell'art. 13 del D.Lgs 196/03, i dati di tutti i lettori saranno trattati sia manualmente, sia con strumenti informatici e saranno utilizzati per l'invio di questa e di altre pubblicazioni e di materiale informativo e promozionale. Le modalità di trattamento saranno conformi a quanto previsto dall'art. 11 del D.Lgs 196/03. I dati potranno essere comunicati a soggetti con i quali PAGEPress intrattiene rapporti contrattuali necessari per l'invio delle copie della rivista. Il titolare del trattamento dei dati è PAGEPress Srl, via Belli 7 - 27100 Pavia, al quale il lettore si potrà rivolgere per chiedere l'aggiornamento, l'integrazione, la cancellazione e ogni altra operazione di cui all'art. 7 del D.Lgs 196/03.
PUBBLICATO DA

PAGEPress Publications

via G. Belli 7

27100 Pavia, Italy

T. +39.0382 .1751762$

F: +39.0382 .1750481$

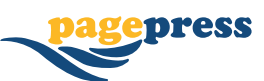

www.pagepress.org

info@pagepress.org
Pubblicato: Giugno 2014. 